\title{
th. 1829
}

ERDA/JPL/954331-77/4

SILICON MATERIALS TASK OF THE LOW COST

SOLAR ARRAY PROJECT (PHASE II)

Eighth Quarterly Report, July 1-September 30, 1977

By

R. H. Hopkins

P. D. Blais

J. R. Davis

M. H. Hanes

P. Rai-Choudhury

A. Rohatgi

J. R. McCormick

December 1977

(TIC Issuance Date)

Work Performed Under Contract No. NAS-7-100-954331

Westinghouse Electric Corporation

Research and Development Center

Pittsburgh, Pennsylvania

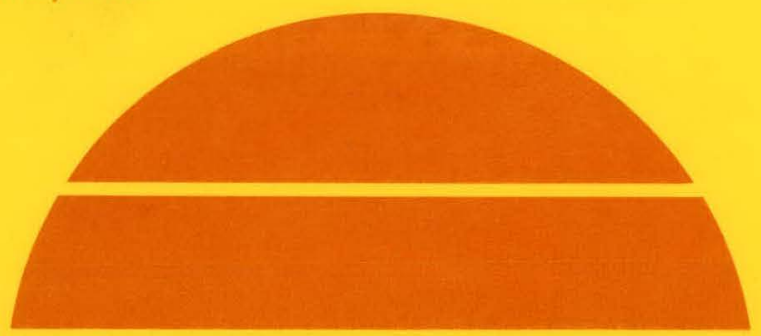

\section{MASTER}

\section{U.S. Department of Energy}

DISTRIBUTION OF THIS DOCIIMAENT IS I INI IMITED 


\section{DISCLAIMER}

This report was prepared as an account of work sponsored by an agency of the United States Government. Neither the United States Government nor any agency Thereof, nor any of their employees, makes any warranty, express or implied, or assumes any legal liability or responsibility for the accuracy, completeness, or usefulness of any information, apparatus, product, or process disclosed, or represents that its use would not infringe privately owned rights. Reference herein to any specific commercial product, process, or service by trade name, trademark, manufacturer, or otherwise does not necessarily constitute or imply its endorsement, recommendation, or favoring by the United States Government or any agency thereof. The views and opinions of authors expressed herein do not necessarily state or reflect those of the United States Government or any agency thereof. 


\section{DISCLAIMER}

Portions of this document may be illegible in electronic image products. Images are produced from the best available original document. 


\section{NOTICE}

This report was prepared as an account of work eponsored by the Uniter States Government. Neither the United States nor the United States Department of Energy, nor any of their employees, nor any of their contractors, subcontractors, or their employees, makes any w/arranty, express $\mathrm{nr}$ implied, or assumes any legal liability or responsibility for the accuracy, completeness or usefulness of any information, apparatus, product or process disclosed, or represents that its use would not infringe privately owned rights.

This report has been reproduced directly from the best available copy.

Available from the National Technical Information Service, U. S. Department of Commerce, Springfield, Virginia 22161.

Price: Paper Copy $\$ 6.00$

Microfiche $\$ 3.00$ 
ERDA/JPL/954331-77/4

Distribution Category UC-63

SILICON MATERIALS TASK OF THE LOW COST SOLAR ARRAY PROJECT (PHASE II)

Eighth Quarter1y Report

1 July 77 - 30 September 77

R. H. Hopkins, P. D. Blais, J. R. Davis, M. H. Hanes, P. Rai-Choudhury and A. Rohatgi Westinghouse Research \& Development Center and

J. R. McCormick

Dow Corning Corporation

Contract No. 954331

This work was performed for the Jet Propulsion Laboratory, California Institute of Technology under NASA Contract NAS7-100 for the U. S. Energy Research and Development Administration, Division of Solar Energy.

The JPL Low-Cost Silicon Solar Array Project is funded by ERDA and forms part of the ERDA Photovoltaic Conversion Program to initiate a major effort toward the development of low-cost solar arrays. 


\section{TECHNICAL CONTENT STATEMENT}

This report contains information prepared by the westinghouse Research and Development Center under Jet Propulsion Laboratory subcontract. Its content is not necessarily endorsed by the . Tet Propulsion Laboratory, the California Institute of Technology, or the National Aeronautics and Snace Administration.

NEW TECHNOLOCY

No new technology is renortable for the neriod covered by this report. 


\section{SUMMARY}

The objective of Phase II of this program is to investigate the effects of various processes, metal contaminants, and contaminantprocess interactions on the performance of terrestrial silicon solar cells so that purity requirements for a solar grade silicon can be delineated.

Briefly, the program approach consists in (1) the growth of הnubly and multiply-doped silicon single crystals containing a baseline boron or phosphorus dopant and specific impurities which produce deep levels in the forbidden band gap, (2) assessment of these crystals by a battery of chemical, microstructural, electrical and solar cell tests, (3) correlation of the impurity kind and level with crystal qualicy and device performance, (4) delineation of the role of impurities and processing on subsequent silicon solar cell performance, and (5) determination of the combined effects of impurities and growth rate on the crystal quality and cell performance of silicon produced by both the dendritic web and Czochralski methods. The central thrust of our activities this quarter was in three areas: the crystal growth of impurity-bearing ingots with different base doping types and concentrations; evaluation of the solar cell performance of $n$-base and p-base devices; and refinement of techniques for the analysis of solar cells subjected to various contaminants and process variations.

Our comparative study of $\mathrm{n}$ - and $\mathrm{p}$-base solar cells doped with metal impurities continues to reveal significant differences in the response of these two types of devices to contaminents. The $\mathrm{P}^{+} \mathrm{N}$ devices containing vanadium show substantially better performanre than $\mathrm{N}^{+} \mathrm{P}$ cells doped to the same metal concentration. For example $\tilde{n}$-base devices about $70 \%$ as efficient as the uncontaminated base devices ( $n=10 \%$ without AR coating) are readily produced while comparable p-base devices are only about $40 \%$ as efficient as the baseline cells. The performance improvement for the vanadium bearing $\mathrm{P}^{+} \mathrm{N}$ cells parallels that achieved previously for titanium. For nickel doping the situation is reversed. 
$\mathrm{N}^{+} \mathrm{P}$ cells with $4 \times 10^{15} \cdot \mathrm{cm}^{-3}$ nickel show little degradation compared to the baseline. However $\mathrm{P}^{+} \mathrm{N}$ cells containing similar nickel levels are only $54 \%$ as efficient as the baseline devices. The performance degradation in the $n$-base cells seems to be linked to precipitation effects in the junction rather than base lifetime reductions. Doped with iron $\mathfrak{n}$ and $p$-base devices perform in a similar fashion, the $\mathrm{P}^{+} \mathrm{N}$ cells being somewhat less efficient.

The first solar cell results for molybdenum-doped p-base material are now available. At an estimated $6 \times 10^{14} \cdot \mathrm{cm}^{-3}$ concentrations molybdenum, a material often used in. furnaces and reactors, depreciates cell performance to about $75 \%$ of the baseline efficiency. Although the chemical analysis must be refined, this result suggests that the potential effects of molybdenum be kept in mind when it is employed as a material of construction. Phosphorus compensation may be required for some forms of solar grade material; phosphorus may also be present as a contaminant in silicon. As part of this study phosphorus compensation of $4 \Omega \mathrm{cm}$ baseline material is being evaluated. For an ingot compensaten to about $15 \%$ of the boron primary doping level $\left(6 \times 10^{14} \cdot \mathrm{cm}^{-3}\right)$ phosohorous has little or no effect on cell performance.

During our previous modeling studies we observed that cells containing the impurities copper, nickel; and iron showed a reduction in performance not wholly attributable to a reduction in base lifetime. We have recently developed and tested a method for the quantitative analysis of solar cell I-V data which permits the effects of impurities on junction, base and contact behavior to be separated and accessed. This tool along with precision lifetime measurements will soon be applied to study processing and impurity phenomenon in solar cells. 


\section{INTRODUCTION}

The overall objcctive of this program is co delermine how various processes, impurities and impurity-process interactions affect the performance of terrestrial silicon solar cells. The development of such data permit the definition of the tolerable impurity levels in a low-cost Solar Grade silicon, and also identify what processes mitigate or enhance the effects of impurities in silirnn. This information can subsequently be employed in carrying out cost-tradeoff analyses for the application of Solar Grade material.

Specifically under study in Phase II of the program are:

(1) The effect of heat treatment and gettering on the properties of silicon containing deep level impurities like $\mathrm{Ti}$ and $\mathrm{Cr}$.

(2) The combined effects of metal contaninants and low base resistivity on solar cell performance.

(3) The relative performance of $p$-base and n-base solar cells containing similar levels of metal impurities.

(4) The role of multiple contaminants on solar cell behavior.

(5) The effect of grain boundaries on metal-contaminated solar cells.

(6) The interaction of oxygen, carbon, and metal impurities in solar cells.

(7) Improved techniques for the analysis of impurity effects in silicon and silicon solar cells.

Work this quarter centers primarily in areas (3), (4), and (7). In particular, we present an extended discussion of a refined approach for analyzing solar cell I-V data to quantitatively assess the role of processing and impurities on both the function and base regions of the device. Inttial experimental results in areas (1) and (5) are also described. 


\section{TECHNICAL RESULTS}

\subsection{Silicon Growth and Analysis}

The concentration ranges of the impurities ${ }^{*}$ under study are illustrated in Table 1 . The column headings denote the various substrate doping levels and base types. The choice of $0.2 \mathrm{ohm}-\mathrm{cm}$, p-type material for investigation was made after evaluating the relative performance of cells made on material with resistivity ranging from $0.5 \mathrm{ohm}-\mathrm{cm}$ through $4.0 \mathrm{ohm}-\mathrm{cm}^{3}$. In addition, a total of 17 multiply doped 4 ohm-cm ingots containing two or more of the impurities shown in Table 1 have been prepared. The resistivity of the n-base material was chosen to give comparable carrier concentration to that found in the $4 \mathrm{ohm}-\mathrm{cm}, \mathrm{p}$-base silicon.

We reiterate that the upper limit for impurities shown in Table 1 has been established by one of the following criteria: (1) the solid solubility limit, (2) the maximum concentration achievable without producing constitutional supercooling of the melt, or (3) a concentration which degrades solar cell performance beyond any potential utility. The lower limit, where established, is determined as that concentration which does not degrade cell performance. Continued analysis of Ingots enhances tile data base upon which the impurity concentrations are founded so an updated summary of the best estimate of impurity concentration and actual measured values for all ingots produced during Phase I and Phase II of this program appears in the Appendix.

\footnotetext{
${ }^{*}$ The selection of impurities for investigation is based on those elements present in metallurgical-grade silicon and quartzite or the potential impurities introduced during processing. 1
} 
Preparation/characterization of first generation doubly doped n-type ingots is now complete and preparation of low resistivity $(0.2 \mathrm{ohm}-\mathrm{cm})$ p-type ingots is also nearly done. Investigation of compensated material was initiated this quarter with ingots doped with phosphorus and boron as well as phosphorus and aluminum. Overall a total of thirteen ingots were grown; the results of the ingot preparation and characterization are presented in the following sections.

\subsubsection{Ingot Preparation}

All ingoto given Llıls quareer were prepared by the Czochralski growth method as described previously. ${ }^{1}$ The effective segregation coefficients used to determine appropriate melt concentrations are shown in Table 2. A review of our data for the impurities horon and copper indicate that the effective segregation coefficients should be 0.8 and $8 \times 10^{-4}$ respectively. These values differ slightly from those reported previously. The breakdown of ingots prepared this quarter is as follows:

5 n-type doubly-doped $(1.5 \mathrm{nhm-cm}):$ n-base studles

3 p-type doubly-doped: 4 ohm-sm matrix extenslun

2 p-type phospliurus compensated ( $4 \mathrm{ohm}-\mathrm{cm})$ : matrix extension

3 p-type single doped $(0.2 \mathrm{ohm}-\mathrm{cm})$ : low resistivity studies

Both compensated n-type and p-type ingots were grown. Aluminum was used as the compensating impurity in Ingot $W-084$ with a phosphorus to aluminum ratio of approximately 1.4 Two p-type ingots were compensated with phosphorus. The boron/phosphorus ratio of $\mathrm{W}-080$ was 6.6 while the ratio of $W-092$ was 1.13 . The buron concentration in Ingot $W-092$ is ten times that of $W-080$. 
Table 1. Impurity Matrix Under Investigation

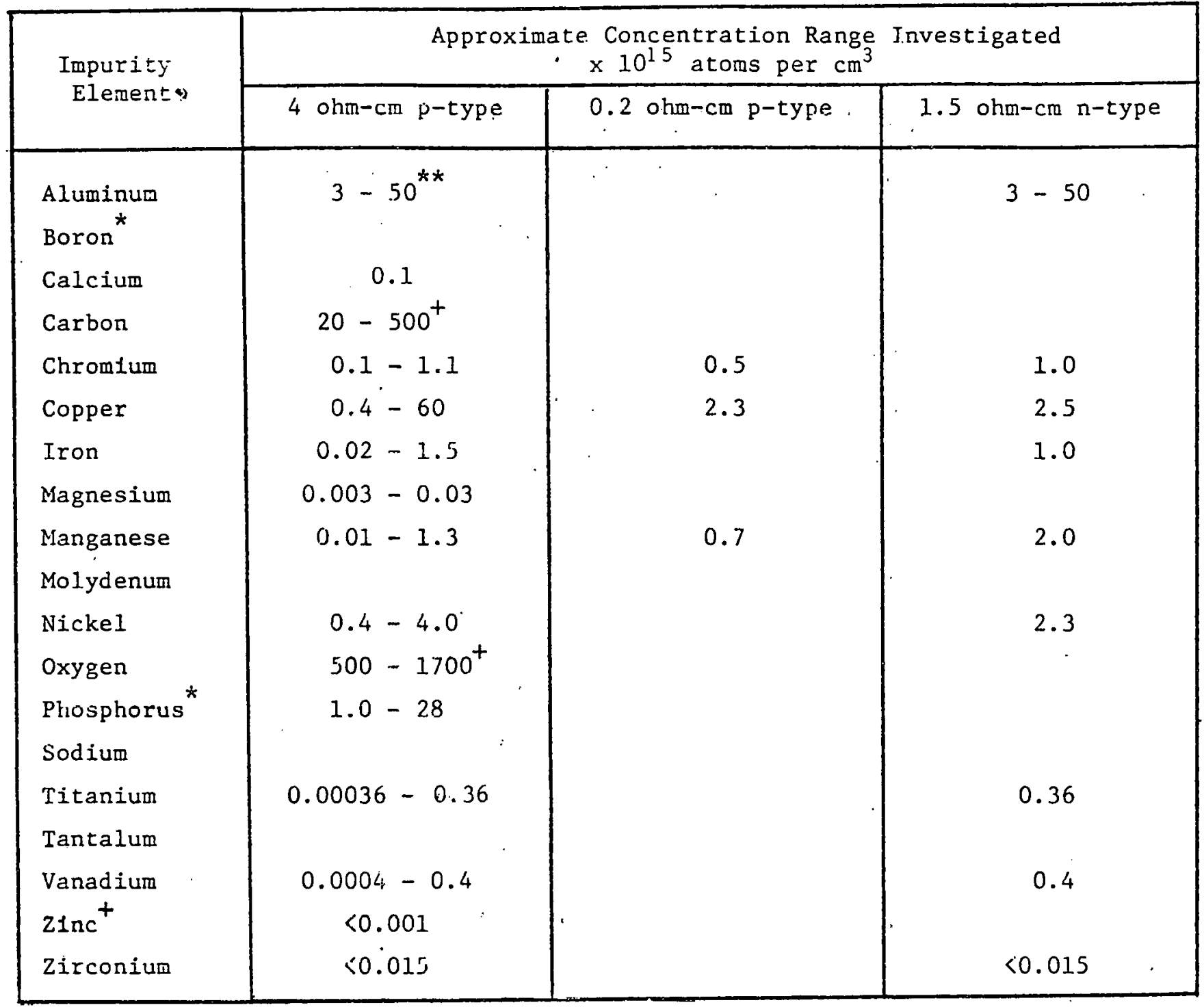

* Boron and phosphorus are electrically active impurities and therefore cause variations in resistivity when used as a secondary impurity.

$\therefore *$ Uncertainty in exact range due to discrepancy between electrical and SSMS measurements. 1

+ oxygen and carbon concentrations measured in 50 ingots doped with additional impurities. No effort yet to correlate effects if any. 
Table 2. Segregation Coefficients

\begin{tabular}{|c|c|}
\hline Element & Segregation Coefficient \\
\hline Al & $3 \times 10^{-2}\left(2.8 \times 10^{-3}\right)$ \\
\hline B & 0.8 \\
\hline C & 0.05 \\
\hline $\mathrm{Ca}$ & $?$ \\
\hline $\mathrm{Cu}$ & $8.0 \times 10^{-4}$ \\
\hline $\mathrm{Cr}$ & $1.1 \times 10^{-5}$ \\
\hline $\mathrm{Fe}$ & $6.4 \times 10^{-6}$ \\
\hline $\mathrm{Mg}$ & $3.2 \times 10^{-6}$ \\
\hline $\mathrm{Mn}$. & $1.3 \times 10^{-5}$ \\
\hline Mo & $\sim 10^{-6}$ \\
\hline $\mathrm{Ni}$ & 3. $2 \times 10^{-5}$ \\
\hline $\mathrm{Ph}$ & 0.35 \\
\hline $\mathrm{Ta}$ & $\sim 10^{-7}$ \\
\hline $\mathrm{Ti}$ & $3.6 \times 10^{-6}$ \\
\hline V & $4 \times 10^{-6}$ \\
\hline $\mathrm{Zn}$ & $10^{-5}$ \\
\hline $\mathrm{Zr}$ & $<1.5 \times 10^{-7}$ \\
\hline
\end{tabular}


Calcium metal was used successfully as a dopant for p-type, 4 ohm-cm material in Ingot $\mathrm{W}-087$. We note, however, that considerable calcium is evolved from the melt and oxide precinitates form on the melt surface early in the growth cycle which limits single crystal yield. Melt analysis indicates that approximately 90 percent of the calcium is lost from the melt during the growth period.

The yield of single crystal material was also reduced when attempts were made to achieve a high carbon concentrations. Toss of structure consistently occurs at the $4 \times 10^{17}$ to $5 \times 10^{17}$ atoms $/ \mathrm{cm}^{3}$ concentration. The latter value was achieved in the tang end of Ingot W-086. however, a twin developed prior to achieving this concentration.

As we have reported previously, it is difficult to achieve chromium concentrations of $1 \times 10^{15}$ atoms $/ \mathrm{cm}^{3}$ or greater without precipitation in the melt problems. Due to these growth problems and associated low single crystal yields, the highest $\mathrm{Cr}$ concentration achieved in $0.2 \mathrm{ohm} \mathrm{cm}$, p-type first generation material was $0.5 \times 10^{15}$ atoms $/ \mathrm{cm}^{3}$.

\subsubsection{Ingot Evaluation}

The ingot evaluation process has been discussed previously, e.g. Ref 5. Analytical data, with the exception of SSMS and NAA is up to date on all ingots. Mass soectrographic data is available on all but Ingots $W-085$ through $W-092$. Ingots have been selected for neutron activation andysis and analytical efforts will be initiated shortly.

Resistivity and etch pit densities measured on slices from Ingots W-054 through $W-092$ are tabulated in Table 3 . Etch pit densities are shown for both tang and seed ends of the ingots. The first value representing the EPD of the seed is most reoresentative of slices employed in cell analysis. No serious problems have been encountered in meeting resistivity target values. Resistivity of Ingot $W-084$ is high due to the intended aluminum compensation. Although the resistivity of $\mathrm{W}-092$ is in the $1.7-5.6 \mathrm{ohm}-\mathrm{cm}$ range, boron concentration in this ingot $3.2 \times 10^{16}$ atoms $/ \mathrm{cm}^{3}$ which in uncompensated material would correspond to $0.55 \mathrm{ohm}-\mathrm{cill}$. 
Table 3. Ingot Resistivity and Etch Pit Density

\section{Ingot Identification}

$W-054-00-000$

$\mathrm{W}-055-\mathrm{C}-\mathrm{-OOA}$

W-C:56-CL:-005

$W^{2}-057-\mathrm{CO}-000$

W*-058-00-000

W*-059-00-000

$\mathrm{H}-050-00-000$

$\mathrm{W}-0$ s1-Cr/Ti-001

$\mathrm{W}-062-\mathrm{N} / \mathrm{Cu}-001$

$\mathrm{W}-063-\mathrm{N} / \mathrm{Cr}-001$

$\mathrm{W}-064-\mathrm{N} / \mathrm{Gn}-001$

它

\begin{tabular}{l} 
TGT Res1stivity \\
(ohm-cm) \\
\hline $4.0(B)$ \\
$4.0(B)$ \\
$4.0(B)$ \\
$0.5(B)$ \\
$0.2(B)$ \\
$0.05(B)$ \\
$1.5(P)$ \\
$4.0(B)$ \\
$1.5(P)$ \\
$1.5(P)$ \\
$1.5(P)$ \\
$1.5(P)$ \\
$4.0(B)$ \\
$4.0(B)$ \\
$4.0(B)$ \\
$4.0(B)$ \\
$4.0(B)$ \\
$4.0(B)$ \\
$4.0(B)$ \\
$4.0(B)$ \\
$4.0(B)$ \\
$4.0(B)$ \\
$4.0(B)$ \\
\end{tabular}

\section{Actual Resistivity}

(ohm-2m)

$$
\begin{gathered}
4.3-3.8 \\
4.1-3.7 \\
4.4-3.75 \\
0.46-0.47 \\
0.22-0.18
\end{gathered}
$$$$
0.05-0.053
$$$$
2.1-1.0
$$$$
5.0-4.0
$$$$
2.0-0.95
$$$$
2.2-1.7
$$$$
2.2-1.35
$$$$
1.9-1.7
$$$$
6.0-3.9
$$$$
5.5-5.2
$$$$
5.2-5.1
$$$$
5.8-5.0
$$$$
2.2-1.1
$$$$
4.1-3.3
$$$$
5.0-4.5
$$$$
5.0-3.8
$$$$
4.4
$$$$
4.8-5.9
$$$$
4.8-\Xi .0
$$

$$
\begin{gathered}
\begin{array}{c}
\text { Etch Pit Density } \\
\left(/ \mathrm{cm}^{2}\right)
\end{array} \\
\hline 0-4.25 \mathrm{~K} * * \\
0.5 \mathrm{~K}-25 \mathrm{~K} \\
2.5 \mathrm{~K}-10 \mathrm{~K} \\
0.5 \mathrm{~K}-1.25 \mathrm{~K} \\
0-0.2 \mathrm{~K} \\
0-2 \mathrm{~K} \\
0-1 \mathrm{~K}
\end{gathered}
$$

$3 \mathrm{~K}$ - Clusters

$0.4-4 \mathrm{~K}$

$1 \mathrm{~K}-40 \mathrm{~K}$

$1 \mathrm{~K}-3 \mathrm{~K}$

$0-2 \mathrm{~K}$

$1 \mathrm{~K}-4 \mathrm{~K}$

$1 \mathrm{~K}-4 \mathrm{~K}$

$1 k-5 k$

$0.4 \mathrm{~K}$ - Gross Lineage

$0-1 \mathrm{~K}$

$1 \mathrm{~K}-4 \mathrm{~K}$

$0-2 k$

I K $-40 \mathrm{~K}$

$400-30 \mathrm{~K}$

$0-10 \mathrm{~K}$

N/A (PoIy) 
Table 3. Ingot Resistivity and. Etch Pit Density (cont.).

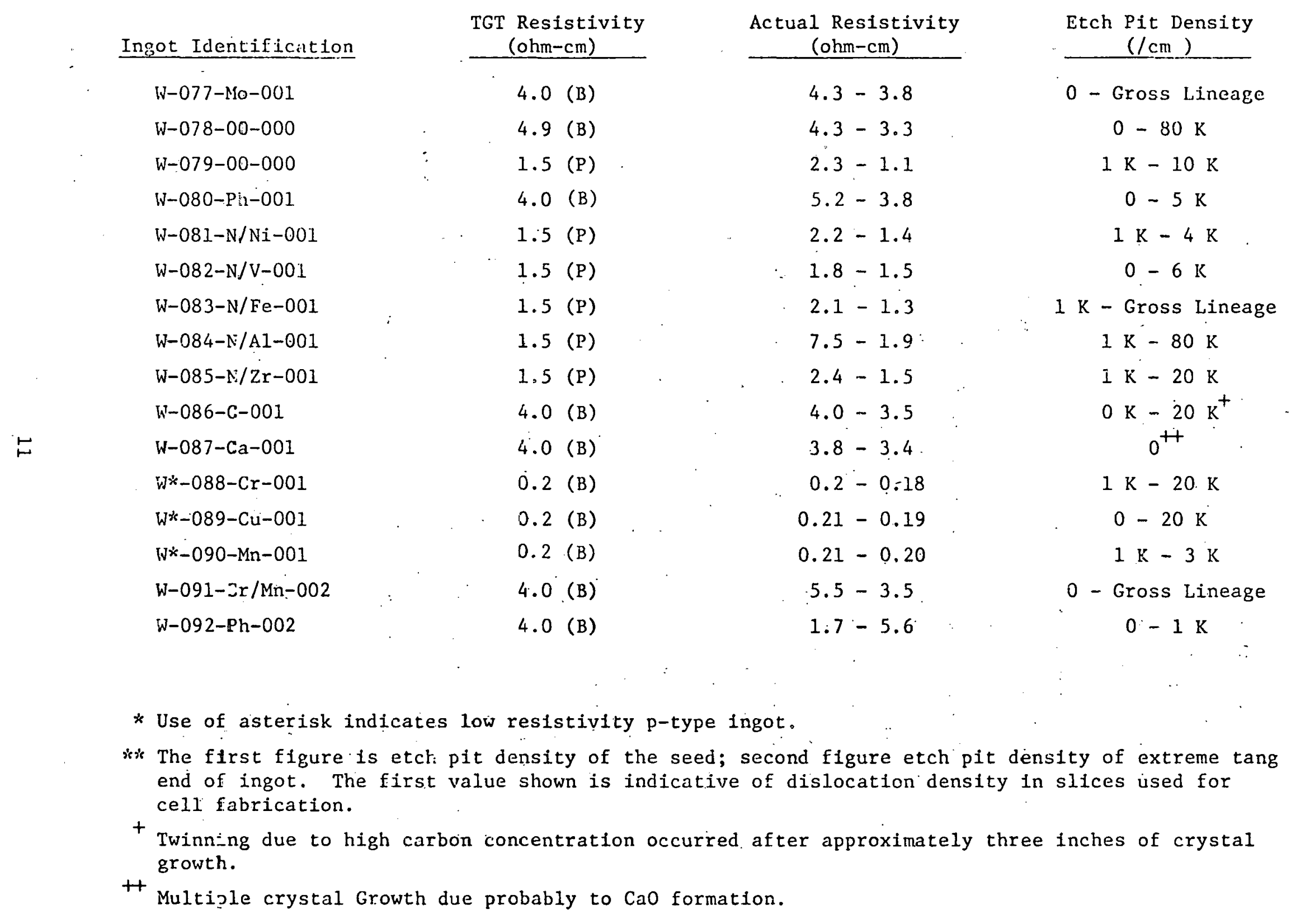


Twinning problems were encountered in the growth of ingots heavily doped with calcium, carbon, and chromium. To obtain initial data on carbon effects ingot W-086 was forwarded for solar cell fabrication/ evaluation with a twinned section extending through approximately one half the slices.

Target impurity concentrations, calculated concentrations based on melt analysis and measured impurity concentrations (SSMS or resistivity where applicable) are summarized in Table 4 for 1ngots W-054 through W-092. SSMS data is not yet available for Ingots N-085 through W-092. Resistivity measurements were employed for measuring the impurity concentration of Ingot W-092 while infrared transmission was used to determine the carbon concentration in Ingot $W-086$. It should also be noted that for some ingots shown in Table 4 target and calculated impurity concentrations have been adjusted to be consistent with the cffective segregation coefficients shown in Table 2 . In general these changes are less than 10 percent for all impurities except copper.

Aluminum concentration as determined by SSMS continues to be significantly higher than that based on resistivity measurements. This difference which has been noted by others ${ }^{2}$ has now been seen on all ingots prepared during this program. Since the aluminum doped ingors aie usually compensated with another impurity (for ingots prepared for this program) it has been impossible to establish a firm number for the ratio of total aluminum concentration to electrically active aluninum. Iron continues to pose an analytical problem due to the poor seneitivity of the SSMS to iron in silicon. Future NAA residts should provide dala on iron concentration.

Agreement ausuly target impurity concentration, calculated concentration and measured concentration continues to be relatively good. The best estimates of impurity concentration for each ingot grown during Phase IT are given in Table 5 . 
Table 4. Ingot Impurity Concentration

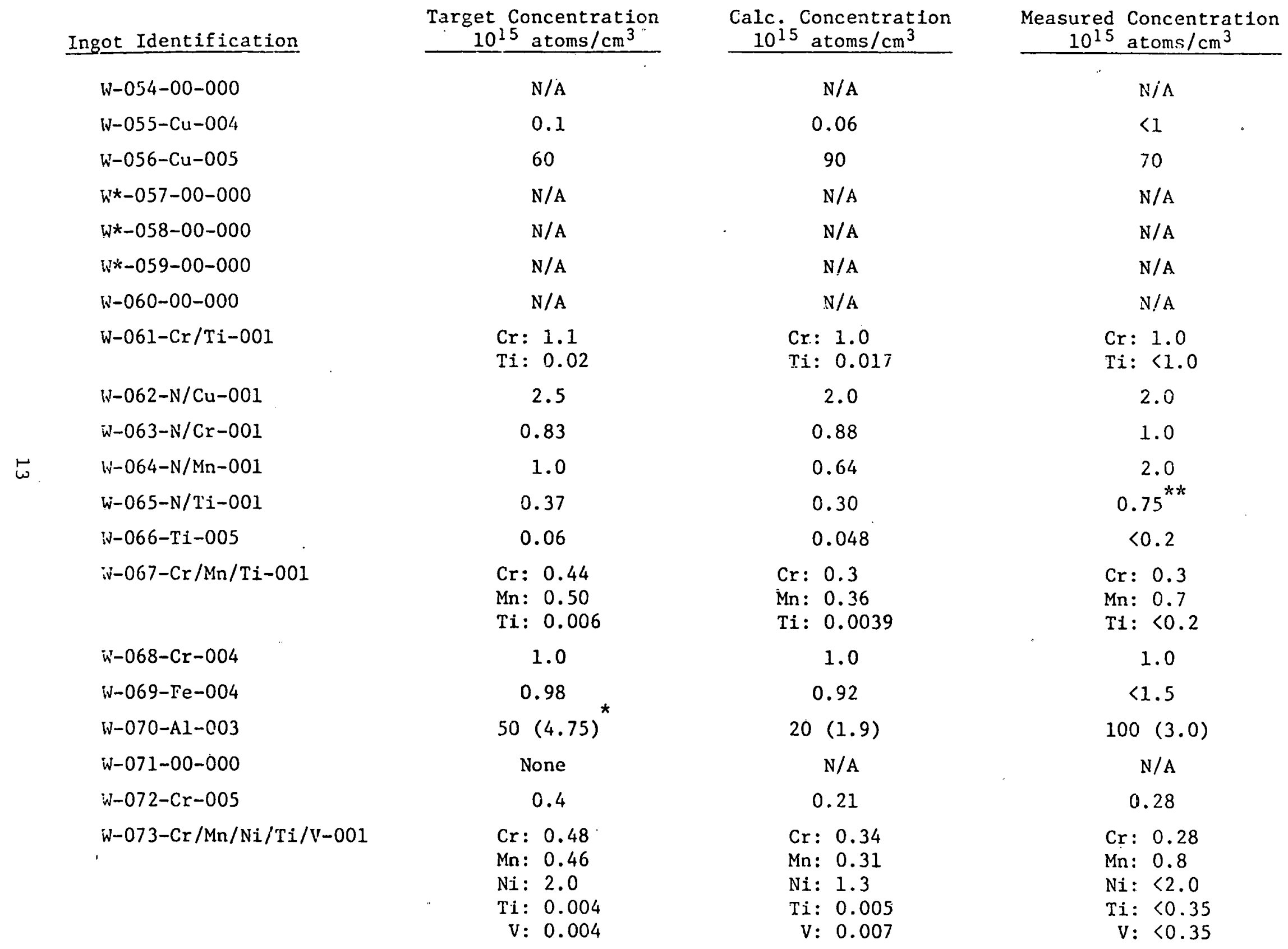


Table 4. Ingot Empurity Concentration (cont.)

\begin{tabular}{l} 
Ingot Zdentificat:- \\
\hline W-074-Cr/Mn/N1/T \\
\\
W-075-Ti/V-002 \\
W-076-Poly-002 \\
W-077-Mo-001 \\
W-078-00-000 \\
W-079-00-000 \\
W-080-Ph-001 \\
W-081-N/Ni-001 \\
W-082-N/V-001 \\
W-083-N/Fe-001 \\
W-084-N/A1-001 \\
W-085-N/Zr-001 \\
W-086-C-001 \\
W-087-Ca-001 \\
W*-088-Cr-001 \\
W*-089-Cu-001 \\
W*-090-Mn-001 \\
W-091-Cr/Mn-002 \\
W-09z-Ph-002
\end{tabular}

\begin{aligned} & $\begin{array}{r}\text { Target Concentration } \\ 10^{15} \text { atoms } / \mathrm{cm}^{3}\end{array} \\ &$\hline Cr: 0.08 \\ & $\mathrm{Mn}: 0.08 \\ & \mathrm{Ni}: 0.5 \\ & \mathrm{Ti}: 0.0006 \\ & \mathrm{~V}: 0.0006\end{aligned}$

$$
\text { T1: } 0.1
$$$$
\text { V: } 0.1
$$

None

$$
1.0
$$

None

None

0.5

2.3

0.4$$
1.0
$$$$
50(4.7)^{\star}
$$$$
0.007
$$$$
300
$$$$
1.0
$$$$
0.5
$$$$
2.3
$$$$
0 . \angle 5
$$

Cr: 0.5

$\mathrm{Mn}: 0.3$

28

\begin{tabular}{l} 
Calc \\
$10^{\text {Concentration }}$ atoms $/ \mathrm{cm}^{3}$ \\
\hline
\end{tabular}

Cr: 0.054

$\mathrm{Mn}: 0.064$

$\mathrm{Ni}: 0.28$

Ti: 0.0025

$\mathrm{V}: 0.0015$

$\mathrm{Ti}: 0.075$

V: 0.11

- N/A

0.65

$\mathrm{N} / \mathrm{A}$

N/A

0.7

0.65

0.475

0.86

22 (2.1)

0.005

N/A

0.13

0.62

2.13

0.52

0.3

0.3

$\mathrm{N} / \mathrm{A}$
Neasured Concentration $10^{15}$ atoms $/ \mathrm{cm}^{3}$

$$
\begin{array}{cl}
\text { Cr: } & <0.25 \\
\text { Mn: } & <0.25 \\
\text { Ni }:<2.0 \\
\text { Ti }:<0.25 \\
\text { V: }<0.25 \\
\text { Ti }:<0.25 \\
\text { V: }<0.25 \\
\text { N/A } \\
<0.3 \\
\text { None } \\
\text { None } \\
(0.8)^{* *} \\
<2 \\
0.85 \\
<1.5
\end{array}
$$

$40(<2.5)$

Incomp.

300-500

Incomp.

Incomp.

Incomp.

Incomp.

Incomp.

Incomp.

$(27-30) * *$

* Value in pareathesis based en resistivity measurement

t* High T1 value possibly die to vacuum leak in M.S. 
Table 5. Best Estimate of Impurity Concentration

\begin{tabular}{|c|c|c|}
\hline \multirow{2}{*}{$\frac{\text { Ingot Number }}{W-054-00-000}$} & \multicolumn{2}{|c|}{$\begin{array}{c}\text { Impurity Concentration } \\
\left(1015 \text { atoms } / \mathrm{cm}^{3}\right)\end{array}$} \\
\hline & $\mathrm{N} / \mathrm{A}$ & -- \\
\hline $\mathrm{h}-055-\mathrm{Cu}-004$ & 0.05 & $(<1)$ \\
\hline $\mathrm{W}-056-\mathrm{Cu}-005$ & 65 & $(70)$ \\
\hline$W *-057-00-000$ & $\mathrm{~N} / \mathrm{A}$ & -- \\
\hline$W^{*}-058-00-000$ & N/A & -- \\
\hline$W^{*}-059-00-000$ & $\mathrm{~N} / \mathrm{A}$ & -- \\
\hline $\mathrm{W}-060-00-000$ & $\mathrm{~N} / \mathrm{A}$ & -- \\
\hline $\mathrm{W}-061-\mathrm{Cr} / \mathrm{Ti}-001$ & $\begin{array}{ll}\text { Cr: } 1.0 \\
\text { Ti: } 0.02\end{array}$ & $\begin{array}{l}(1.0) \\
(<1)\end{array}$ \\
\hline $\mathrm{W}-062-\mathrm{N} / \mathrm{Cu}-001$ & 2.0 & $(2.0)$ \\
\hline $\mathrm{W}-063-\mathrm{N} / \mathrm{Cr}-001$ & 0.8 & $(1.0)$ \\
\hline $\mathrm{W}-064-\mathrm{N} / \mathrm{Mn}-001$ & 2.0 & $(2.0)$ \\
\hline $\mathrm{N}-065-\mathrm{N} / \mathrm{Ti}-001$ & 0.35 & $(0.75)$ \\
\hline $\mathrm{N}-066-\mathrm{T} i-00 \mathrm{~S}$ & 0.06 & $(<0.2)$ \\
\hline $\mathrm{W}-067-\mathrm{Cr} / \mathrm{Mn} / \mathrm{T} \mathrm{i}-001$ & $\begin{array}{ll}\mathrm{Cr}: & 0.4 \\
\mathrm{Mn}: & 0.5 \\
\mathrm{Ti}: & 0.006\end{array}$ & $\begin{array}{r}(0.3) \\
(0.7) \\
((0.2)\end{array}$ \\
\hline $\mathrm{W}-068-\mathrm{Cr}-004$ & 1.0 & $(1.0)$ \\
\hline $\mathrm{W}-069-\mathrm{Fe}-004$ & 1.0 & $(<1.5)$ \\
\hline$W-070-A 1-003^{k \star}$ & 50 & $(100)$ \\
\hline$W-071-00-000$ & $\mathrm{~N} / \mathrm{A}$ & - \\
\hline$W-072-\mathrm{Cr}-005$ & 0.4 & $(0.28)$ \\
\hline $\mathrm{N}-073-\mathrm{Cr} / \mathrm{Nin} / \mathrm{Ni} / \mathrm{Ti} / \mathrm{V}-001$ & $\begin{aligned} \mathrm{Cr}: & 0.4 \\
\mathrm{Mn}: & 0.5 \\
\mathrm{Ni}: & 2.0 \\
\mathrm{Ti}: & 0.004 \\
\mathrm{~V}: & 0.004\end{aligned}$ & $\begin{array}{l}(0.28) \\
(0.80) \\
(<2.0) \\
(<0.35) \\
<<0.35)\end{array}$ \\
\hline $\mathrm{H}-074-\mathrm{Cr} / \mathrm{Mn} / \mathrm{Ni} / \mathrm{Ti} / \mathrm{V}-001$ & $\begin{aligned} \text { Cr: } & 0.08 \\
\text { Mn: } & 0.08 \\
\mathrm{Ni}: & 0.5 \\
\mathrm{Ti}: & 0.0006 \\
\mathrm{~V}: & 0.0006\end{aligned}$ & $\begin{array}{l}(<0.25) \\
(<0.25) \\
(<2.0) \\
(<0.25) \\
(<0.25)\end{array}$ \\
\hline W-075-Ti/V-002 & $\begin{aligned} \mathrm{Ti}: & 0.1 \\
\mathrm{~V}: & 0.1\end{aligned}$ & $\begin{array}{l}(<0.25) \\
(<0.25)\end{array}$ \\
\hline V-076-Yoly-002 & $\mathrm{N} / \mathrm{A}$ & -- \\
\hline
\end{tabular}


Table 5. Best Estimate of Impurity Concentration (Cont.)

$$
\begin{aligned}
& \text { Ingot Number } \\
& \text { W-077-Mn-001 } \\
& \text { W-078-00-000 } \\
& \text { W-079-00-000 } \\
& \text { W-080-Ph-001 ** } \\
& \mathrm{W}-081-\mathrm{N} / \mathrm{Ni}-001 \\
& \mathrm{~W}-082-\mathrm{N} / \mathrm{V}-001 \\
& \text { W-083-N/Fe-001 } \\
& \text { W-084-N/A1-001 ** } \\
& \text { N-002-N/ } / \pi^{\circ}-001 \\
& \text { W-086-C-001 } \\
& \text { W-087-Ca-001 } \\
& W^{*}-088-\mathrm{Cr}-001 \\
& \text { W } \div \text {-089-Cu-001 } \\
& \text { W-09C-Mn-001 } \\
& \text { W-091-Cr/Mn-002 } \\
& \mathrm{W}-092-\mathrm{Ph}-002^{* *}
\end{aligned}
$$

\begin{tabular}{cc}
$\begin{array}{r}\text { Impurity } \\
\left(10^{15}\right.\end{array}$ & $\begin{array}{c}\text { Concentration } \\
\left.\text { atoms } / \mathrm{cm}^{3}\right)\end{array}$ \\
\hline 0.1 & $(<0.3)$ \\
N/A & $\cdots$ \\
N/A & - \\
0.7 & $(0.7)$ \\
1.7 & $(<2.0)$ \\
0.4 & $(0.85)$ \\
1.0 & $(<1.5)$ \\
50 & $(40)$ \\
50.015 & \\
400 & $(300-500)$ \\
$?$ & \\
0.5 & \\
2.0 & \\
0.7 & \\
$0.5 / 0.3$ & \\
28 & $(27-30)$
\end{tabular}




\subsection{Processing Studies}

The studies which form most of this task are designed to. examine the effects of both crystal growth history and the sequences of heat treatment common to solar cell processing on the performance of devices purposely contaminated with impurities. This information could then be used to develop techniques for minimizing the harmful influence of the contaminants. Much of this work has been held in abeyance pending calibration of the photoconductive decay apparatus since lifetime measurement will be employed as a major tool for assessing the way the silicon responds to the various processes. The equipment calibration is now complete (see section 3.6) and the preliminary processing studies have been initiated.

The first experiments are designed to determine the effects of high temperature cycles on recombination lifetime. Lifetime will be measured on specimens both before and after each high temperature cycle is carried out. The normalized lifetime change will be measured as a function of the maximum temperature, cooling rate from the furnace and quench temperature. Wafers $1.25 \mathrm{~mm}$ thick from ingot 078 wi11 be employed to provide baseline data for the study. The thick wafers provide for high accuracy in the PCD measurements when high lifetime material is employed. At this time specimens have been prepared and the neat treatment cycles initiated. Lifetime data on baseline and impurity-doped wafers will be reported during the next quarter.

In parallel with the heat treatment and gettering studies we are also assessing the way in which impurity doped material responds when solar cells are made with back surface fields. It has been confectured, for example, that impurities may degrade higher efficiency solar cells more severely than conventionally processed devices.

In these initial experiments wafers from ingots purposely doped with $A I$ and $C r$ were processed to solar cells by two methods: (1) our standard sequence which involves an $825^{\circ} \mathrm{POCl}_{3}$ diffusion for $50 \mathrm{~min}$. giving an $\mathrm{N}^{+} / \mathrm{P}$ device and (2) a sequence in which a back surface field 
was produced by $\mathrm{BBr}_{3}$ diffusion at $960^{\circ} \mathrm{C}$ for $20 \mathrm{~min}$. followed by front junction formation by $825^{\circ} \mathrm{POCl}_{3}$ diffusion giving an $\mathrm{N}^{+} / \mathrm{P} / \mathrm{P}^{+}$structure. The TiPdAg metallizations were made on each type of cell in the same way.

The solar cell I-V characteristics for each type of device can be compared from the data in Table 6 , which represent average values for runs of 15 to 20 cells. The raw data for the $\mathrm{N}^{+} \% \mathrm{P} / \mathrm{P}^{+}$devices appear in Tables 7 and 8 ; the data for the $\mathrm{N}^{+} / \mathrm{P}$ devices was given previous 1 y. 3 The back surface field raised the baseline (uncontaminated) cell efficiency by about $0.5 \%$. With typical Si0 AR coatings these devices would yield 14.5 to $15 \%$ efficient cells. The $\mathrm{Cr}$ containing devices remain about $77 \%$ of the baseline for both $\mathrm{N}^{+} / \mathrm{P}$ and $\mathrm{N}^{+} / \mathrm{P} / \mathrm{P}^{+}$devices. The Al containing devicices are about $72 \%$ of basellne for both the standard process cells and the back surface field devices. From these experiments we would conclude that the impurities $\mathrm{Al}$ and $\mathrm{Cr}$ tend to degrade both the standard and "high performance" devices in about the same way, so that the back surface field would provide efficiency enhancement for the contaminated material.

\subsection{N-base studies}

The comparative study of impurity effects on $p$ and $n$-base devices was continued this quarter yielding solar cell data for all but the last two first-generation ingots. The data in Table 9 illustrate how $\mathrm{V}, \mathrm{Ni}$, and $\mathrm{Fe}$ influence the performance of both device types when the impurity content of the silicon is comparable.

The most striking difference is that for $V$ : $n$-base devices doped with $4 \times 10^{14}$ at $\mathrm{cm}-3, \mathrm{~V}$ are about $70 \%$ as efficient as the baseline cells. The $p$-base devices, however, are severely degraded by $v$, being only about $40 \%$ as efficient as the baseline. The improved response of $n$-base cells to $\mathrm{V}$ parallels the improvement noted for $\mathrm{Ti}^{3}$. The data for Fe indicate a slight decrease in the $n$-base cell efficiency compared to the r-type counterpart. This is perhaps an effect of the slightly larger Fe content in the n-type ingot. The $\mathrm{Ni}$ data is perhaps the most puzzling. Here the $n$-base devices are degraded to about $55 \%$ of the baseline cells. 
TABLE 6

I-V Characteristics of $\mathrm{N}^{+} / \mathrm{P}$ and $\mathrm{N}^{+} / \mathrm{P} / \mathrm{P}^{+}$Solar Cells

Fabricated on Silicon Containing $\mathrm{Cr}$ and $\mathrm{Al}$

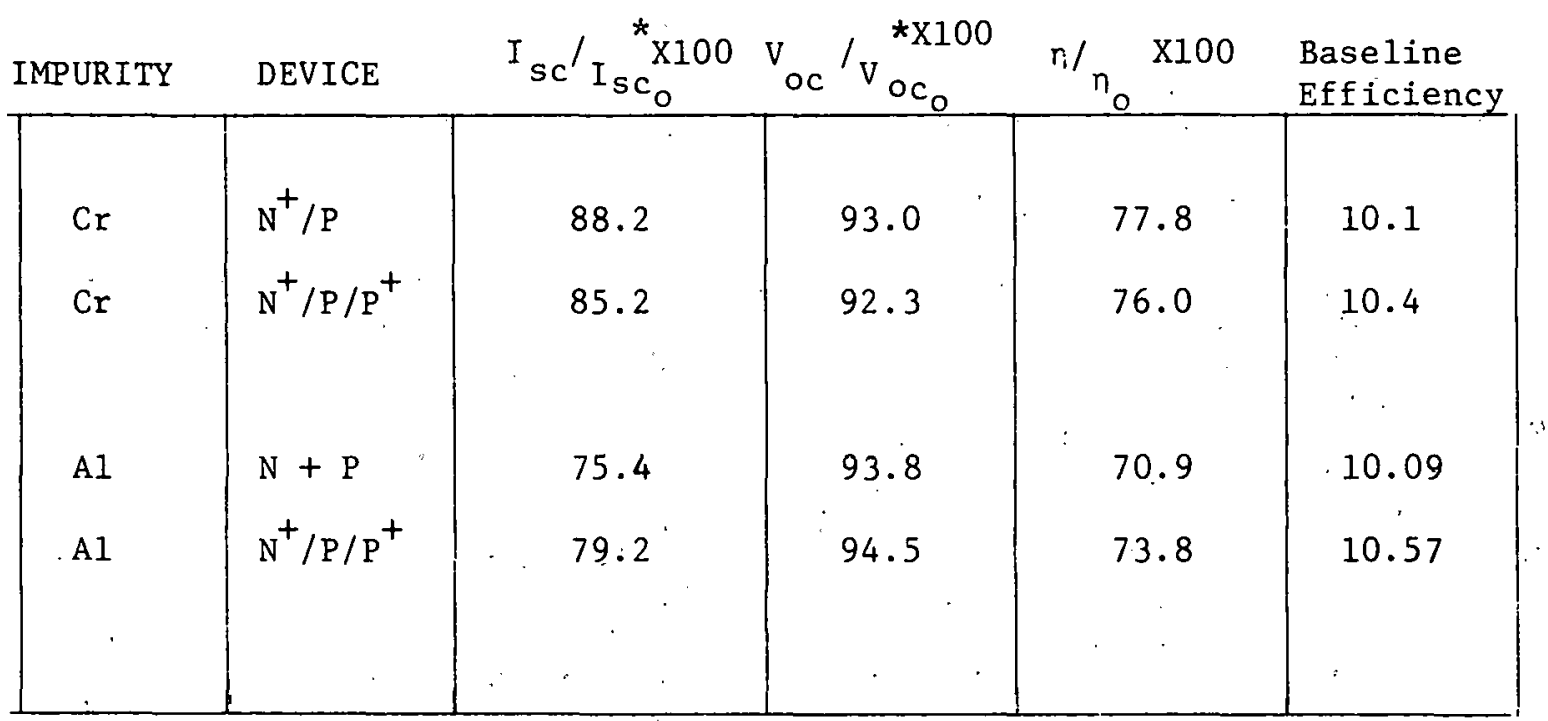


TABLE 7

$\mathrm{I}-\mathrm{V}$ Data for $\mathrm{N}^{+} / \mathrm{P} / \mathrm{P}^{+}$Cells Made on $\mathrm{Cr}$-doped Si

70708 W068CR004 (IE15) W071 00.000

SOL2 $10 / 4 / 77$ AM1: $P 0=91.60 M_{W} / \mathrm{CM}^{-2}$ NO AH COATINE

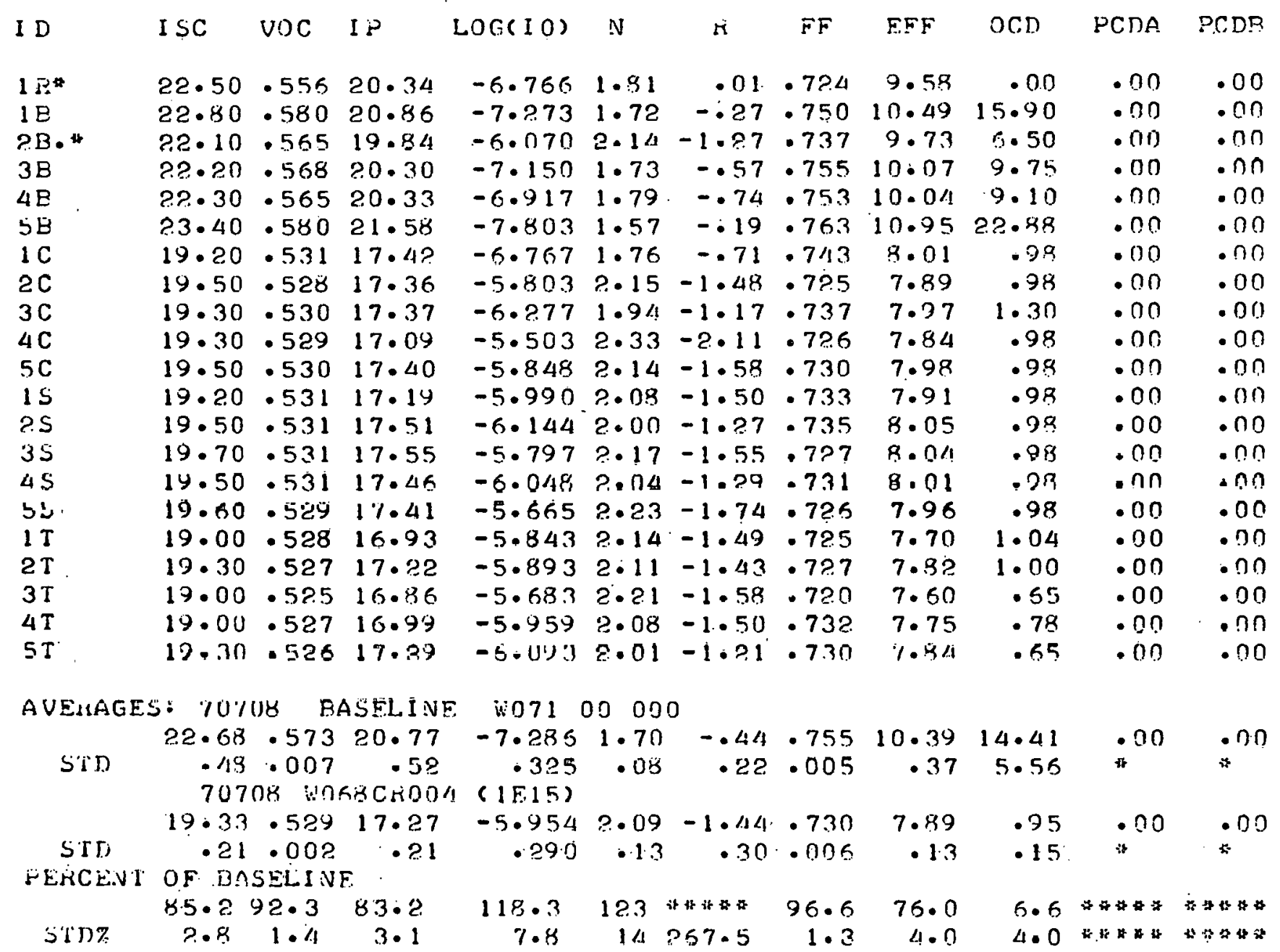


TABLE 8

$\mathrm{I}-\mathrm{V}$ Data for $\mathrm{N}^{+} / \mathrm{P} / \mathrm{P}^{+}$Cells made on Al-doped Silicon

$70507: 070 A L 003$ :05400.000

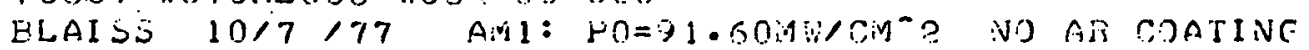

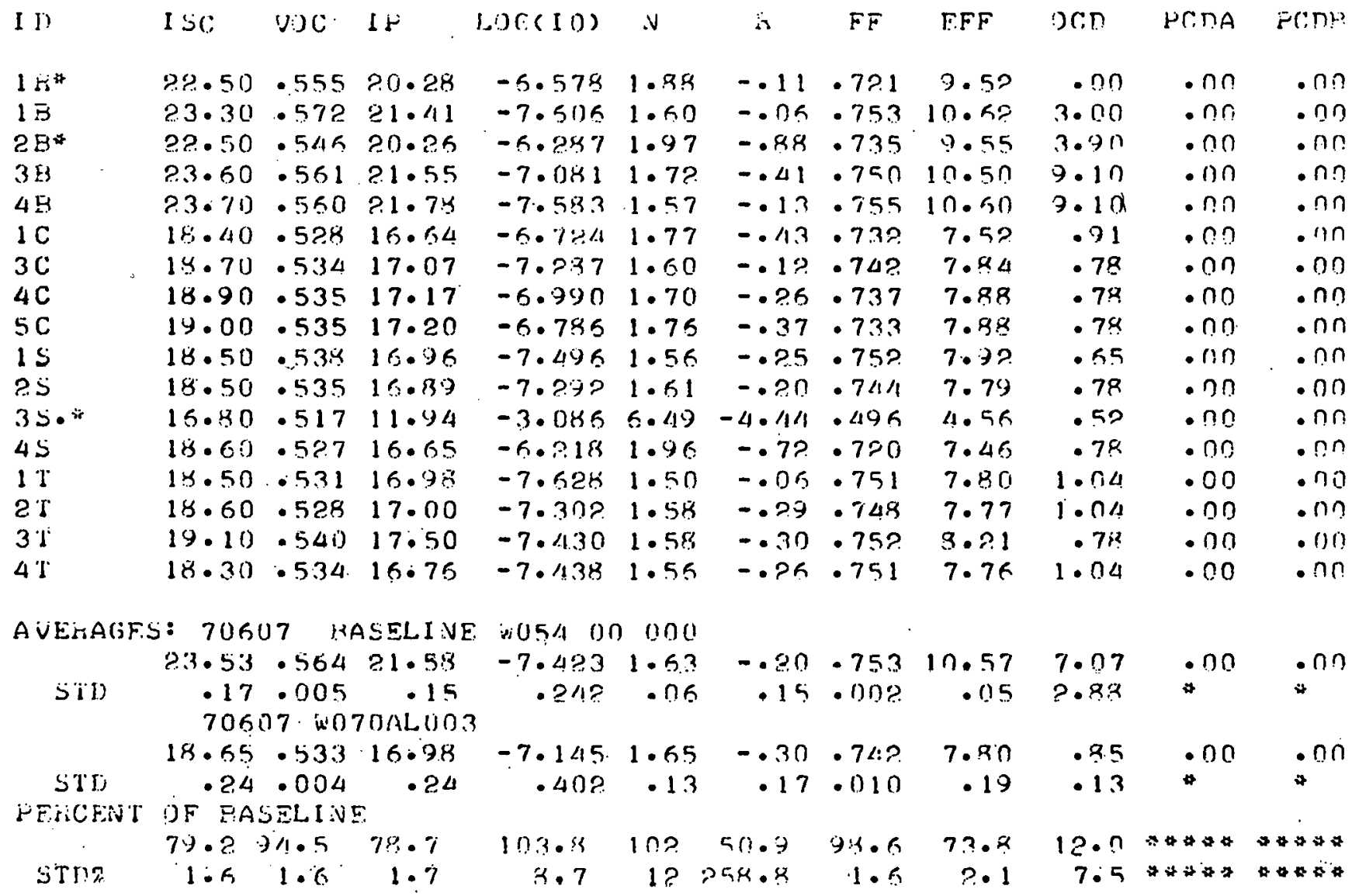




\section{TABLE 9}

Comparison of Solar Cell Performance for $n$ and p-base Devices Containing Similar Metal Contamination Levels.

\begin{tabular}{|c|c|c|c|c|c|}
\hline CELL TYPE & IMPURITY & $\begin{array}{l}\text { IMPURITY } \\
\left(1015 \text { at } \mathrm{cm}^{-3}\right)\end{array}$ & $\mathrm{c}^{*} \mathrm{Isc}_{0} \mathrm{X} 100$ & $\mathrm{c}^{*} / \operatorname{Voc}_{0} \times 100$ & $n^{*}{ }_{\mathrm{N}} \times 100$ \\
\hline $\mathbf{P}$ & V & 0.4 & 45.5 & 81.2 & 30.0 \\
\hline $\mathrm{n}$ & $\mathrm{V}$ & 0.4 & 81.2 & 91.6 & 69.8 \\
\hline $\mathrm{P}$ & $\mathrm{Ni}$ & 3.0 & 86.4 & 95.6 & 83.2 \\
\hline $\mathrm{n}$ & $\mathrm{Ni}$ & 4.0 & 98 & 74.6 & 54.8 \\
\hline$P$ & $\mathrm{Fe}$ & .85 & 89.7 & 96.3 & 86.4 \\
\hline $\mathrm{n}$ & $\mathrm{Fe}$ & 1.0 & 86.2 & 94.5 & 74.9 \\
\hline
\end{tabular}

* I-V data normalized to baseline color cells containing no added impurity. 
compared to $83 \%$ for the p-base devices. Much of the reduction in the n-base cell performance, however, is due to diminished open circuit voltage rather than short circuit current. This suggests that the junction itself rather than base lifetime is effected by Ni. Precipltation, known to occur for $\mathrm{Ni}^{1}$, may be the cause. We intend to examine this effect more closely using the quantitative I-V approach described in section 3.6 .

The raw I-V data for the $n$-base devices is presented in Tables $10-12$

\subsection{Extension of Data Base for Impurities in $4 \Omega \mathrm{cm}$ p-type Silicon.}

Three ingots were analyzed as part of this task during the past quarter: W075, a multiply-doped ingot containing $\mathrm{Ti}$ and $\mathrm{V}$, W077 doped with Mo, and w080 in which part of the boron was compensated with phosphorus. Ingot W077 was grown to broaden our information on the effects of impurities which may be used as materials of construction in reactors or furnaces. W080 is the first of a series of ingots designed to explore how compensation of the base dopant with electrically active impurities influences solar cell performance. The I-V data for the ingots are compiled in Tables 13 to 15.

The data for ingot W075, Table 13, confirms that the harmful effects of $\mathrm{Ti}$ and $\mathrm{V}$ on cell performance observed for the individual elements persists when the elements occur together. At the $10^{14} \mathrm{at} / \mathrm{cm}^{3}$ impurity level cell performance is reduced to $56 \%$ of baseline. The solar cells doped with Mo, Table 14, also show considerably reduced performance to about $74 \%$ of the baseline values, indicating this contaminant can have a significant effect when present in s111con. We estimate the impurity concentration of the ingot at about $6.5 \times 10^{14}$ $a t / \mathrm{cm}^{3}$ on the basis of the assumed segregation coefficient. The concentration is below the detection limits of SSMS so neutron activation analyses are planned for this material. The phosphorus compensation in ingot w080 is about $15 \%$ of the boron, and as the data in Table 15 indicate, at this level cell performance is not changed from the baseline values. 
TABLE 10

Solar Cell I-V Data for $n$-base Wafers Containing Hanadium.

70725 WOKCN-V001 1060 00 000

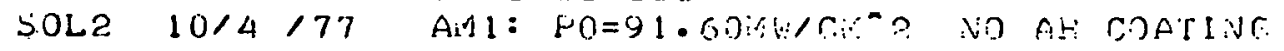

\begin{tabular}{|c|c|c|c|c|c|c|c|c|c|c|c|}
\hline 11. & 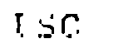 & me. & $T \dot{r}$ & I.f)( & $\mathrm{N}$ & $i i$ & $F F$ & FF & mais & HCMA & Prini \\
\hline $1 \pi^{2}$ & 2.50 & 553 & 0.21 & $6 \cdot 394$ & 1.95 & -.9 .4 & .718 & 9.45 & $\cdot 00$ & - on & - \\
\hline $1 B$ & $21 \cdot 50$ & 551 & 9.30 & 5.984 & $2 \cdot 13$ & -.98 & .723 & 9.10 & 6.50 & .00 & . $n$ \\
\hline $2 B$ & $21 \cdot 40$ & .555 & 9.69 & $-7 \cdot 78 \%$ & 1.52 & .07 & .753 & 9.45 & $6 \cdot 50$ & ח & סח • \\
\hline $3 H F R$ & 21.90 & .552 & 0.24 & -7.992 & 1.48 & -.15 & .765 & 9.97 & 9.10 & . 00 & . $\cap n$ \\
\hline $4 \mathrm{HFB}$ & 1.40 & $.55 ?$ & 19.61 & -7.453 & 1.59 & -.15 & .751 & 9.34 & 6.54 & .00 & .00 \\
\hline I CiNs & 17.20 & .511 & 15.39 & -6.33 .3 & $1 \cdot 87$ & -.33 & . $71 ?$ & $6 \cdot 6 ?$ & 1.59 & .00 & 00 \\
\hline $3 \mathrm{CNS}$ & 17.90 & .514 & 16 & $-6 \cdot 346$ & 1.87 & -.80 & - 757 & $7 \cdot 07$ & .91 & .00 & . no \\
\hline$\angle C N S$ & 17.50 & .512 & 1.5 & -5.962 & 1.64 & -.40 & .739 & $7 \cdot 0 / 1$ & .71 & .00 & .00 \\
\hline 5 Clvs & $17 \cdot 70$ & .512 & 15.95 & $-6 \cdot 542$ & $1 \cdot 79$ & -.61 & . 729 & 6.99 & $1 \cdot 56$ & - 00 & - \\
\hline 6 CNS & 17 & .510 & 15 & $-6 \cdot 2116$ & 1.90 & -.39 & .711 & $5 \cdot 75$ & 1.56 & .00 & - \\
\hline $7 \mathrm{C}$ & 17 & .512 & 15 & $-6 \cdot ?$ & 1.89 & -.86 & .7 & 5. & .91 & סח & - 0 \\
\hline 80 & 50 & .510 & 15 & -5 & $2 \cdot 18$ & $-1 \cdot 21$ & $\cdot 705$ & $6 \cdot 65$ & 1.04 & .00 & - 0 ก \\
\hline $9 \mathrm{C}$ & 70 & .510 & 15 & $-6.25 ?$ & 1.59 & -.94 & .2 & 6 & $1: 30$ & .00 & חֶ? \\
\hline 10 & 17 & $.51 ?$ & 10 & - & $1 \cdot 84$ &.- .33 & .7 & 7 & 0 & .00 & .00 \\
\hline 15 & 60 & .513 & 79 & $-6 \cdot 478$ & $1 \cdot 81$ &. .13 & $\cdot 7$ & 6.83 & $1 \cdot 30$ & .00 & .00 \\
\hline es & $17 \cdot 30$ & .513 & 01 & -0.783 & 1.73 &.$- n$ & $\cdot 7 \Omega .4$ & 0.80 & 1.04 & - $n$ & . nn \\
\hline 35 & 17 & .514 & 97 & -0.050 & 1.75 & -.35 & .720 & 0.99 & 1.04 & .00 & .00 \\
\hline 45 & 60 & .513 & 15 & -6. & 1.74 & -.28 & - 72.5 & $6 \cdot 9 ?$ & 1.69 & .00 & .00 \\
\hline $1 \mathrm{I}$ & 20 & .503 & 14 & -5 & $? .58$ & -1.66 & $\cdot 6$ & $5 \cdot 19$ & - 78 & $\cdot 1$ & .00 \\
\hline 21 & & $\cdot 508$ & 12 & -5 & 2.44 & $-1 \cdot .37$ & - & 8 & $1 \cdot 30$ & $n$ & - 0 n \\
\hline 32 & & .504 & & 1 & $? \cdot 36$ & -.41 & $\cdot e$ & $5 \cdot 15$ & - 78 & - & • \\
\hline $4 \mathrm{~T}$ & 17 & . 509 & & -6 & 1.94 & -.49 & & & 1.82 & .00 & - 0 n \\
\hline 51.7 & $17 \cdot 30$ & .508 & 9.3 & $-3 \cdot 6$ & $4 \cdot 38$ & $\cdot 56$ & $\cdot 506$ & $4 \cdot 70$ & $1 \cdot 56$ & .00 & $\cdot 00$ \\
\hline $6 T$ & $17 \cdot 50$ & .495 & 60 & -4.5144 & 3.08 & $-1 \cdot 78$ & & 5.82 & 78 & . 0 n & $\cdot 00$ \\
\hline & 07 & & $\mathrm{~N}$ & & 0 & & & & & & \\
\hline \multirow{3}{*}{ s't } & 21.57 & .556 & 19.85 & $-7 \cdot 743$ & 1.5 .3 & -.08 & & 9.60 & $7 \cdot 38$ & .00 & . 0 n \\
\hline & $\begin{array}{l}-2.4 \\
707\end{array}$ & $\begin{array}{l}.004 \\
26 \text { iv }\end{array}$ & $\begin{array}{l}B R \\
1-V 001\end{array}$ & & .05 & .11 & & & 1.?? & & \\
\hline & 17.51 & .510 & 15.56 & -6.098 & 2.02 & $\because 75$ & .710 & 6.70 & $1 \cdot ? .1$ & ח & - 0 n \\
\hline $31 \mathrm{D}$ & - 24 & .005 & -45 & - 658 & .36 & .47 & .025 & $\cdot .35$ & .34 & 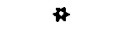 & * \\
\hline \multirow[t]{2}{*}{ EKCE.NT } & BA & SEL I & & & & & & & & & \\
\hline & $81 \cdot 2$ & $91 \cdot 5$ & $78 \cdot 4$ & 101. & 1.32 & 6 & 93.8 & $59 \cdot 3$ & 16.3 & +4 & 40 \\
\hline $10 \%$ & 2. & $1 \cdot 5$ & $3 \cdot 5$ & 21 & 2.8 & 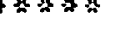 & -3 & - 7 & $8 \cdot 0$ & 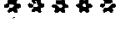 & 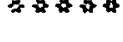 \\
\hline
\end{tabular}




\section{TABLE 11}

Solar Cell I-V Data for n-base Wafers Containing nickel.

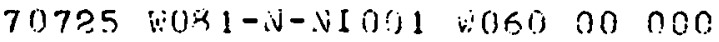

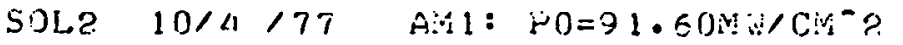

NO AIS C:JATINE

\begin{tabular}{|c|c|c|c|c|c|c|c|c|c|c|c|}
\hline $1 \mathrm{D}$ & I & vor: & $\mathrm{Ij}^{\mathrm{j}}$ & $L 1) E(I 0)$ & iN & b. & $\bar{T} \bar{F}$ & EFF & orn & Prosa & $P C \cap F$ \\
\hline $1 k *$ & $22 \cdot 50$ & 557 & 20.26 & -6.511 & $1 \cdot 9 !$ & 14 & - 7? 1 & 9.55 & . & .00 & . ח \\
\hline 13 & $21 \cdot 40$ & .557 & $19.4 ?$ & $-7 \cdot 1177$ & $1 \cdot 72$ & $\cdot 30$ & .7294 & 9.13 & 7.80 & .00 & .00 \\
\hline $2 B$ & 10 & .568 & 20.62 & -9.078 & $1 \cdot 28$ & .34 & .774 & 10.2 .7 & 9.10 & . nn & ח \\
\hline $3 B$ & $21 \cdot 50$ & .560 & 19.80 & -7.745 & $1 \cdot 19$ & - 29 & $\cdot 750$ & 9.55 & 7.80 & .00 & . $n$ n \\
\hline 48 & $21 \cdot 30$ & $.55 \dot{7}$ & 19.57 & -7.810 & $1.5 ?$ & - 3? & .745 & $9 \cdot 39$ & 6.76 & .00 & - $n n$ \\
\hline $1 \mathrm{C}$ & 21.20 & .541 & 17.89 & -4.407 & 3.31 & $-2 \cdot 34$ & .650 & 8.01 & 1.95 & .00 & . 0 n \\
\hline $\mathrm{PC}$ & $21 \cdot 10$ & .540 & $17 \cdot 5 ?$ & $-4 \cdot 140$ & 3.66 & $-2 \cdot 70$ & .644 & $7 \cdot 75$ & 1.55 & .00 & - no \\
\hline $3 \mathrm{C}$ & 2.0 .90 & .294 & 15.99 & -3.727 & 0.39 & .01 & .529 & 3.44 & 1.69 & .00 & . $n$ \\
\hline $4 C$ & 2.1 .40 & .465 & 17.018 & $-4 \cdot 656$ & 2.61 & 2.52 & .536 & 5.65 & 1.04 & . กn & .00 \\
\hline $5 c$ & $21 \cdot 30$ & .372 & 15.88 & $-4 \cdot 159$ & 2.50 & .66 & .551 & $4.6 ?$ & .55 & .00 & 0 \\
\hline 6 CiNS & 20.60 & .371 & $16 \cdot 30$ & $-4 \cdot .353$ & $2 \cdot 32$ & 1.46 & $\cdot 5.39$ & $4 \cdot 35$ & $1 \cdot 3 n$ & .00 & - nn \\
\hline $7 \mathrm{CNS}$ & 21.00 & .369 & 16.41 & -4.087 & 2.56 & .93 & .535 & 1.38 & $1 \cdot 30$ & .00 & . nn \\
\hline $8 \mathrm{CNS}$ & 20.50 & .357 & 16.28 & $-4 \cdot 140$ & 2.42 & 1.04 & .535 & 4.20 & .91 & .00 & . 0 กุ \\
\hline-9 CNS & 20.80 & .360 & 16.17 & -4.089 & 2.50 & 1.11 & .52 .3 & $4 \cdot 18$ & .91 & .00 & חס \\
\hline $10 \mathrm{CNS}$ & $21 \cdot 30$ & .414 & 17.01 & $-4 \cdot 2.98$ & 2.63 & .98 & .555 & $5 \cdot 17$ & 1.56 & .00 & .00 \\
\hline 15 & 00 & .513 & 17.04 & $-4 \cdot 2.2 .9$ & $3 \cdot 36$ & -.2? & .587 & 6.69 & 1.56 & .00 & .00 \\
\hline P.S & 50 & .447 & 61 & $-4 \cdot 309$ & 2.83 & $2 \cdot 68$ & .508 & $5 \cdot 16$ & $1.8 ?$ & .00 & . 0 n \\
\hline $3 S$ & 10 & .498 & 79 & $-4 \cdot 100$ & 3.43 & - 28 & $.56 ?$ & S.P. 5 & .65 & .00 & .00 \\
\hline $4 S$ & 30 & .436 & 61 & -4.2 .74 & 2.80 & 2.06 & - 5?? & $5 \cdot 12$ & $1 \cdot 17$ & $\cdot 00$ & .00 \\
\hline $5 s$ & 10 & .519 & 98 & -3.891 & 3.91 & $-1.9 ?$ & .598 & $6.9 ?$ & - 78 & .00 & .00 \\
\hline $1 T$ & 90 & .335 & 35 & -.3 .798 & 2.61 & -.88 & .576 & $4 \cdot 47$ & 1.95 & .00 & . \\
\hline 2.T & 2.1 .30 & .345 & 16.55 & -3.958 & 2.52 & - 54 & .536 & 1.16 & 1.82 & .00 & n \\
\hline $3 \mathrm{~T}$ & $21 \cdot 20$ & .411 & 16.85 & $-4 \cdot 401$ & 2.52 & $1 \cdot 61$ & .542 & $4 \cdot 99$ & 0.34 & .00 & - $n n$ \\
\hline $4 T$ & 21.40 & .365 & 16.54 & -4.107 & ?.Sก & $1 \cdot 2.1$ & - 5?7 7 & 4.35 & $1 \cdot 30$ & .00 & .00 \\
\hline \multicolumn{12}{|l|}{ AUEKAGES } \\
\hline \multirow{3}{*}{ STD } & $\therefore 1 \cdot 58$ & .561 & 19.85 & -7 & 1.50 & .31 & .748 & 9.58 & 7.87 & .00 & • \\
\hline & $\begin{array}{l}.31 \\
707 \%\end{array}$ & $\begin{array}{r}.004 \\
25 \quad 600\end{array}$ & $\begin{array}{c}.46 \\
-i N-N I\end{array}$ & .716 & .16 & .02 & .018 & .42 & .43 & 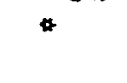 & \\
\hline & $2.1 \cdot 1 \%$ & .419 & $16 \cdot 79$ & -4.154 & 2.81 & .48 & .556 & 5. & $1 \cdot 38$ & . nn & (n! \\
\hline \multirow{3}{*}{ PERCFNT } & تجمج & .074 & .47 & - P.19 & .45 & 1.49 & .040 & $1 \cdot P 6$ & .47 & 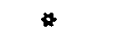 & $\pi$ \\
\hline & $B /$ & 1.8 & & & & & & & & & \\
\hline & $98 \cdot 1$ & $74 \cdot 6$ & 3 & 47.9 & 187 & 2.9 & $74 . .3$ & $54 \cdot 8$ & 17.6 & $4 \%$ & $2 \neq=1$ \\
\hline$>1 D \%$ & $2 \cdot 8$ & 13.8 & $4 \cdot 4$ & $7 \cdot 7$ & 53 & $2 \cdot 3$ & $7 \cdot ?$ & $16 \cdot 1$ & 8.5 & 2404 & 38 \\
\hline
\end{tabular}


TABLE 12

Solar Cell I-V Data for n-base Wafers Containing Iron.

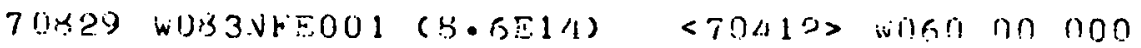

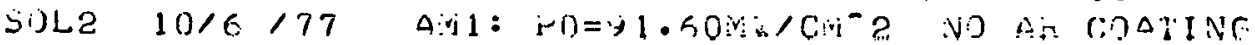

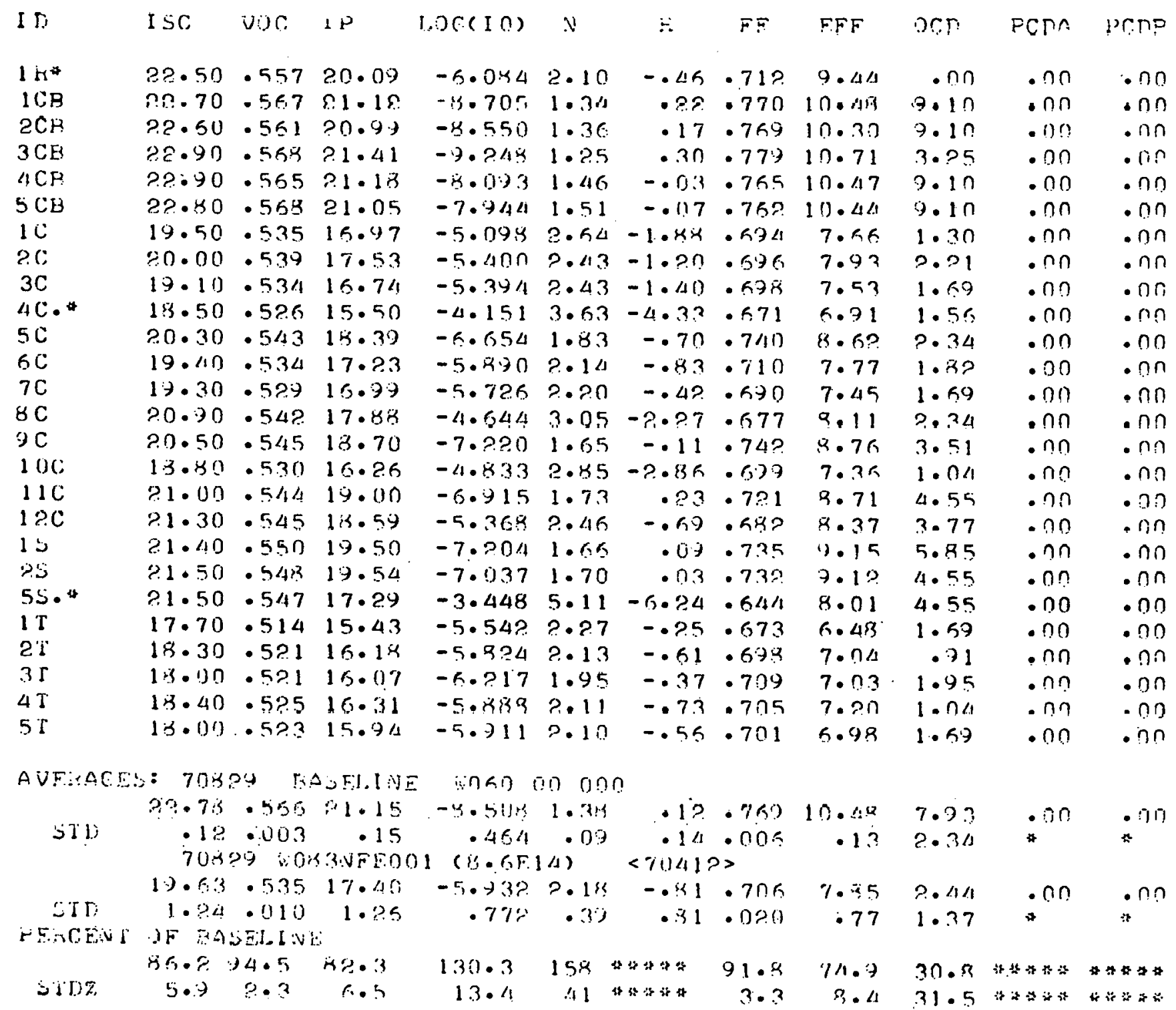


TABLE 13

Solar Cell I-V Data for $4 \Omega \mathrm{cm}$ p-type Silicon Containing $10^{14} \mathrm{at} / \mathrm{cm}^{3}$ each of Titanium and Yanadium.

$30 !=10 / 41 \%$

70614 :075TI-V002 $(1 F: 14-1 \mathrm{~F} 14) \quad$ :054 00000

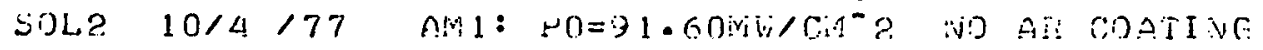

\begin{tabular}{|c|c|c|c|c|c|c|c|c|c|c|c|}
\hline $1 \mathrm{~L}$ & I $\mathrm{SC}$ & 200 & I 1 & $100(10)$ & $\mathrm{N}$ & is & FF & $T F F$ & חִ & $\because C \cap A$ & $P(\operatorname{sen}$ \\
\hline $1 \mathrm{n}^{*}$ & $2.2 \cdot 50$ & $\cdot 554$ & 20.33 & $-5 \cdot 747$ & $1 \cdot 81$ & .01 & $.7 P 3$ & 9.53 & .00 & .00 & \\
\hline $15 . *$ & $22 \cdot 20$ & .548 & 19.81 & -5.876 & $? .17=$ & $-1 \cdot 16$ & . 725 & 9.33 & 3.34 & - 110 & \\
\hline 2.8 & $? 2 \cdot 10$ & .548 & 20.03 & $-6 \cdot 6.35$ & $k=34$ & -.58 & .742 & 9.50 & 3.90 & .00 & \\
\hline $3 \mathrm{~B}$ & 20 & .549 & $20 \cdot 16$ & $-6.63 \%$ & 1.82 & -.8 .3 & - 748 & 9.65 & $3.9 n$ & .00 & \\
\hline $4 \mathrm{~B}$ & $22 \cdot 20$ & .549 & 20.42 & $-7 \cdot 620$ & 1.54 & -.27 & .750 & 9.80 & $4 \cdot 16$ & .00 & \\
\hline $1 \mathrm{C}$ & $15 \cdot 40$ & .486 & $1.3 \cdot 79$ & $-6 \cdot 604$ & 1.69 & .38 & - $70 ?$ & 5.56 & .? & .00 & 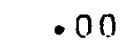 \\
\hline $2 . C$ & $15 \cdot 30$ & .485 & $13 \cdot 52$ & -6.309 & 1.30 & .0 .3 & - 699 & $5 \cdot 49$ & .13 & $\cdot \cap 0$ & 00 \\
\hline $3 C$ & 15.50 & .483 & $1.3 \cdot 73$ & -6.250 & 1.30 & -.09 & .700 & $5 \cdot 5 / 1$ & .39 & .00 & \\
\hline $4 C$ & $15 \cdot 50$ & .483 & 13.70 & -5.953 & 1.95 & -.45 & .696 & 5.51 & . 39 & $\cdot 00$ & 0 \\
\hline $5 \mathrm{C}$ & $15 \cdot 40$ & .482 & 13.55 & $-5.9 ? 3$ & 1.96 & .06 & .681 & 5.35 & .91 & . & . \\
\hline $6 C$ & $15 \cdot 40$ & .479 & 13.49 & $-5 \cdot 732$ & $2 \cdot 04$ & -.16 & .677 & $5 \cdot ? .8$ & .65 & - & \\
\hline $7 \mathrm{C}$ & $15 \cdot 40$ & .483 & $13 \cdot 66$ & -6.190 & 1.84 & .08 & .693 & $5: 45$ & .71 & . $n$ n & \\
\hline $8 \mathrm{C}$ & 15.50 & .482 & $13 \cdot 71$ & -6.178 & 1.84 & .44 & - 6834 & $5 \cdot 40$ & .91 & .00 & \\
\hline $9 \mathrm{C.*}$ & $15 \cdot 40$ & .478 & $12 \cdot 71$ & $-4 \cdot 214$ & $3.32=$ & $-3 \cdot 15$ & .634 & $\Delta \cdot 9 \Delta$ & .39 & .00 & \\
\hline $10 \mathrm{C}$ & 15.70 & .483 & 1.3 .91 & -6.176 & 1.85 & .24 & .687 & $5.5 ?$ & $\bullet 91$ & .00 & \\
\hline $11 \mathrm{C}$ & 15.60 & .480 & $13 \cdot 66$ & $-5 \cdot 72.9$ & 2.04 & -.14 & .677 & $5 \cdot 36$ & .91 & ח!ח & \\
\hline $1 \mathrm{~S}$ & $15 \cdot 70$ & .483 & 13.80 & $-5 \cdot 7 / 17$ & $2 \cdot 0.5$ & -.59 & - 689 & $5 \cdot 5.3$ & .91 & .00 & \\
\hline 25 & 15.60 & .436 & 13.91 & -6.255 & 1.83 & -.36 & $\cdot 707$ & 5.67 & .39 & - nn & \\
\hline $3 s$ & 15.90 & .486 & $14 \cdot 1 ?$ & -6.117 & 1.88 &.- .35 & $\cdot 701$ & $5 \cdot 73$ & $1 \cdot 04$ & .00 & \\
\hline 45 & $15 \cdot 70$ & .486 & $1.3 \cdot 97$ & $-5 \cdot 15 i s$ & 1.86 & -.41 & $\cdot 70.5$ & $5 \cdot 69$ & .65 & - 0 & \\
\hline $1 T$ & 15.10 & .483 & 13.51 & -6.568 & $1 \cdot 70$ & .30 & .703 & $5 \cdot 12 ?$ & .65 & -00 & \\
\hline $2 T$ & $15 \cdot 10$ & .480 & $1.3 \cdot 40$ & -6.316 & $1 \cdot 78$ & .47 & - 689 & $5 \cdot ? 8$ & .55 & .00 & \\
\hline $3 T$ & $15 \cdot 10$ & .481 & 13.51 & -6.608 & $1 \cdot 68$ & .46 & .700 & 5.38 & .65 & .00 & \\
\hline $4 \mathrm{~T}$ & $15 \cdot 10$ & .481 & $13 \cdot 22$ & -5.82 .3 & $? .01$ & . 93 & .672 & $5 \cdot 16$ & .91 & .00 & \\
\hline $5 \mathrm{~T}$ & $15 \cdot 30$ & $.48 ?$ & $13 \cdot 68$ & -6.545 & $1 \cdot 70$ &. .31 & . 70? & 5.47 & .91 & . & \\
\hline $6 T$ & $15 \cdot 30$ & .483 & $13 \cdot 68$ & -6.514 & 1.7? & .17 & $\cdot 704$ & 5.50 & $\cdot 91$ & .00 & \\
\hline cisis & 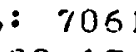 & . & $A S=$ & 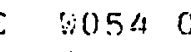 & 0000 & & & & & & \\
\hline \multirow{3}{*}{ SID } & $22 \cdot 17$ & .549 & $20 \cdot ? .1$ & -6.960 & $1 \cdot 7.3$ & -.59 & $\cdot 750$ & 9.65 & 3.99 & .00 & \\
\hline & $\begin{array}{l}.05 \\
106\end{array}$ & $\begin{array}{l}.000 \\
4 \quad 60 \%\end{array}$ & $\begin{array}{l}16 \\
1-00\end{array}$ & $\begin{array}{l}.45 .3 \\
: 1514\end{array}$ & 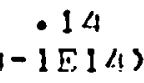 & - ?.4 & .008 & 12 & - 1? & & \\
\hline & $15 \cdot 43$ & .433 & $13 \cdot 53$ & $-6 \cdot 135$. & $1 \cdot 85$ & .0 .3 & . $594 !$ & 5.47 & . 70 & - 00 & .0 \\
\hline \multirow{3}{*}{$\begin{array}{c}\text { STD } \\
\text { PEHCF.VT }\end{array}$} & - 2ج8 & $.00 z$ & .20 & - $\sin 7$ & .12 & • & .010 & .14 & - 25 & 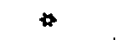 & 4 \\
\hline & OF HAS & ELISIF & & & & & & & & & \\
\hline & $\begin{array}{r}69.5 \\
1.9\end{array}$ & 8.0 & 67.7 & $111 . / 1$ & $10 \%$ & $0.5 \cdot 2$ & 92.5 & $56 \cdot 6$ & 17.6 & $\# \#$ & 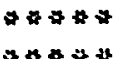 \\
\hline & $1 \cdot 2$ & $\cdot 4$ & $1 \cdot 3$ & $10 \cdot 1$ & in & & $? \cdot 3$ & ?.? & 7. & & \\
\hline
\end{tabular}


TABLE 14

Solar Cell I-V Data for $4 \Omega \mathrm{cm}$ p-type Silicon Containing Molybdenum.

70615 W07710001 (6.5E14) 054 00 000

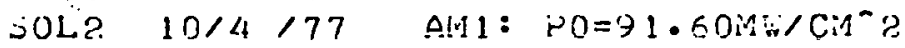
NO SK COATING

\begin{tabular}{|c|c|c|c|c|c|c|c|c|c|c|c|}
\hline $1 D$ & I SC & Vi) $C$ & Ir & $\therefore G(I 0)$ & N & 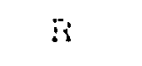 & $F F$ & FFF & DCD & prina & "r)!r: \\
\hline $1 \mathrm{~K}^{4 *}$ & • 50 & .551 & $20 \cdot 12$ & -6.149 & 2.04 & -.4 .3 & .714 & 9.37 & $.0 n$ & .00 & . on \\
\hline $1 B$ & $2.2 \cdot 70$ & .551 & $20 \cdot 79$ & 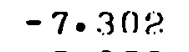 & 1.53 & - . 3? & $\cdot 75.3$ & 9.96 & 5.60 & .00 & .00 \\
\hline$P B$ & $2.2 \cdot 00$ & .548 & 20.07 & -7.072 & 1.69 & $-.4 ?$ & $.74 K$ & $9 \cdot 54$ & $4 \cdot 40$ & $\cdot n$ & - 00 \\
\hline $3 E$ & $2 ? 70$ & .550 & 20.98 & -7.956 & 1.46 & -.19 & .767 & $10 \cdot 13$ & $5 \cdot \geq 0$ &. no & . \\
\hline $4 B$ & 22.30 & - 549 & $20 \cdot 41$ & $-7 \cdot 236$ & $1 \cdot 64$ & -.45 & .755 & 9.77 & $5 \cdot ? 0$ & $.0 n$ & . 0 n \\
\hline $5 \mathrm{~B}$ & $22 \cdot 60$ & .549 & 20.76 & -7.672 & $1.5 ?$ & .06 & $\cdot 751$ & 9.85 & 4.55 & .00 & .00 \\
\hline $1 \mathrm{C}$ & $19 \cdot 10$ & .504 & $17 \cdot 07$ & -6.152 & 1.90 & -.66 & .718 & $7 \cdot 30$ & . 5? & .00 & .00 \\
\hline$P C$ & $18 \cdot 70$ & .504 & 16.74 & -6.190 & (.8) & -.73 & . 720 & $7 \cdot 18$ & $1 \cdot 04$ & .00 & - nn \\
\hline $3 \mathrm{C}$ & 18.80 & .504 & $16 \cdot 72$ & -5.886 & 2.02 & -1.01 & .715 & 7.15 & .91 & . & $.0 n$ \\
\hline $4 C$ & 18.80 & .503 & $16 \cdot 39$ & -5.2 .14 & 2.41 & $-1 \cdot 6,0$ & .694 & 6.94 & .91 & .00 & - no \\
\hline $5 C$ & 18.70 & .505 & $16 \cdot 78$ & -6.267 & 1.86 & -.82 & .726 & $7 \cdot 2.5$ & - 78 & . 00 & - nn \\
\hline 6C & 18.80 & .505 & $16.8 ?$ & $-5 \cdot 100$ & 1.9 .3 & $-1 \cdot 0 ?$ & - 725 & $7 \cdot ? 8$ & .91 & . nn & - nn \\
\hline $7 \mathrm{C}$ & $18 \cdot 60$ & .503 & $15 \cdot ? .4$ & -5.160 & 2.15 & -2.04 & - $70 ?$ & 6.95 & .91 & .00 & . \\
\hline SC & $19 \cdot .50$ & .506 & $17 \cdot 23$ & -5.672 & 2.13 & -1.01 & .706 & $7 \cdot 37$ & .91 & • nn & - \\
\hline $9 \mathrm{C}$ & $18 \cdot 70$ & .503 & 16.62 & $-5.91 ?$ & 2.01 & -.86 & .711 & $7 \cdot 08$ & .65 & . ח & - กn \\
\hline $10 \mathrm{C}$ & $18 \cdot 50$ & .504 & 16.87 & -6.317 & 1.83 & -.54 & $\cdot 72.3$ & 7.35 & .91 & $.0 n$ & .00 \\
\hline 15 & $18 \cdot 10$ & .505 & 16.51 & -6.P.?1 & 1.8 .5 & -1.01 & .780 & 7.17 & -65 & - 00 & - \\
\hline בs & 18.80 & .505 & 16.91 & -6.455 & $1 \cdot 78$ & -.52 & .725 & $7 \cdot 28$ & .78 & .00 & - חก \\
\hline $3 \mathrm{~S}$ & 19.00 & .505 & $17 \cdot 0.3$ & $-5 \cdot ? 50$ & $1 \cdot 86$ & -.70 & .72 .3 & $7 \cdot 33$ & $\cdot 65$ & . no & - 00 \\
\hline $4 \mathrm{~b}$ & 19.00 & .503 & 15.98 & -6.099 & 1.92 & -.8 .1 & - 7200 & $7 \cdot 2 x$ & - 78 & $\cdot 90$ & - $n$ \\
\hline 55 & $19 \cdot 09$ & .503 & $17 \cdot 11$ & -6.524 & 1.75 & -.114 & - 726 & $7 \cdot 34$ & .91 & • กn & .00 \\
\hline $1 \%$ & $17 \cdot 50$ & .508 & $17 \cdot 47$ & -6.179 & 1.90 & -.31 & : 72/4 & $7 \cdot 58$ & .91 & $\cdot 00$ & - 0 n \\
\hline $2 \%$ & $19 \cdot 30$ & .508 & $17 \cdot 41$ & -6.533 & $1 \cdot 76$ & -.58 & $\cdot 7.31$ & $7 \cdot 58$ & .91 & - ח & חִ \\
\hline 31 & $19 \cdot 40$ & .507 & $17 \cdot 39$ & -5.243 & 1.837 & -.71 & • 79.3 & $7 \cdot 5 ?$ & .91 & . 00 & . ก0 \\
\hline $4 \pi$ & 19.40 & .507 & 17.42 & $-6 \cdot .51$ & 1.36 & -.35 & .724 & $7 \cdot .58$ & $1 \cdot 04$ & . 0 ? & . חก \\
\hline $5 T$ & 19.30 & - 505 & $17 \cdot 35$ & -6.373 & 1.81 & -.63 & .726 & $7 \cdot 50$ & $1 \cdot n 4$ & • & - $0 n$ \\
\hline $6 T$ & $19 \cdot 40$ & .505 & $17 \cdot 30$ & -6.046 & $1 \cdot 95$ & -.72 & .715 & $7 \cdot 41$ & $1 \cdot 17$ & $\cdot 00$ & .00 \\
\hline UE & 7061 & & ASFLINE & WD540 & 00000 & & & & & & \\
\hline & $22 \cdot 45$ & .549 & 20.50 & $-7.44 R$ & $1 \cdot 59$ & -. . & .755 & 9.35 & 4.99 & $\cdot 00$ & .00 \\
\hline STD & - 27 & .001 & .32 & -3?: & .09 & .18 & $\therefore 017$ & $\cdot 17$ & .45 & $=$ & $\Delta$ \\
\hline & 19.00 & $\begin{array}{l}1.505 \\
.505\end{array}$ & 77190001 & $\begin{array}{l}6.5 .5514 \\
-6.038\end{array}$ & 1.94 & & & & & & \\
\hline$=11$ & - 32 & . 00 ? & $\begin{array}{r}10.87 \\
.35\end{array}$ & $\begin{array}{r}.357 \\
.357\end{array}$ & $\begin{array}{r}1.94 \\
-1 ; 3\end{array}$ & $\begin{array}{r}-.37 \\
.35\end{array}$ & $\begin{array}{l}\text {-7a0 } \\
. \operatorname{nny.~}\end{array}$ & $\begin{array}{r}7.30 \\
.12\end{array}$ & $\begin{array}{r}.17 \\
.15\end{array}$ & $\bullet$ & $\because 01$ \\
\hline CEDI & $J$ J & OEL I & & & & & & & & & \\
\hline & $\begin{array}{r}31.5 \\
? .5\end{array}$ & - 7 & $8 ? \cdot 1$ & 11 ये。 & $1 ?=$ & 54 & 95.4 & $74 \cdot 1$ & $17 \cdot 4$ & * & 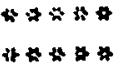 \\
\hline$=10 \%$ & $? 5$ & - 5 & $3 \cdot n$ & $3 \cdot 5$ & 17 & $57 \cdot 5$ & $2 \cdot 1$ & $3 \cdot \pi$ & - 9 & & \\
\hline
\end{tabular}




\section{TABLE 15}

Solar Cell I-V Data for 4 תcm p-type Silicon. Containing Phosphorus.

SOL? $\quad 10 / 4 / 77$

70721 WOBOPHOOL W054 $00 \quad 000$

SOL? $10 / 4 / 77$ AM1: $\quad H 0=91.60 \mathrm{MW}^{-} \mathrm{CM}^{-2}$

\begin{tabular}{|c|c|c|c|c|c|c|c|c|c|c|c|}
\hline I D & I SC & voc & I P & LOE(IO) & $N$ & 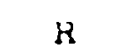 & $F F$ & $E F F$ & $O C D$ & PCDA & PCDR \\
\hline $1 R^{*}$ & $22 \cdot 50$ & .553 & $2.0 \cdot 18$ & $-6 \cdot 347$ & $1 \cdot 97$ & -.21 & .715 & 9.41 & . no & .00 & . \\
\hline $1 \mathrm{~B}$ & 1.50 & .547 & $19 \cdot 73$ & -7.456 & 1.58 & -.29 & .755 & 9.39 & 3.90 & .00 & .00 \\
\hline $2 B$ & 21.90 & . 549 & $19.4 ?$ & -6.514 & 1.89 & -.86 & .743 & 9.44 & 3.90 & .00 & . $n$ n \\
\hline $3 B$ & $21 \cdot 40$ & .546 & $19 \cdot 30$ & $-6.31 ?$ & 1.96 & $-1 \cdot 1 \cdot 1$ & .741 & 9.15 & 3.2 .5 & .00 & .00 \\
\hline $4 R$ & 21.60 & - 546 & 19.64 & -6.823 & 1.77 & -.64 & .747 & 9.31 & $3 . ? 5$ & .00 & .00 \\
\hline $1 \mathrm{C}$ & $22 \cdot 30$ & . 550 & $20 \cdot 37$ & $-7 \cdot 120$ & $1 \cdot 68$ & -.41 & .750 & $9 \cdot 73$ & $4 \cdot 55$ & .00 & .00 \\
\hline $2 \mathrm{C}$ & $22 \cdot 10$ & .549 & $20 \cdot 13$ & -6.905 & $1 \cdot 75$ & -.59 & .748 & 9.60 & $4 \cdot 94$ & .00 & .00 \\
\hline $3 \mathrm{C}$ & $22 \cdot 20$ & . 550 & $20 \cdot 15$ & -6.794 & 1.79 & -.64 & .746 & 9.64 & $5 \cdot 8.5$ & $\cdot 00$ & 00 \\
\hline $4 \mathrm{C}$ & $22 \cdot 30$ & .549 & $20 \cdot 47$ & -7.540 & 1.56 & $=.11$ & .752 & 9.74 & $5 . ? 0$ & .00 & 0 \\
\hline $5 \mathrm{C}$ & 22.10 & .546 & $20 \cdot 0.3$ & $-6 \cdot 741$ & 1.79 & -.41 & . 7.37 & $9 \cdot 40$ & 5.46 & .00 & 0 \\
\hline $6 \mathrm{C}$ & $22 \cdot 20$ & .546 & $19 \cdot 7.3$ & -5.860 & 2.17 & -.69 & .709 & 9.09 & $4 \cdot .55$ & OO' & 00 \\
\hline $7 \mathrm{C}$ & $22 \cdot 10$ & .549 & $20 \cdot 24$ & $-7 \cdot 245$ & 1.64 & -.52 & .757 & 9.72 & $5 \cdot 85$ & .00 & - \\
\hline $8 \mathrm{C}$ & $22 \cdot 00$ & . 546 & $19 \cdot 65$ & -5.9 .39 & $2 \cdot 13$ & $-1 \cdot 10$ & $.7 P .6$ & 9.2 .2 & $4 \cdot .55$ & .00 & .0 \\
\hline $9 \mathrm{C}$ & $2.2 \cdot 00$ & .545 & 19.67 & -6.545 & 1.86 & -.51 & . 732 & 7.29 & $5 \cdot ? 0$ & .00 & .00 \\
\hline 15 & $21 \cdot 70$ & .548 & 19.77 & -6.961 & 1.73 &. .53 & - 7/1R & 9.40 & $4 \cdot 55$ & .00 & .00 \\
\hline 25 & $22 \cdot 10$ & .550 & $20 \cdot 38$ & -7.973 & 1.45 & .11 & .757 & 9.72 & $6 \cdot 50$ & .00 & . \\
\hline $3 s$ & 22.00 & .548 & 20.16 & $-7 \cdot .349$ & 1.61 & -.35 & .755 & 9.62 & 5.85 & .00 & .00 \\
\hline $4 S$ & $22 \cdot 00$ & .546 & 19.90 & -6.570 & 1.86 & -.63 & - 737 & $9 \cdot 37$ & 5.20 & .00 & .00 \\
\hline 55 & $21 \cdot 90$ & . 547 & $20 \cdot 05$ & $-7 \cdot 315$ & 1.62. & -.32 & .753 & 9.53 & 5.85 & .00 & .00 \\
\hline $1 T$ & $21 \cdot 70$ & .548 & 19.87 & $-7 \cdot 440$ & $1 \cdot 58$ & -.04 & .747 & $9 \cdot 39$ & $5 \cdot 20$ & .00 & פ \\
\hline $2 T$ & 21.90 & .548 & 19.98 & $-7 \cdot 101$ & $1.0 B$ & -.32 & .745 & 9.116 & $4 \cdot 5.5$ & .00 & .00 \\
\hline $37^{\prime}$ & 21.90 & .546 & $19.9 ?$ & -5.8392 & 1.74 & -.44 & - $7: 13$ & 9.39 & 4.31 & - $0 n$ & חח \\
\hline $4 \mathrm{~T}$ & $2 ? \cdot 30$ & .546 & $20 \cdot 04$ & -6.250 & 1.99 & -.71 & .728 & 9.37 & 4.55 & ח & .00 \\
\hline $5 T$ & 22.30 & .547 & $20 \cdot 311$ & $-7 \cdot 16 ?$ & 1.66 & - . - . - & .741 & 9.55 & 5.59 & . 00 & .00 \\
\hline
\end{tabular}

AUEKAGES: 707?1 PASELINE: W05400 000

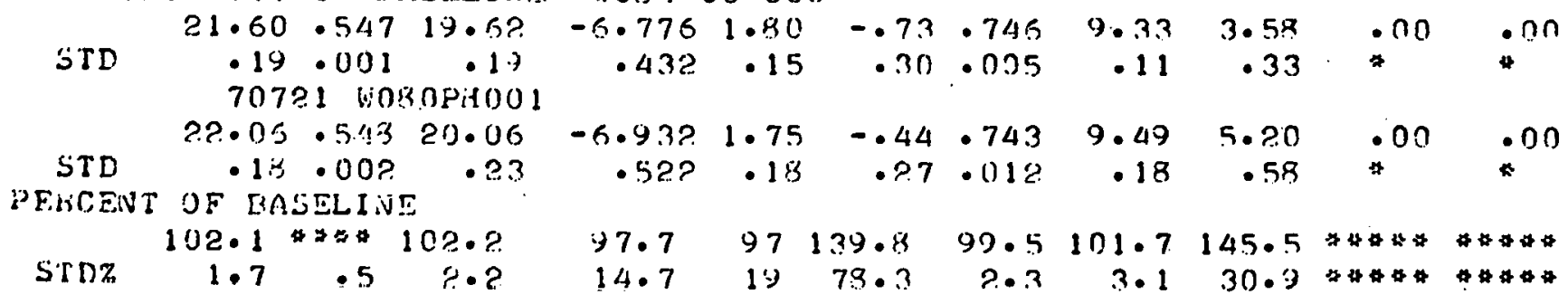




\subsection{Impurity/Grain Boundary Interactions}

Polycrystalline ingots or forms of silicon sheet containing. grain boundaries are potential low cost substrates for terrestrial solar cells. Thus a small part of this program is directed at elucidating the response of solar cells made on polycrystalline silicon to controlled additions of metal contaminants. As a first step in this study a baseline (no added metal impurity). $4 \Omega \mathrm{cm}$ p-type ingot was grown from a polycrystalline seed, wafered and then processed to solar cells using our standard sequence for $\mathrm{N}^{+} / \mathrm{P}$ devices.

The I-V lata fui Llie pulycrystalline ingor Wü7o, and concurrently processed single crystal baseline material appear in Table 16 . The designations $C, S$, and $T$ in the left hand column of the data printout refer to wafers from the center, seed and tang positions of the ingot. Overall the cells on the polycrystalline ingot are about $71 \%$ as efficient as the baseline material. However, material from the tang end of the ingot appears to give higher cell performance than that from the seed or center sections. This is perhaps consistent with the fact that material from the tang end contains some grains well over a $\mathrm{mm}$ in size, Fig 1 (a), while the first part of the ingot to freeze contained a much higher density of twins and associated dislocations, Fig. 1 (b). 
TABLE 16

Solar Cell I-V Data for Polycrystalline $4 \Omega \mathrm{cm}$ p-type Ingot.

70513 W076POLY00? $105400 \quad 000$

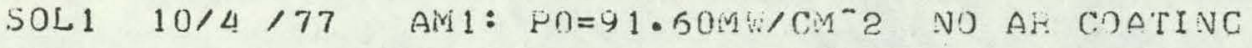

\begin{tabular}{|c|c|c|c|c|c|c|c|c|c|c|c|}
\hline D & I $\triangle C$ & voc & If & $L O C(10)$ & $\mathrm{v}$ & H & $F F$ & FFF & ori & PCnA & PCDI? \\
\hline $1 \mathrm{r} *$ & $2 ? \cdot 50$ & 553 & 20.18 & -6.315 & 1.98 & -.30 & 717 & 7.44 & .00 & . & .00 \\
\hline $1 \mathrm{~B}$ & $\because 1.70$ & 542 & 18.35 & $-4 \cdot 932$ & 2.77 & $-2 \cdot 11$ & . 698 & 8.58 & 0.99 & .00 & . nn \\
\hline$B$ & 22.30 & 549 & 20.39 & -7.257 & $1 \cdot 64$ & -.23 & .748 & 9.69 & $5 \cdot 46$ & . 00 & . $10 n$ \\
\hline $3 B$ & $22 \cdot 30$ & .550 & 20.28 & -6.777 & $1 \cdot 79$ & -.74 & .749 & $9 \cdot 72$ & $4 \cdot 55$ & .00 & .00 \\
\hline $4 \mathrm{E}^{*}$ & $21 \cdot 70$ & 543 & 19.03 & $-5 \cdot 208$ & 2.56 & -1.88 & .710 & 8.84 & $3 \cdot 51$ & .00 & - $n$ n \\
\hline$R$ & $22 \cdot 10$ & .548 & $20 \cdot 0.3$ & -5.535 & 1.84 & -.68 & .742 & 9.50 & $5 \cdot ? 0$ & .00 & . \\
\hline $\mathrm{C}$ & $15 \cdot 40$ & .508 & $16 \cdot 24$ & -6.051 & 1.97 & .43 & .580 & $6.7 ?$ & 1.04 & . 0 n & .00 \\
\hline C & 19.00 & .505 & $16 \cdot 18$ & -4. & $2.6 ?$ & -.50 & .646 & 6.55 & . 78 & . 00 & .00 \\
\hline 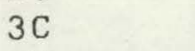 & 18.70 & $\cdot 509$ & 15.92 & $-5 \cdot 170$ & $2 \cdot 47$ & .59 & . 631 & .35 & .91 & .00 & - $\cap 0$ \\
\hline C & 8.50 & .510 & 16.34 & $-5.94 ?$ & 2.02 & -.07 & .690 & . 88 & $1 \cdot 04$ & • $0 n$ & .00 \\
\hline $5 \mathrm{C}$ & 18.80 & .506 & $16 \cdot 23$ & $-5 \cdot 452$ & 2.27 & .51 & .649 & 6.53 & - 78 & .00 & - 00 \\
\hline $6 \mathrm{C}$ & 19.40 & .507 & $16 \cdot 55$ & -5.092 & 2.51 & .11 & .640 & 5.66 & .91 & .00 & - ก \\
\hline $7 \mathrm{C}$ & $19 \cdot 30$ & .512 & 17.06 & -6.040 & 1.98 & .27 & . 685 & 7.16 & 1.04 & . 00 & .00 \\
\hline $3 \mathrm{C}$ & 19.80 & . $51 ?$ & $17 \cdot 17$ & $-5 \cdot ? .7 .2$ & 2.43 & -.80 & .674 & 7.23 & 1.04 & .00 & - on \\
\hline C & 19.00 & .504 & $16 \cdot 31$ & -11.927 & 2.63 & $-1 \cdot 33$ & . 668 & 6.77 & .91 & - & . $0 n$ \\
\hline $10 \mathrm{C}$ & .20 & .514 & 17.05 & -5.011 & 2.00 & -.39 & .703 & $7 \cdot 34$ & .91 & .00 & - \\
\hline $11 \mathrm{Co}$ & .00 & . 359 & $14 \cdot 12$ & $-4 \cdot 2.58$ & 2.36 & 3.45 & .469 & 3.39 & .39 & .00 & .00 \\
\hline $1 \mathrm{~S} \cdot *$ & 18.70 & .412 & $14 \cdot 40$ & $-4 \cdot 475$ & 2.50 & 3.40 & .500 & $4 \cdot 07$ & . 39 & .00 & .00 \\
\hline 25 & $19 \cdot 30$ & .514 & $17 \cdot 27$ & $-6 \cdot 241$ & 1.90 & -.47 & .715 & 7.51 & 1.17 & • 00 & .00 \\
\hline $3 s$ & $19 \cdot 40$ & .509 & $16 \cdot 15$ & $-4 \cdot 220$ & $3 \cdot 39$ & -2.65 & . $6 \Delta 5$ & $6 \cdot 74$ & . 78 & .00 & - \\
\hline $4 S$ & $18 \cdot 50$ & .492 & $15 \cdot 52$ & $-4 \cdot 592$ & 2.87 & $-1 \cdot 10$ & .635 & 6.12 & - 52 & $\cdot 00$ & - nn \\
\hline $5 S \cdot *$ & 17.90 & .427 & 13.47 & $-4 \cdot 310$ & 2.78 & $3.9 ?$ & .481 & 3.89 & - 26 & .00 & .00 \\
\hline 65 & $18 \cdot 60$ & .416 & $14 \cdot 15$ & $-4 \cdot 211$ & 2.80 & 2.85 & .497 & 4.06 & .26 & .00 & $.0 n$ \\
\hline $7 S$ & $18 \cdot 10$ & .481 & $14 \cdot 58$ & -3.997 & 3.56 & -1.83 & .597 & 5.49 & 3.90 & .00 & - \\
\hline $1 \mathrm{r}$ & $20 \cdot 70$ & .528 & 18.61 & $-6 \cdot 421$ & 1.86 & -.37 & . 722 & $8 \cdot 34$ & 1.30 & .00 & .00 \\
\hline $2 \mathrm{~T}$ & $19 \cdot 70$ & .519 & 57 & $-6 \cdot 170$ & 1.94 & -.26 & .707 & 7.64 & 1.04 & .00 & . nn \\
\hline $3 T$ & $20 \cdot 20$ & .515 & $17 \cdot 50$ & $-5 \cdot 130$ & 2.50 & $\cdot 1 \cdot 12$ & .679 & $7 \cdot 47$ & . 78 & .00 & - \\
\hline $4 \mathrm{~T}$ & 20.00 & .523 & 17.88 & $-6 \cdot 2.44$ & 1.92 & -.28 & .711 & 7.86 & $1 \cdot 17$ & • & - ח \\
\hline $5 \mathrm{~T}$ & 19.50 & .514 & $17 \cdot 26$ & -5.894 & 2.05 & -.39 & .698 & 7.40 & 1.04 & .00 & .00 \\
\hline $6 \mathrm{~T}$ & $19 \cdot 70$ & .517 & $17 \cdot 50$ & $-5 \cdot 970$ & $2 \cdot 03$ & -.51 & .705 & $7 \cdot 60$ & 1.04 & .00 & - 00 \\
\hline & 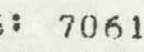 & & 2 & & 10000 & & & & & & \\
\hline \multirow{3}{*}{$\operatorname{stn}$} & 2P. 23 & .549 & $20 \cdot 2.3$ & -6.890 & 1.76 & -.55 & .746 & $9 \cdot 63$ & 5.07 & .00 & .00 \\
\hline & $\begin{array}{l}.10 \\
7061\end{array}$ & $\begin{array}{l}.001 \\
3 \quad 107\end{array}$ & $\begin{array}{c}15 \\
6 P O L Y 00\end{array}$ &.$? 65$ & .09 & .23 & .003 & .09 & - 38 & 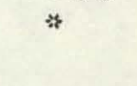 & 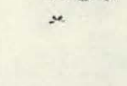 \\
\hline & $19 . ? 3$ & .505 & $16 \cdot 6 ?$ & -5.425 & 2.37 & -.35 & .666 & 6.88 & 1.06 & . 00 & . 00 \\
\hline \multirow{2}{*}{ PEECENT } & - 64 & .022 & 1.03 & $\cdot 731$ & $\cdot 48$ & 1.05 & - 050 & .89 & .67 & & \\
\hline & 86.5 & $\begin{array}{l}\text { SEL I. } \\
? .1\end{array}$ & & & & & & & & & \\
\hline ST D\% & $3 \cdot 3$ & $4 \cdot 2$ & $5 \cdot 8$ & 14.1 & 35 & 93.7 & $7 \cdot 1$ & 10.0 & 15. ? & $\pi \ldots \neq * *$ & $* * * 4 *$ \\
\hline
\end{tabular}




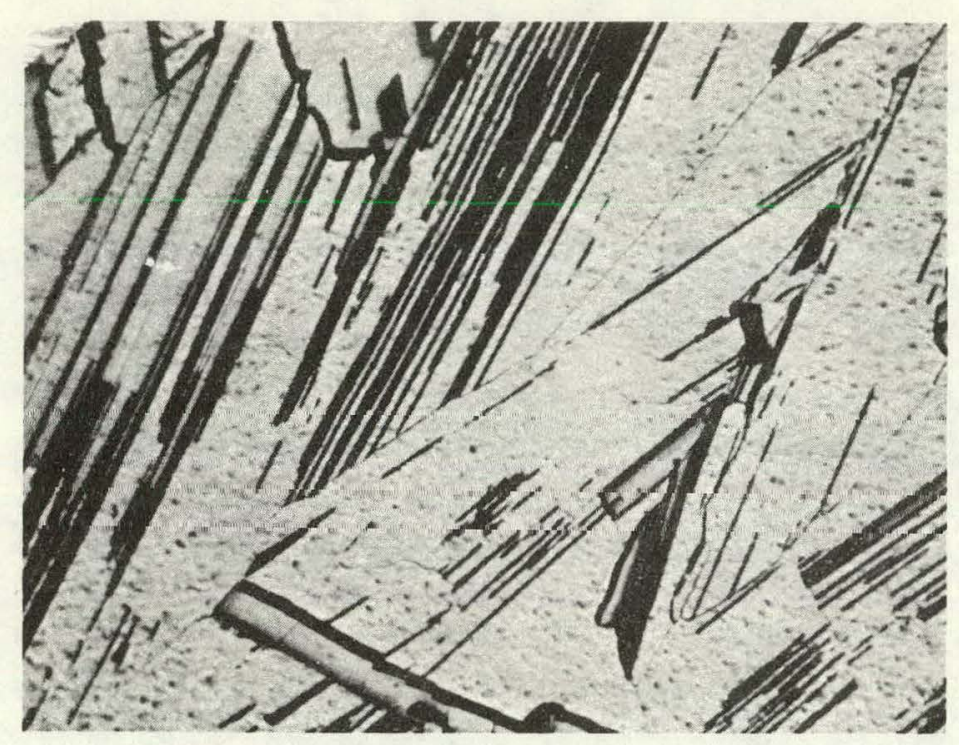

(a) Seed end

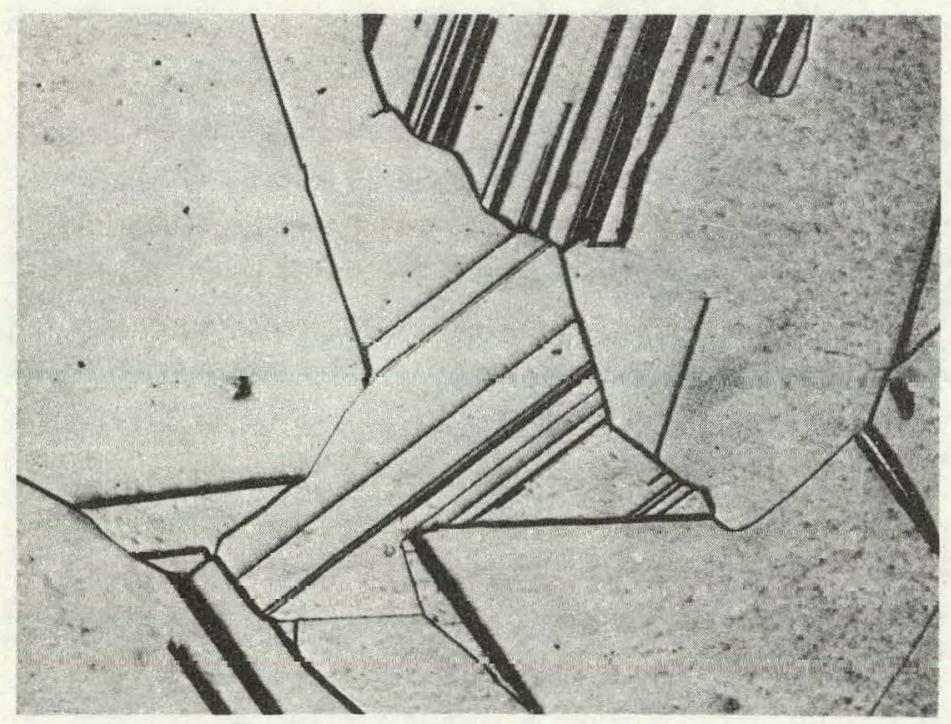

(b) Tang end

FIGURE 1 Sirt1 Etched Wafers from the Seed and Tang Ends of Polycrystalline Baseline Ingot w076. Reflected Light 32X 


\subsection{Refinement of Analytical Technqiues for Silicon Material and Solar Cells}

A major endeavor of tinis program is to quantify by the use of improved analytical techniques the relations between impurity-process interactions and solar cell performance. The mathematical model used to predict the behavior of solar cells containing single and multiple contaminants is one aspect of this effort. ${ }^{1,3}$ The correliation of data from thermally-stimulated measurements, refined PCD lifetime techniques and quantitative I-V measurement forms a second part of the approach. The following sections describe recent progress in the latter three areas.

\subsubsection{Thermally Stimulated Measurements}

During this reporting period, apparatus was modified to measure trap density and trapping parameters by both thermally stimulated current and capacitance measurements. Our initial attempts to desect deep level traps in solar cell material have been frustrated for reasons which are attributed to thermal deficiencies in the sample holder. These deficieicies are being corrected in a new sample holder which is now being built. The electronic components of the system have performed satisfactorily and have exhibited sufficient sensitivity to make the anticipated measurements.

\subsubsection{Recombination Lifetime Measurements (PCD)}

PCD lifetime measurements, as noted in Section 3.2, are being used to track the response of impurity-doped silicon to various thermal and gettering treatments which are employed during cell processing. To be meaningful the measurements must be carried out in a precise and reproduceable fashion that provide data which characterize the state of the material before and after processing. Thus we have carried out a sequence of tests to calibrate the laser-excited PCD apparatus and to assess possible error ranges in the measurement technique. 
Accurate PCD measurements requires that the surface recombination velocity, $s$, be known so that proper correction factors can be applied to the measured time constant, $\tau^{\prime}$, in order to obtain the bulk lifetime. In several previous attempts to determine.s,scatter in the data limited the confidence level that could be placed on the value of the surface recombination that was deduced.

The technique used to determine $s$ was to measure $\tau_{r}^{\prime}$ in a single piece of silicon as it was thinned by erching and Lu mathemar1cally find a value of $s$ which produced the best curve fit to the data. sillcon wafers with an initial thickness of $0.254 \mathrm{~mm}$ were used in these experiments since they were readily available. An insufficient number of data points were produced using these thin wafers to achieve good statistical analysis. Recently, silicon wafers with greater initial thickness, $2.01 \mathrm{~mm}$, have been used to refine this technique. The samples were thinned by a combination of lapping and etching. The uncorrected or effective lifetime for this series of measurements is shown in Fig. 2. The effect of higher modes was taken into consideration primarily by obtaining the average time constant between the $1 / 2$ and $1 / 4$ points of the maximum oscilloscope amplitude. As we will show later, this allowance for higher modes is insufficient for wafers with a bulk lifetime of $18 . y$ usec and a chlckness less than $0 . \dot{b i n}$. The curve in Fig. 2 can also be interpreted as the calculated bulk lifetime where $s$ is equal to zero.

Clearly, $s$ is grcazer than zeru since the curve benda downard for thinner silicon. The flattening of the curve for large values of $d$ is a consequence of the fact that $D_{n}\left(\zeta^{2} / d^{2}\right)$ becomes small compared to $1 / \tau_{r}$. The asymptotic value of the curve is equal to $\tau_{r}$. The value of $\tau_{r}$ can be bracketed by noting that the calculated value of $\tau_{r}$ $\left(2 \mathrm{~d}=2 \mathrm{~mm}, T_{r}=16\right.$ usec $)$ varies between the narrow range of 17.4 to 18.5 usec as $s$ is mathematically varied over the extreme range of $1 \times 10^{3}$ to. $2 \times 10^{4} \mathrm{~cm} / \mathrm{sec}$. 


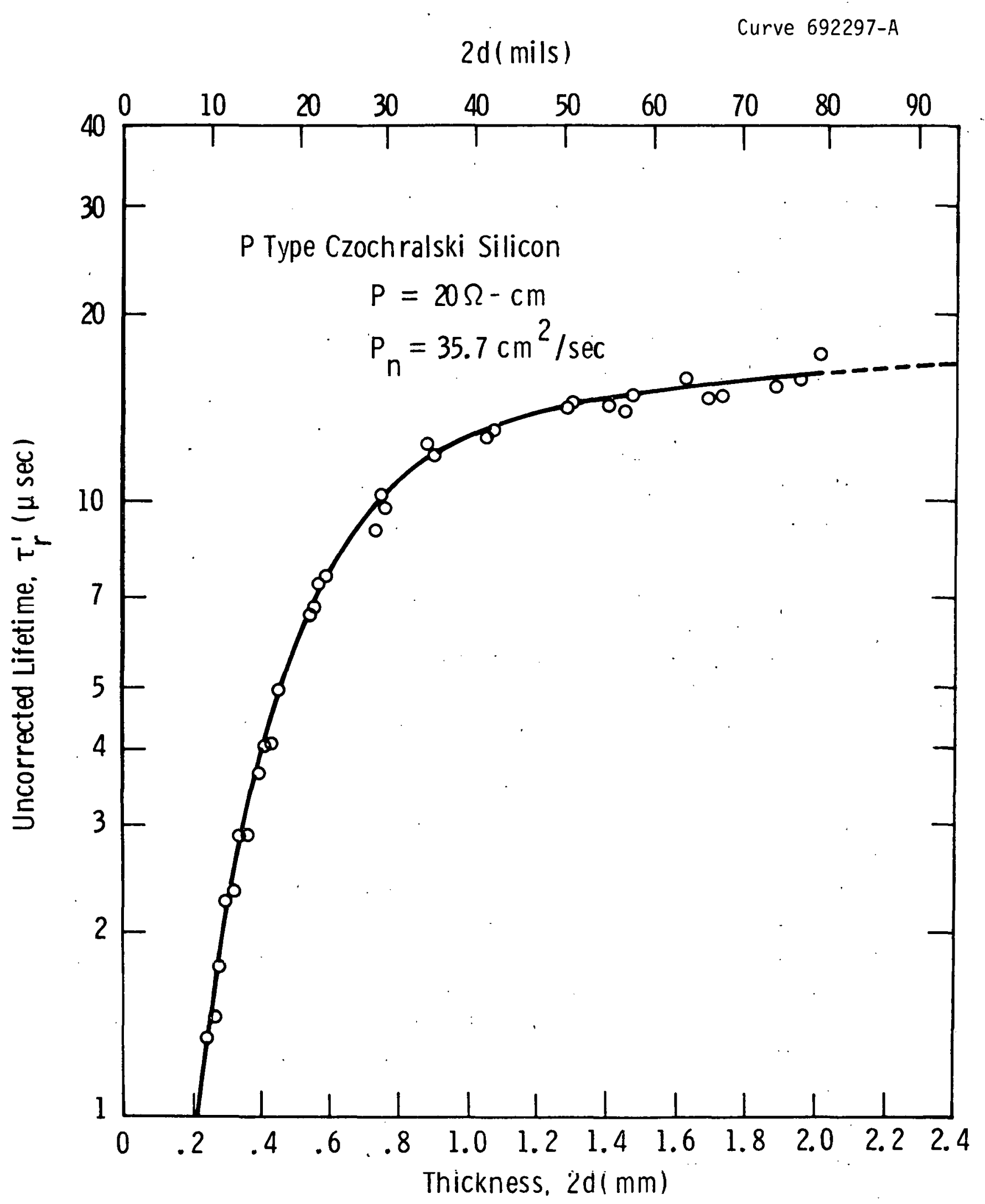

Fig. 2 Experimental data to be used in mathematically . determining the surface recombination velocity, $s$, on $p$ type silicon 
The curve shown in Fig. 3 represents the calculated value of $\tau_{r}$ when $s$ is assumed to be equal to infinity. Theoretically, this value of $s$ should produce a curve which increases monotonically as the thickness decreases. The curve can be analyzed by dividing it into two regions, (1) where surface recombination dominates (thickness greater than $0.5 \mathrm{~mm}$ ) and (2) where high order modes dominate (thickness less than $0.5 \mathrm{~mm}$ ). The shape of the curve beyond $0.5 \mathrm{~mm}$ indicates that $s$ is much less than infinity.

Fig. 4 was calculated with a șurface recombination veloc1ty aooumicd to bc $1.3 \times 10^{4} \mathrm{em} / 3 \mathrm{ec}$. Again, the curve leyund $2 \mathrm{~d}-0.8$ utu indicates that $s$ is less than $1.3 \times 10^{4} \mathrm{~cm} / \mathrm{sec}$. Through iteration of the data it was determined that the calculated lifetime became constant with thickness when $s$ was made equal to $5 \times 10^{3} \mathrm{~cm} / \mathrm{sec}$. Fig. 5 shows the best fit curve obtained for $s=5 \times 10^{3} \mathrm{~cm} / \mathrm{sec}$, considered accurate to $+0-30 \%$ based on the perception of slope change. The average value $(n=15)$ of $\tau_{r}$ obtained for $2 d$ greater than $0.8 \mathrm{~mm}$ is $18.6 \mu \mathrm{sec}$, and the standard deviation is 1.0 or a probable error of $0.68 \mathrm{usec}$.

The poorer accuracy in lifetime determination for $2 \mathrm{~d}$ values less than $0.7 \mathrm{~mm}(28 \mathrm{mils})$ is attributed mainly to the effects of higher modes in the spatial distribution of minority carriers. The effect of higher modes was discussed at length previously? In theory the effect can be reduced to insignificance (less than $5 \%$ ) by measuring the slope of the decay curve on the oscilloscope after the amplitude has decayed to less than $10 \%$ of the initial value. As a practical matter this is most difficult to do because of noise in the weak electrical signals, $\tau_{r}^{\prime}$ is more readily determined when the amplitude has decayed to $1 / 2$ or $1 / 4$ of its initial value. This compromise yields values for $\tau_{r}^{\prime}$ which are less than the true value for the desired zero order mode. The measurement error may be quite small but because of differential nature of Equation $3.6-1$, it may be magnified manyfold

$$
\frac{1}{\tau_{r}}=\left[\frac{1}{\tau_{r}}-D_{n}\left(\frac{\zeta}{d^{2}}\right)\right]
$$


Curve 692295-A

$2 d$ (mils)

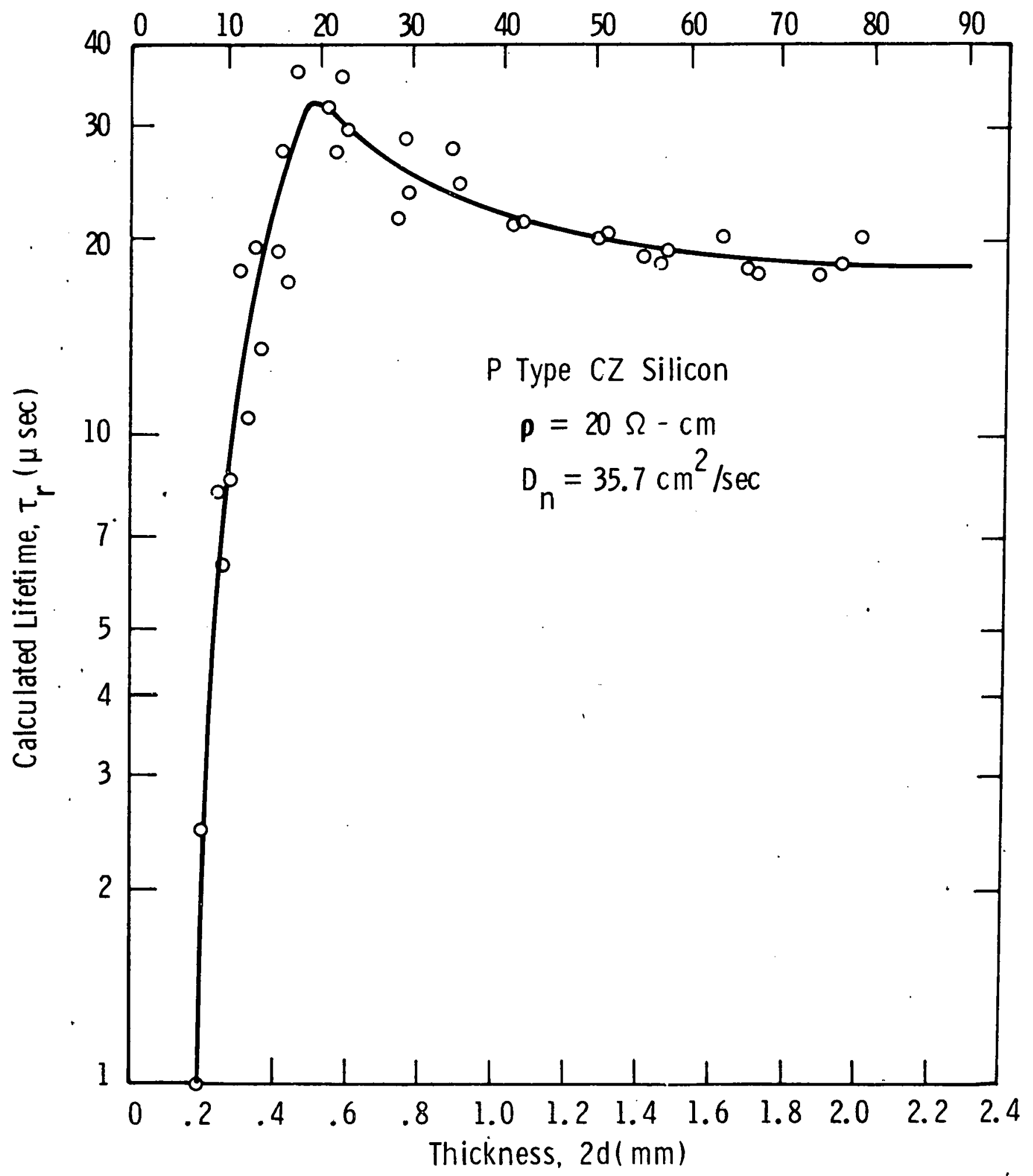

Fig. 3 Calculated bulk'lifetime as a function of thickness for the case when $s$ is assumed to be equal to infinity 
Curve 692298-A

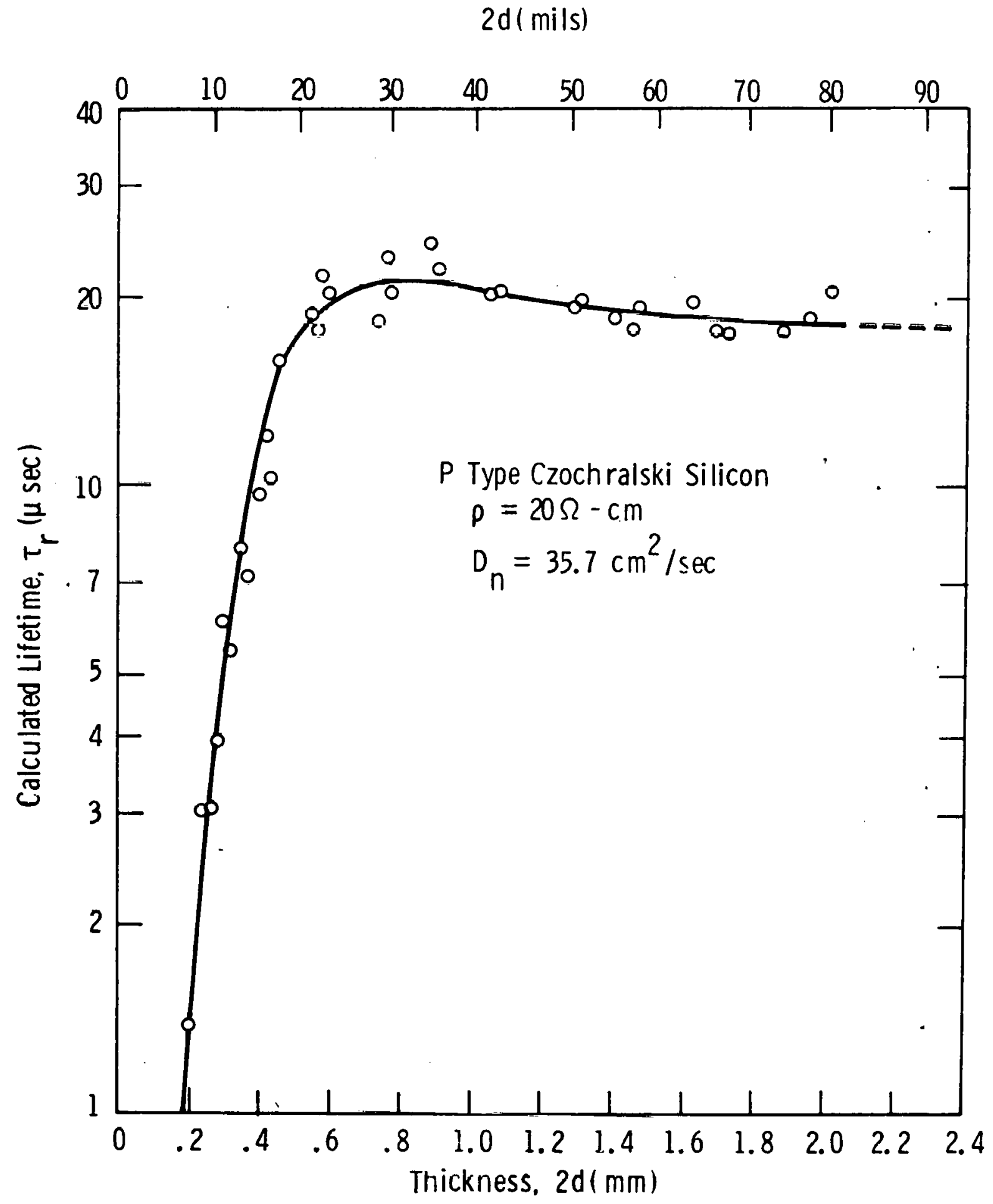

Fig. 4 Calculated bulk lifetime as a function of thickness

for the case when $s$ is assumed to be equal to

$1.3 \times 10^{4} \mathrm{~cm} / \mathrm{sec}$ 
Curve 692296-A

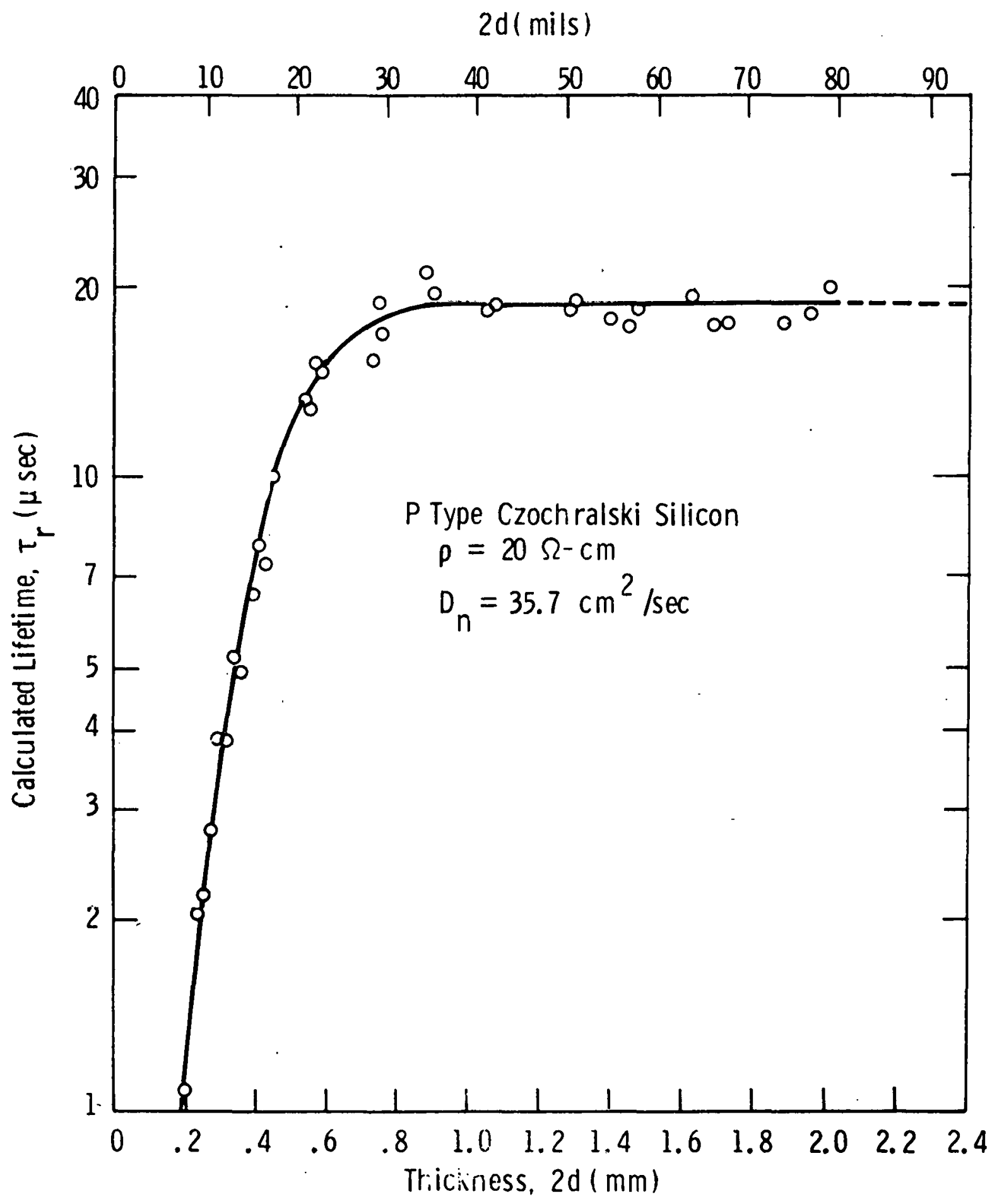

Fig. 5 Correctly chosen value of $s=5 \times 10^{3} \mathrm{~cm} / \mathrm{sec}$ yields a constant value for $\tau_{r}=18.6 \mu \mathrm{sec}$ as thickness is varied above $0.8 \mathrm{~mm}$ 
and yield large errors in the calculated value of $\tau_{r}$. This type of error always produces a calculated value of ${ }^{\tau}{ }_{r}$ which is less than the true value as indicated by the curves in Figures 2, 3, and 4. The error increases rapidly for thin wafers with high lifetime, since $D_{n}\left(\xi_{0}^{2} / d^{2}\right)$ may be nearly equal to the value of $1 / \tau_{r}^{\prime}$. For convenience, the factor $D_{n}\left(\xi_{0}^{2} / d^{2}\right)$ is often referred to as the surface rate of the fundamental. mode, $1 / r_{000^{\circ}}$ Likewise, the factor $1 / \tau_{\mathrm{r}}$ is referred to as the bulk rate. The ratio of the bulk rate to the surface rate, $\left(1 / \tau_{\mathbf{r}}\right) /\left(1 / \mathbf{r}_{\text {ooo }}\right)$, as described in the last quarterly report, was shown by Blakemore and Normura ${ }^{4}$ to be a principal factor in determining the accuracy of PCD measurements. A ratio of 1.0 or greater was recommended by them to achieve accuracies of about $+0-50 \%[\mathrm{~N}(\tau) / \mathrm{N}(0)=0.50]$; ratios less than 0.1 are considered to produce unreliable data. Fig. 6 shows a plot of the bulk lifetime, ' $r$, necessary to achieve a ratio of 1.0 , as a function of wafer thickness for p-type silicon. The curve indicates that a silicon specimen with a thickness of $0.65 \mathrm{~mm}$ is necessary for accurate PCD measurements when the bulk lifetime is $18.6 \mathrm{usec}$. This corresponds very closely with the point where large deviations begin to appear in Fig. 5. A superposition of the curve in Fig. 6 over that shown in Fig. 5 shows that the error for thin wafers is almost entirely due to the effects of higher modes. The balance of the errur results from the fact that the specimen becomes "pillow" shaped in cross-sectiun after repeated etching. The primary effect of the pillow shape is to enhance the effect of the higher modes since the wafer is thinner than the measured value $2 \mathrm{~d}$ near the edges.

'l'he tentative requirement chat $\left(1 / \mathrm{T}_{\mathrm{r}}\right) /\left(1 / \mathrm{s}_{\text {ooo }}\right)$ be equal to or greater than 1.0 resulted from the fact that $\tau_{r}^{\prime}$ was determined by measuring the time required for the signal to decay from $1 / 2$ to $1 / 4$ of its initial amplitude and then dividing by $1 n 2$. A minimum ratio of 0.1 would be more desireable in order to allow measurements of high lifetime on thin specimens. Accurate measurements, with a ratio of 0.1 , are possible if the value of $\tau_{r}^{\prime}$ is obtained after the signal has decayed to less than $10 \%$ of the initial value. The ability to make measurements in 
Curve 692300-A

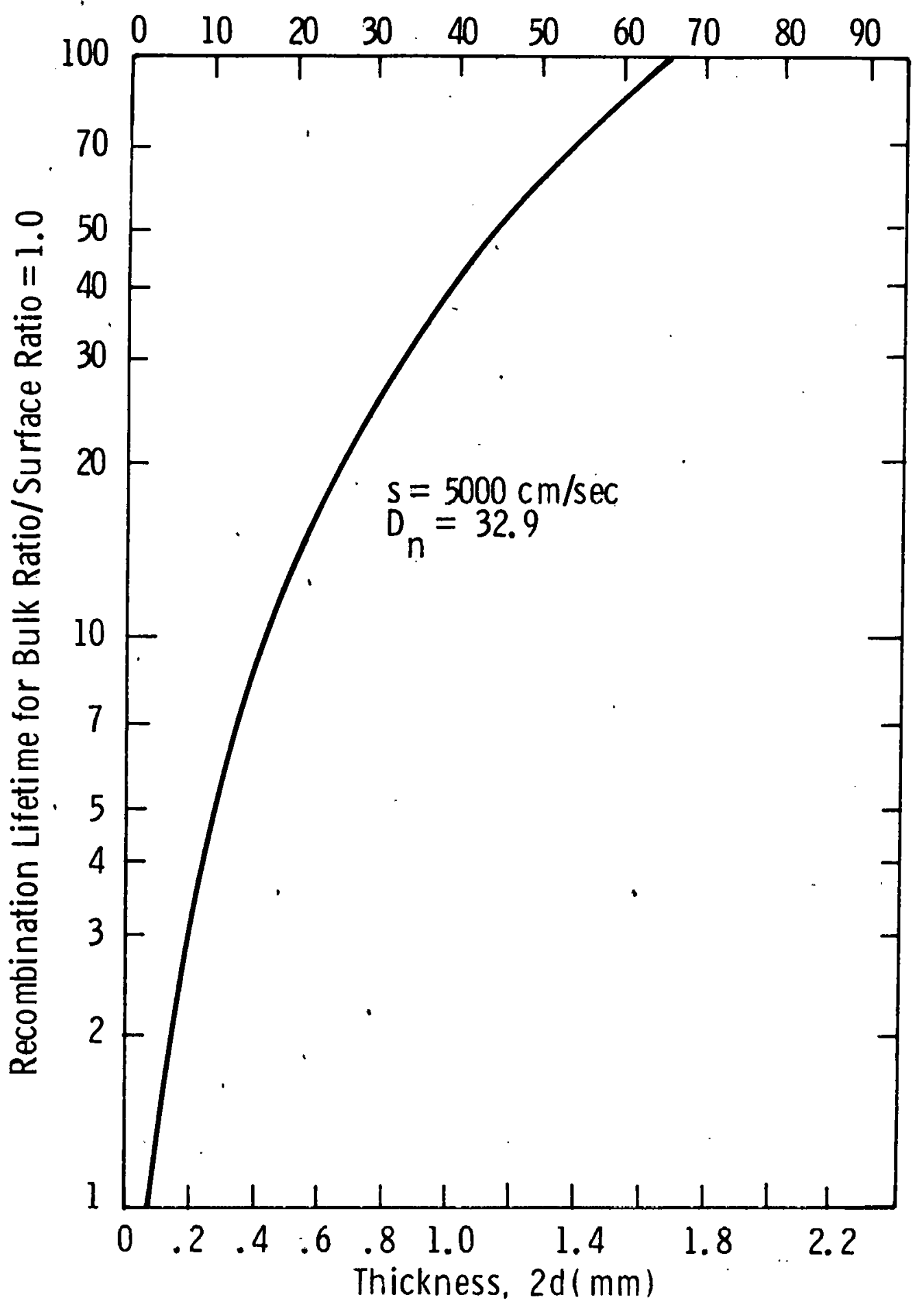

Fig. 6 Maximum recombination lifetime' which can be accurately measured (ratio $=1.0$ ) on p-type silicon by PCD measurements as a function of sample thickness. 
this region of the decay curve is determined by the signal to noise ratio. The signal to noise ratio can be improved by increasing the test current in the specimen, $I_{t}$ and/or increasing the number of minority carriers at $t=0$. The effect of increasing these two parameters was investigated experimentally to obtain practical limits for each.

The test current, $I_{t}$ produces an electric field within the silicon sample which "sweeps" the minority carriers toward the ohmic contacts where they will experience enhanced recombination. The effect of electric field on the corrected recombination liferime is shuwl in Fig. 7. The slight increase in the measured litetime is contrary co that expected by simple theory, which indicates that the measured lifetime should be reduced by the effects of electric field. The deviation from simple theory is due to some rise in the specimen temperature by $I^{2} R$ heating.

Simple theory predicts that the critical field may be calculated from the expression ${ }^{5}$

$$
\overrightarrow{\mathrm{E}}_{x}-1 . c x \ll \sqrt{\frac{4 D_{n}}{\mu_{n^{2} \tau_{r}}}}
$$

The critical field calculated for the measured sample is 1.98 volts/cm. No curvature is noted in Fig. 7 beyond this critical point. The reason for this behavior is that the ohmic contacts are spaced $1.0 \mathrm{~cm}$ apart whereas the laser pulsed light is applied to a length of only $0.5 \mathrm{~cm}$. Therefore, the carriers can experience considerable drift before reaching the ohmic contacts. The calculated lifetime in Fig. 7 for an electric field of zero is $20.3 \mathrm{\mu sec}$. This is higher than the value of $18.6 \mathrm{\mu sec}$ shown in Fig. 5. The reason as discussed below, is that the injection level was increased from $4 \times 10^{-3}$ to $1.1 \times 10^{-2} \mathrm{~cm}^{-3}$. 
- Curve 692294-A

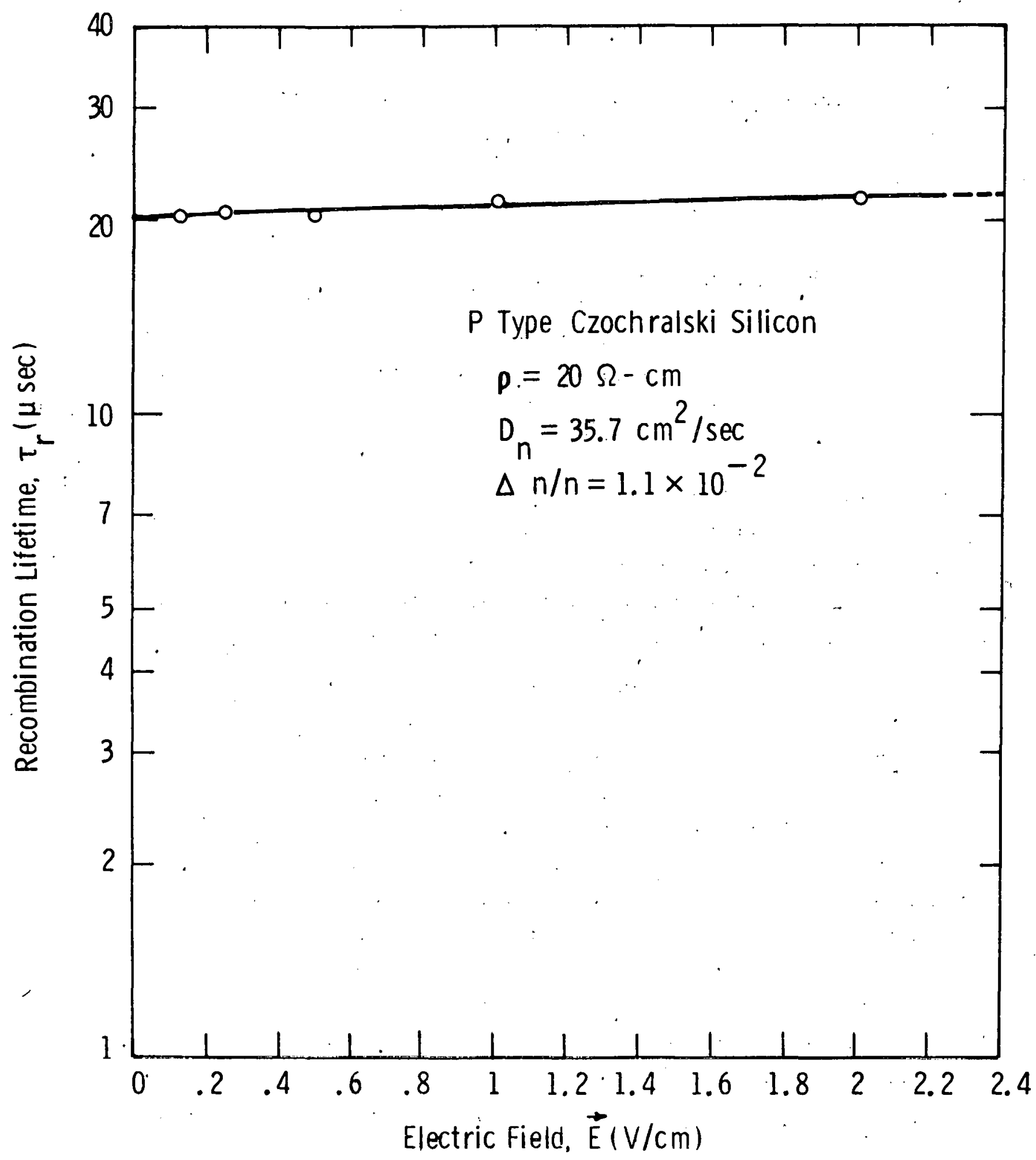

Fig. 7 Effect of electric field, $\vec{E}$, produced by the specimen test current, $I_{t}$, on photoconductivity decay measurements 
The effect of injection level on PCD measurements is shown in Fig. 8. The injection ratio was calculated by ${ }^{6}$

$$
\begin{aligned}
& \Delta \mathrm{n} / \mathrm{n}=\frac{\mathrm{b}}{\mathrm{b}+1} \frac{\Delta \mathrm{v} / \mathrm{v}}{2 / \ell_{0}-\Delta \mathrm{v} / \mathrm{v}} \\
& \text { Where: } \Delta n / n=\text { ratio of injected minority carriers to majority } \\
& \text { carriers } \\
& \dot{b}=\text { mobility ratio of majority to minority carriers } \\
& \Delta \mathrm{v} / \mathrm{v}=\text { relative change in photovoltage to dark voltage } \\
& y . / 40=\text { ratio ot iliuminated lengeh to the cocal eleclalial } \\
& \text { length. }
\end{aligned}
$$

A ratio of 1.0 for $\Delta \mathrm{n} / \mathrm{n}$ is defined as high level injection and ${ }^{\tau} \mathrm{r}$ asymptotically approaches $\tau_{\text {no }}+\tau_{\text {po }}$ as the injection level increases. The shape of the curve in Fig. 8 is typical of that obtained by other experimenters. This curve has considerable significance for the design of multi-sun solar cells as well as in miking accurate PCD measurements.

In summary, the laser-excited PCD equipment is now calibrated for measurements on p-type silicon. Errors due to electric field will be less than $5 \%$ when the field is limited to 1.0 volt $/ \mathrm{cm}$. Errors due to injection level will be less than $5 \%$ when tle injertion ratio is limited to $5 \times 10^{-3}$. Errors due to the effects of higher modes $\left[\tau_{r}^{\prime}=\left(\tau_{1 / 2}\right.\right.$ $\left.\left.\tau_{1 / 4}\right) / \ln 2\right]$ can be kept, in the range $+0-50 \%$ when the ratio $(1 / \tau) /\left(1 / \mathrm{r}_{000}\right)$ is greater than 1.0. The leeway allowed in the electric field and injection level over that used in obtaining Flgures 2-5 indicates that $\tau_{r}^{\prime}$ can be accurately determined after the higher modes have decayed or the signal has decayed to less than $10 \%$ of initial signal at $t=0$. This techrique, to be evaluated shortly, will increase the accuracy and/or allow lower ratios of bulk rate to surface rates. 'l'he equipment will soon be applied in the processing studies described in section 3.2 . 


\subsubsection{Evaluation of Impurity/Processing Effects on Solar Cell}

Performance by Means of Quantitative I-V Curve Analysis.

Although the mathematical model we recently developed 1,3 predicts quite well the effect of multiple contaminants on silicon solar cell efficiency, it cannot, because of its phenomenological nature, be used to analyze a number of process-induced effects which influence solar cell behavior. Nor can the nonlinear effects of impurities such as copper, nickel and iron be completely explained within the context of the model. For this reason we set out to develop a practical analytic and experimental technique which would allow separation of the junction and base region effects from each other and from effects due to contacting and resistance phenomena. The technique, which represents a synthesis of our ideas as well as some which have. appeared over the years in the literature, is described below. Because we believe the approach provides a powerful tool for assessing both impurity and process dependent effects on solar cells and because what has been reported in the literature appears in diverse sources, we have discussed the technique in a tutorial fashion, and with considerable detail below. The work was primarily conducted as part of this study but for completeness the description is supplemented by data developed on an internallyfunded Westinghouse program. 
Curve 692299-A

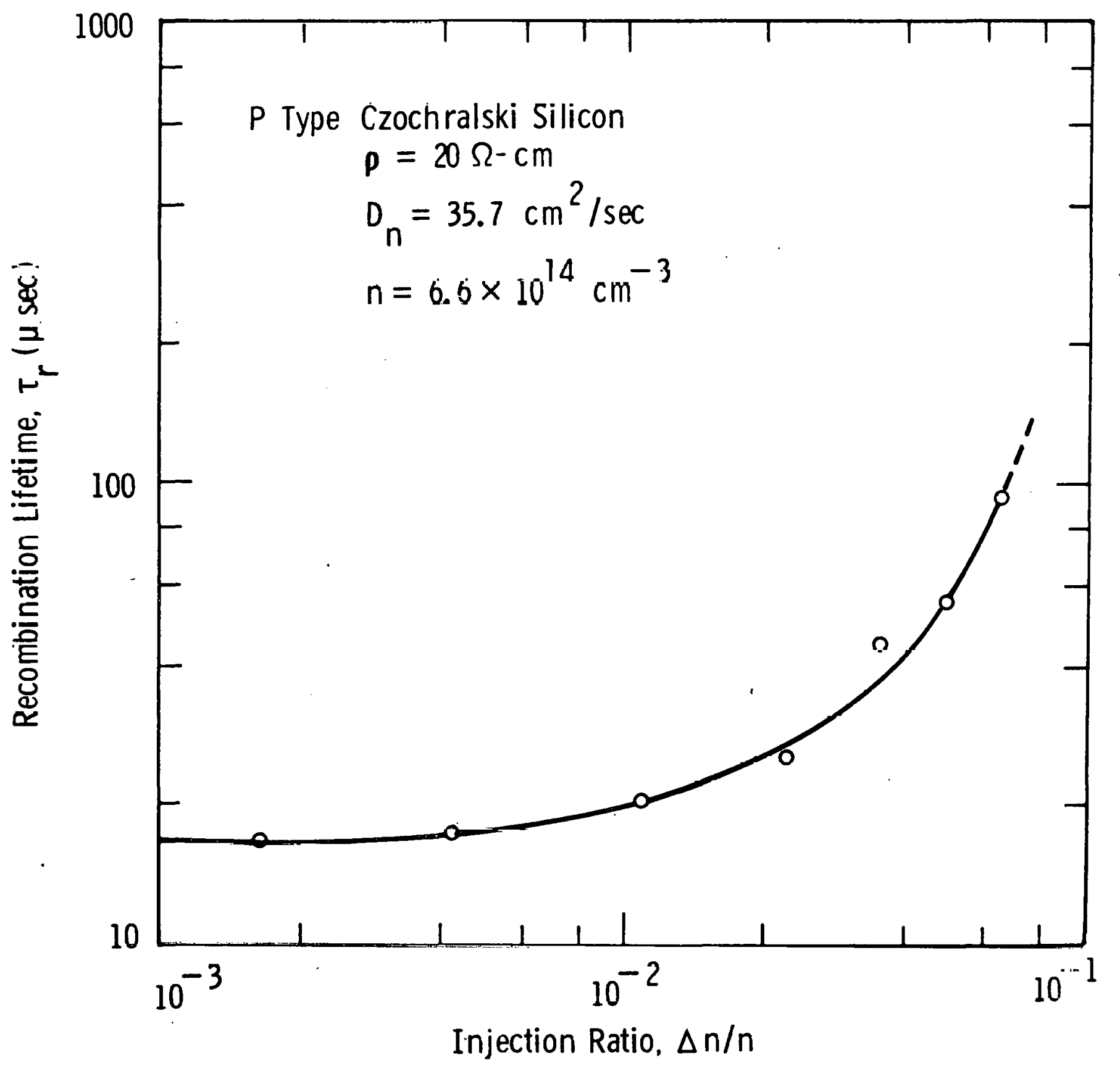

Fig. 8 Effect of pulsed light irradiance on photoconductivity decay measurements 


\subsubsection{1 .Review of Diode Current-Voltage Characteristics}

In a diode, forward bias reduces the barrier height across the junction allowing the majority carrier; to cross the junction and diffuse as minority carriers before recombining. This bulk diffusion current is expressed by the equation.

$$
I_{B}=I_{o_{1}}\left(e^{q v / k t}-1\right)
$$

where Io $_{1}$ is the reverse saturation current given by

$$
\mathrm{Io}_{1}=\mathrm{AqN}_{i}^{2}\left[\frac{1}{\mathrm{i} a} / \frac{\mathrm{D}_{n}}{\pi_{\mathrm{n}}}+\frac{1}{\mathrm{~N}_{\mathrm{D}}} / \frac{\mathrm{D}_{\bar{i}}}{\pi_{\mathrm{e}}}\right]
$$

In a real diode some carriers are lost in the depletion region as a result of recombination, trapping, and other conduction mechanisms such as, tunneling or field induced emission. current is expressed as $\stackrel{9}{,} 10$

$$
I_{1}=I_{2}\left(e^{c_{i} v / n k T}-1\right)
$$

where $n$ is: 2

There is always an intrinsic series resistance ${ }^{1}{ }^{1}$ present in a diode. In a solar cell the major source of this is the sheet resistance of the diffused region, while other less important sources are resistances of the base region and the contacts. Thus the terminal voltage differs from the junction voltage by $I_{s}$ drop.

Shunt or leakage current results from the imperfections which bridge the junction, especially at the edges where ohmic surface conduction paths are easily formed. This effect is represented by a physical shuntresistance across the junction and a current. 


$$
I s h=\frac{V_{d} 2}{R_{s h}}
$$

Figure 9 shows an equivalent circuit of a real diode which includes all the above effects which mathemat: $:$ ally are described by equation 3.6 .8

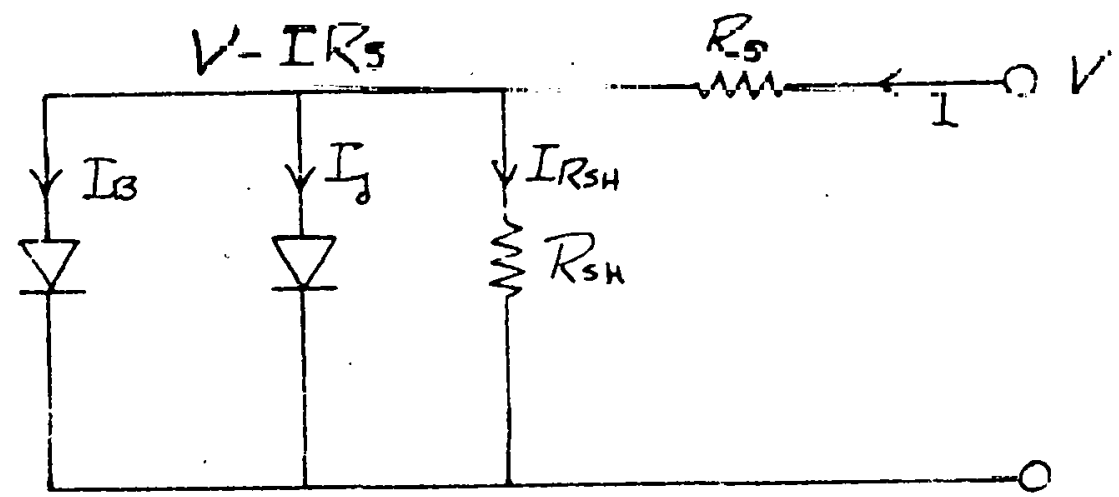

FT.G. 9 Equivalent sirçuit of a real diode.

$$
\begin{aligned}
& I_{d}=I_{B}+I j+I s h \\
& =I o_{1}\left(e^{a\left(V-I R_{s}\right) / k t}-1\right)+I_{2}\left(e^{q\left(V-I R_{s}\right) / n k t}-1\right) \\
& +\frac{V-I R s}{R s h}
\end{aligned}
$$

When a diode is used as a solar cell the terminal current (I) is given by

$$
I=I_{L}-I_{d}
$$


where $I_{L}$ is the current generated by the 1ight. Thus for a given 11jht-source, diode parameters $\mathrm{R}_{\mathrm{s}}, \mathrm{R}_{\mathrm{sh}}, \mathrm{Io} \mathrm{o}_{1} ; \mathrm{lo} \mathrm{o}_{2} \mathrm{n}$ and $\tau$ control the performance of the solar cell.

\subsubsection{Procedure for Separating Diode Parameters from the Experimenta1}

\section{Dark I-V Data}

It should be recognized that equation 3.6 .8 is a lumped approximation to the real device in which the distributed parameters may have appreciable anisotropy. Considerable experimental evidence has shown the approximation to be highly accurate except for cases of extreme anisotropy. It is therefore important for our purpose, to be able to dissect the measured current-voltage characteristic into its constituent functions as given in equation (3.6.8).

The first step in this process is to remove the $R_{s}$ and $R_{s h}$ terms. This is done by transforming the voltage and current variables:

$$
\begin{aligned}
& V^{\prime}=V-I R_{S} \\
& T^{\prime}=I-\frac{V-I R_{S}}{R_{S h}}
\end{aligned}
$$

The result of this process is illustrated in Fig. 10a for a device with large $R_{S}$ and $R_{S h}$ effects. Of course $R_{S}$ and $R_{s h}$ must be known and the methods of obtaining them are described in the following sections.

The second step ${ }^{13}$ involves separating the functions $I_{B}$ and $I_{j}$. This separation can be carried out exactly by computational methods, however, an acceptably accurate approximation can be obtained by the method illustrated in Fig. 10b. The current data points of $I_{B}(V)$ are obtained as follows: 


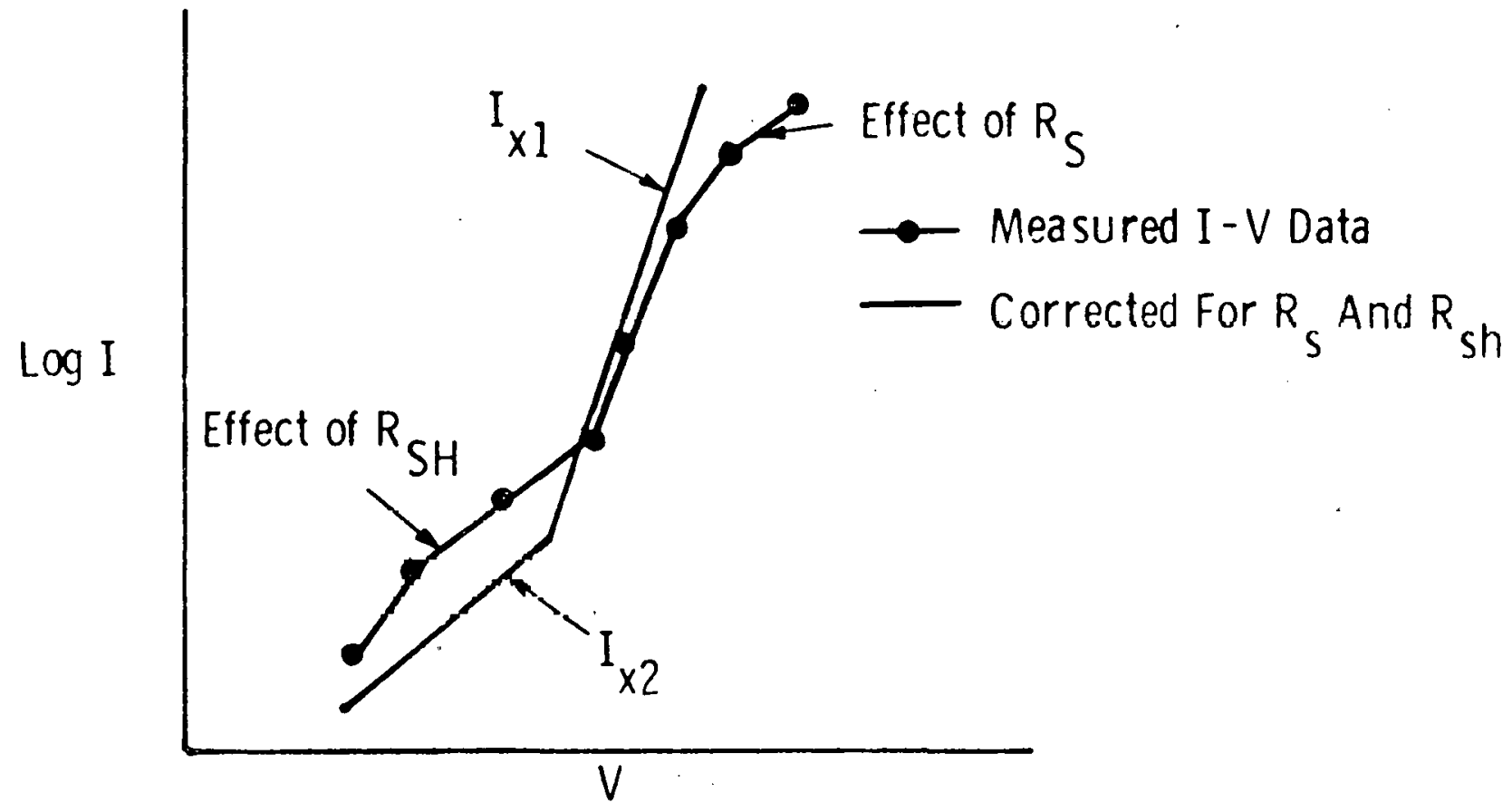

Fig. 10a

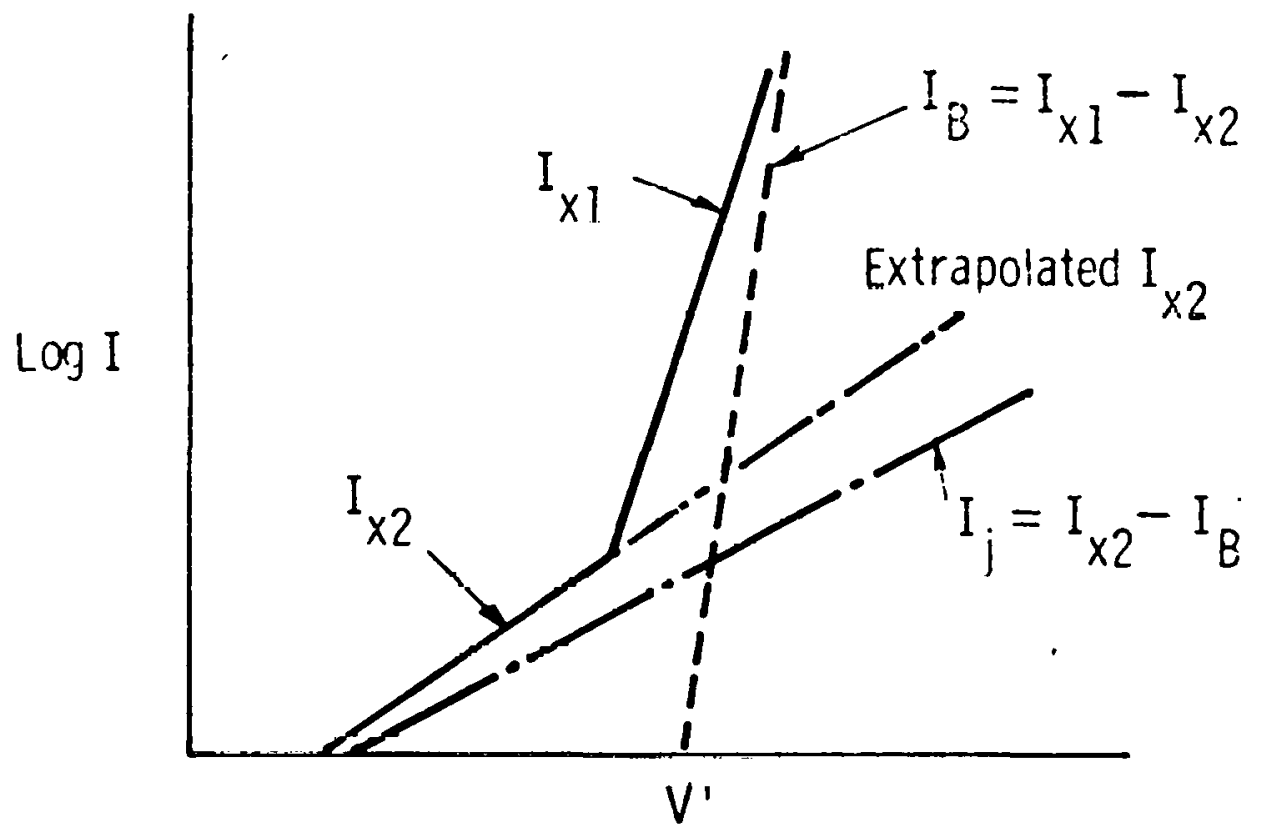

Fig. $10 \mathrm{~b}$

Fig. 10 Schematic Diagram of the Procedure for Transforming the Measured I-V Data to Separate the Bulk $\left(I_{B}\right)$ and Junction $\left(I_{f}\right)$ Components. 


$$
I_{B}(V)=I_{X 1}(V)-I_{X 2}(V)
$$

$$
\text { then } \quad I_{j}(V)=I_{X 2}(V)-I_{B}(V)
$$

As shown in the illustration $\log I_{B}$ plots as a straight line with slope of $q / k t$ and intercept Io ${ }_{1}$ while $I_{j}$ has a slope $q / n k t$ with $n \geqslant 2$ and intercept $\mathrm{Io}_{2}$. Thus we have now detcrmined all the parameters of equation 3.6 .8 except $R_{s h}$ and $R_{S}$ which are dealt with next. 


\subsubsection{Parametric Effects on Cell Performance}

The sources of the parameters which influence solar cell performance have been discussed. In this section we consider:

a) How the parameters influences the solar cell performance.

b) Quantitative determination of the parameters by $I-V$ measurements.

c) Discussion of relcvant experimental data showing the role. . of the parameler's In "guud" ard "uad" devices.

\section{A. SHUNT RESISTANCE}

Effect of Shunt Resistance on Solar Cell Efficiency

For the purposes of this discussion it is sufficient to describe the solar cell as a single diode, and neglect the effects of $I_{f}$ and $R_{s}$. Figure 11 shows an equivalent circuit of a solar cell with shunt resistance. In the figure $I_{d}=I_{B}+I_{\text {sh }}$ and $V=V_{d}$

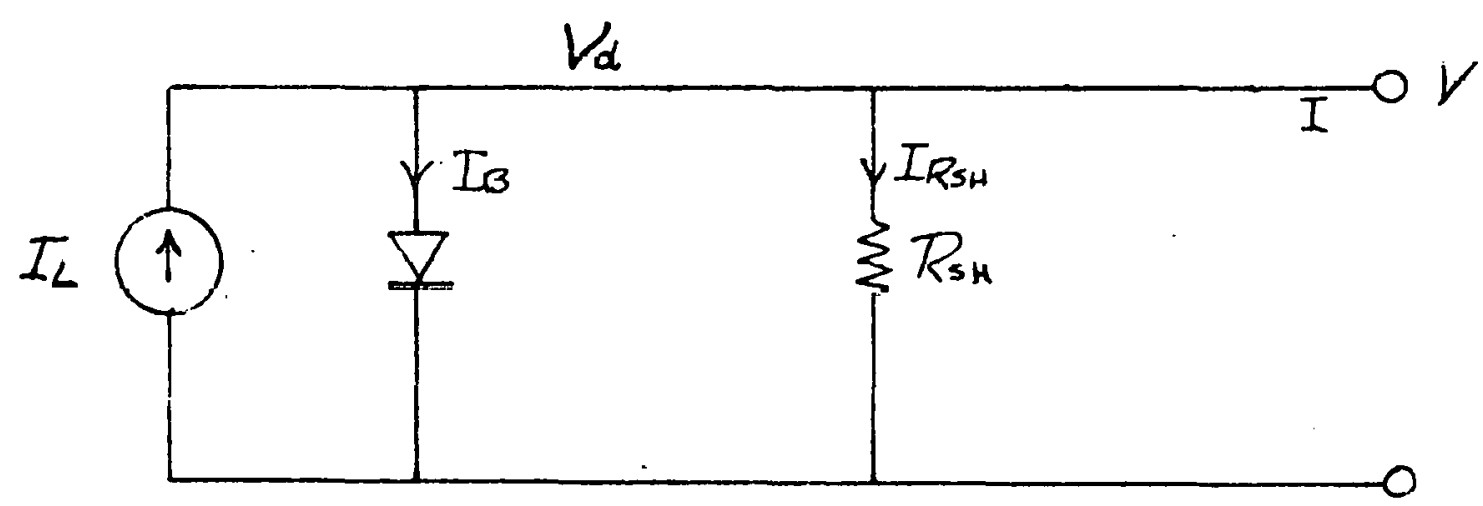

Fig. 11 Equivalent Circuit of a Diode with Short Resistance Only 


$$
I_{B 1}=I_{L}-I \text { with no shunt resistance }
$$

while $I_{B 2}=I_{L}-I-I_{s h}$ with the shunt resistance

For a given terminal current $I, I_{B 2}<I_{B 1}$,

$$
\begin{aligned}
& \text { since } \quad I_{B}=\text { Io }\left(e^{q^{V} d / n k t}-1\right) \\
& V_{d 2}<v_{d 1} \text { or } v_{2}<v_{1}
\end{aligned}
$$

This will move the peak power point inward and reduce the fill factor, open circuit voltage ( $\left.V_{o c}\right)$ and the cell effictency. Figure 12 depicts solar cell I-V carries with various shunt resistances. These data were obtained by putting various shunt resistance across the device. Figure 12 dictates that shunt resistnace will significantly affect the solar cell efficiency only when $R_{s h} \leq 100 \Omega$. For $R_{s h} \geq 300 \Omega$ the effect is negligible. Short circuit current ( $\mathrm{sc}_{\mathrm{sc}}$ ) is not affected appreciably until $\mathrm{R}_{\mathrm{sh}}<1 \Omega$.

\section{Determination of Shunt Resistance}

The effect of $R_{\text {sh }}$ is more apparent in the low current domain of I-V data. It is quite clear from equation 3.6 .8 and Figure 10 a that the shunt resistance can be identified as a curvature in the low current segments of the $\log I$.vs. V plot (if the effect of $R_{s h}$ is appreciable). Shunt resistance can be experimentally determined from the reverse dark I-V measurement. In the reverse bias

$$
\text { if } \begin{gathered}
R_{s h}=x, I=-I_{0} ; \text { however if } \frac{V}{R_{s h}} \gg I_{0} \\
\text { then } R_{s h}=\frac{\Delta V}{\Delta I}
\end{gathered}
$$


For this case

$$
I_{d}=I_{B}=I_{0}\left(e^{q\left(V+I R_{s}\right) / k T}-I\right)=I_{0}\left(e^{q V d / k T}-1\right)
$$

$$
\begin{aligned}
& \text { while } \quad \mathrm{V}=\mathrm{V}_{\mathrm{d}}-\mathrm{IR}_{\mathrm{S}} \quad \text { with series resistance, } \\
& \text { and } \quad \mathrm{V}=\mathrm{V}_{\mathrm{d}} \quad \text { without series resistance. } \\
& \text { since } L=L_{L}-I_{d}
\end{aligned}
$$

Therefore for a fixed $I, V_{d}$ will be the same and the terminal voltage (V) will be smaller by the amount $I_{S}$ when the series resistance is present. This will move the peak power point inward and reduce the fill factor and the efficiency of the cell. Figure 14 shows the I-V characteristics of the solar cell with various series resistances. $R_{S}$ as low as $I \Omega$ tends to affect the cell efficiency. $R_{s}>3 \Omega \mathrm{cm}$ significantly deteriorates the performance of solar cell.

Series resistance does not affect the open circuit voltage . ( $V_{o c}$ ) because there is no voltage drop across $R_{s}(I=0)$. Serfes resistance affects $I s c$ because in the short circuit case $I_{L}$ is divided between $R_{S}$ and diode impedance.

\section{Identification and Determination of Series Resistance}

The series resistance effect is more pronounced in the high current domain. Neglecting (or subtracting) the contribution from $I_{f}$ and $I_{s h}$, the diode current is written as

$$
\begin{array}{r}
I_{d}=I_{B}=I_{0}\left(e^{\eta\left(V-I R_{s}\right) / k T}-I\right) \\
\log I=\log I_{0}+\frac{a}{2.3 k T}\left(V-I R_{s}\right)
\end{array}
$$




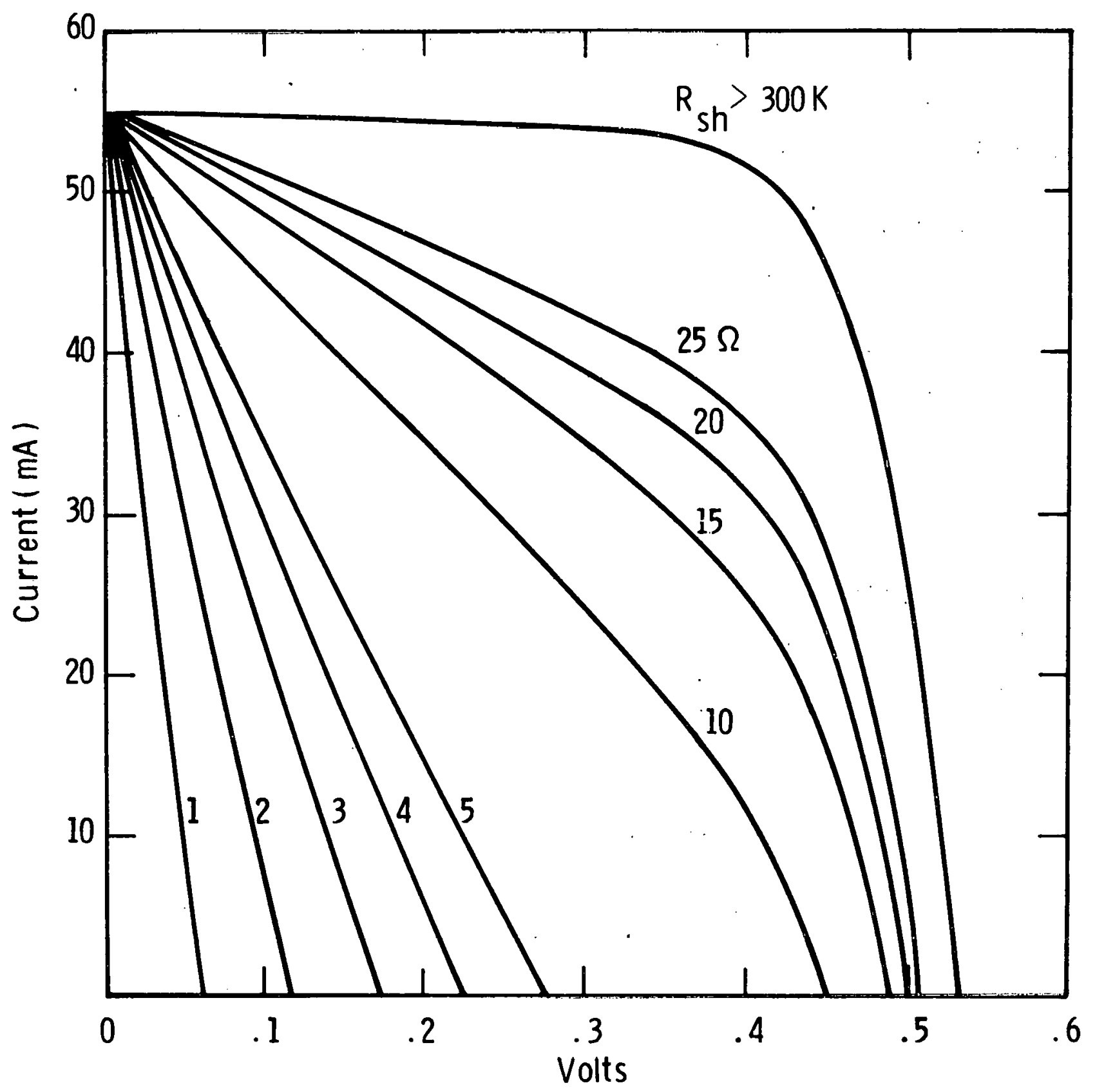

Fig. 12 The Effect of Shunt Resistance on Solar Cel1 I-V Characteristics. 
We found that in good devices ( $\mathrm{n}>9 \%$ without AR coating) $\mathrm{R}_{\text {sh }}$ is usually $>300 \mathrm{k} \Omega$ and therefore has insignificant influence on the cell performance (section 3.5.3.a). Even in very bad devices shunt resistance was $>20 \mathrm{k} \Omega$ and can therefore, not account for the poor efficiency observed. Rsh was so high in all the devices and $I_{j} / I_{s h} / v>$ 10 that no curvature in the low current segment was ever observed. Thus our data indicate we can neglect the affect of shunt resistance for all practical purposes.

\section{B. SERIES RESISTANCE}

Effect of Series Resistance on Solar Cell Efficiency

Figure 13 shows the equivalent circuit of the solar cell with series resistance. The effect of $I_{j}$ and $I_{s h}$ are again neglected.

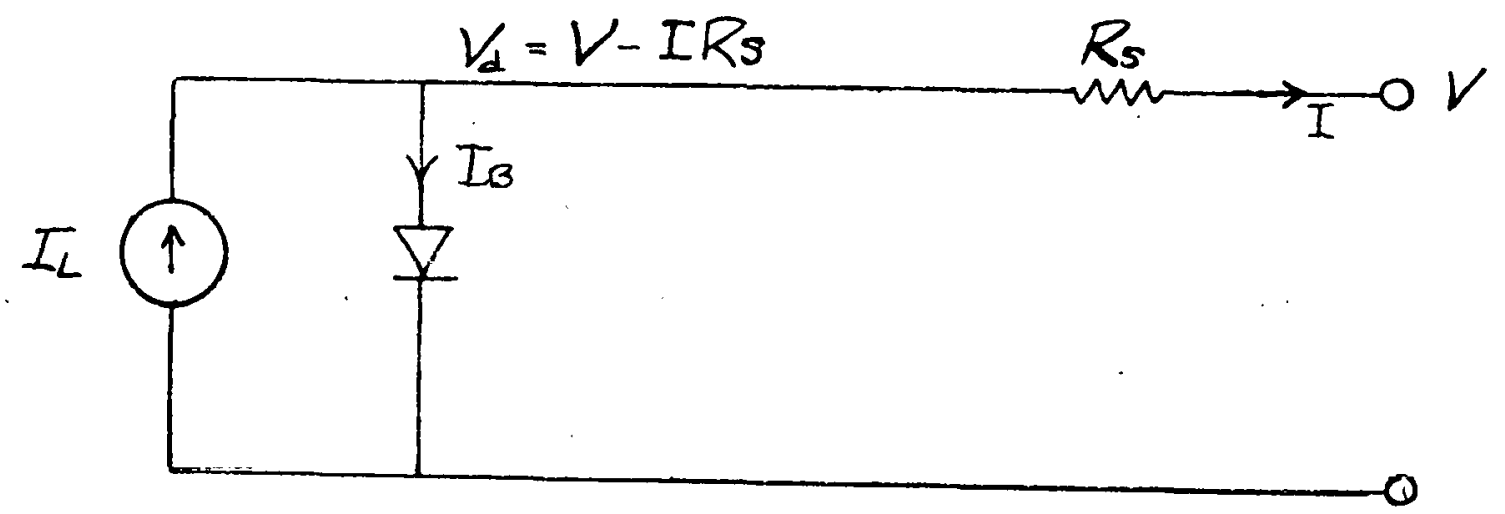

FIG. 13 Equivalent Circuit of a Diode with Series Resistance Only. 
Curve 692236-A

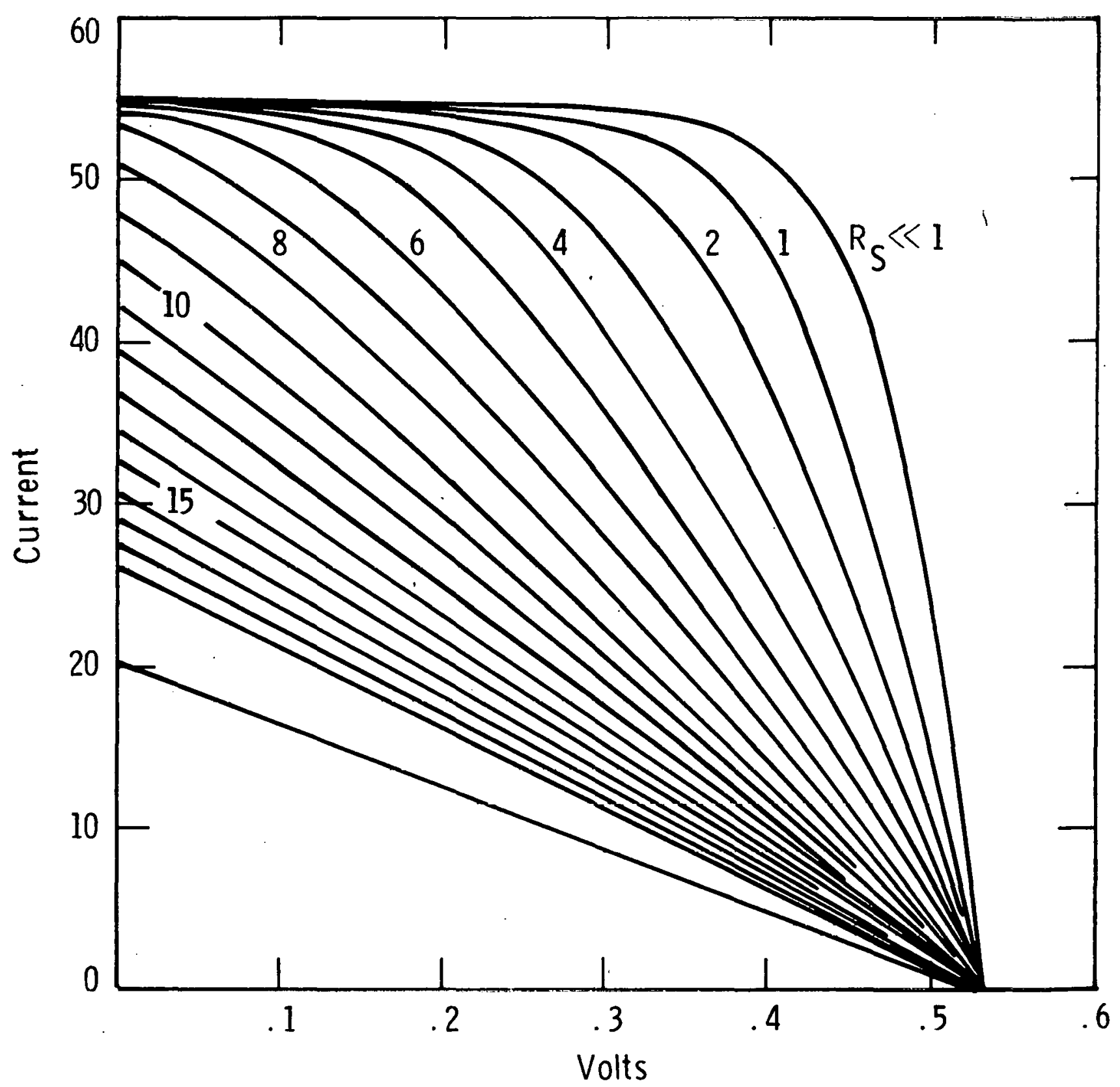

Fig. 14 The Effect of Sines Resistance on Solar Ce11 $\mathrm{I}-\mathrm{V}$ Characteristics. 


$$
\frac{\mathrm{d}}{\mathrm{dV}}(\log I)=\frac{\mathrm{q}}{\mathrm{kT}}\left(\cdot 1-\mathrm{R}_{\mathrm{s}} \frac{\mathrm{dI}}{\mathrm{dV}}\right)
$$

Let $\log I=u \quad \frac{d I}{d V}=e^{u} \quad \frac{d u}{d V}=I \quad \frac{d u}{d V}$

Substituting this in equation $(3.6 .16)$ gives

$$
\begin{aligned}
& \frac{d u}{d V}=\frac{q}{k T}\left(1-I R_{s} \frac{d u}{d V}\right) \\
& \frac{d u}{d V}\left(1+\frac{q I R s}{k T}\right)=\frac{q}{k T}
\end{aligned}
$$

which becomes

$$
\frac{d u}{d V}=\frac{d}{d V}(\log I)=\frac{a}{k T}\left(\frac{1}{1+\frac{q I R}{k T}}\right)
$$

Thus in the presence of $R_{s}$ the slope of $\log I$ vs $V$ will decrease with the increasing current resulting in a curvature of $l \circ g$ I vs $V$ plot in the current domain. If $R_{S}$ is very small ( $R_{S}<1 \Omega$ ) then curvature will not be visjble until high currents ( $I>20 \mathrm{ma}$ ), instead the $10 \mathrm{~g}$ vs $\mathrm{V}$ plot will appear to be a straight line with a slope $q / a k T$.

$$
\text { where } \eta=\frac{1}{1+q I \text { avR }} \frac{s}{k T} \quad>1
$$

For higher series resistances $\left(R_{S}>5 \Omega\right)$ the curvature is seen easily even at low currents ( $\mathrm{I}<\dot{10} \mathrm{ma}$ ).

Figure 15 shows data for samples which were dittused for two different times, $20 \mathrm{~min}\left(R_{S}=0.90 \Omega\right)$ and $480 \mathrm{~min}\left(R_{S}=.45 \Omega\right)$. Since $R_{S}$ is small the $\log I$ vs $V$ plot appears to be linear with slope = $1 / \mathrm{nkT}$ and $\mathrm{n}>1$. As predicted by equation $(3.6 .18)$ the slope of the 


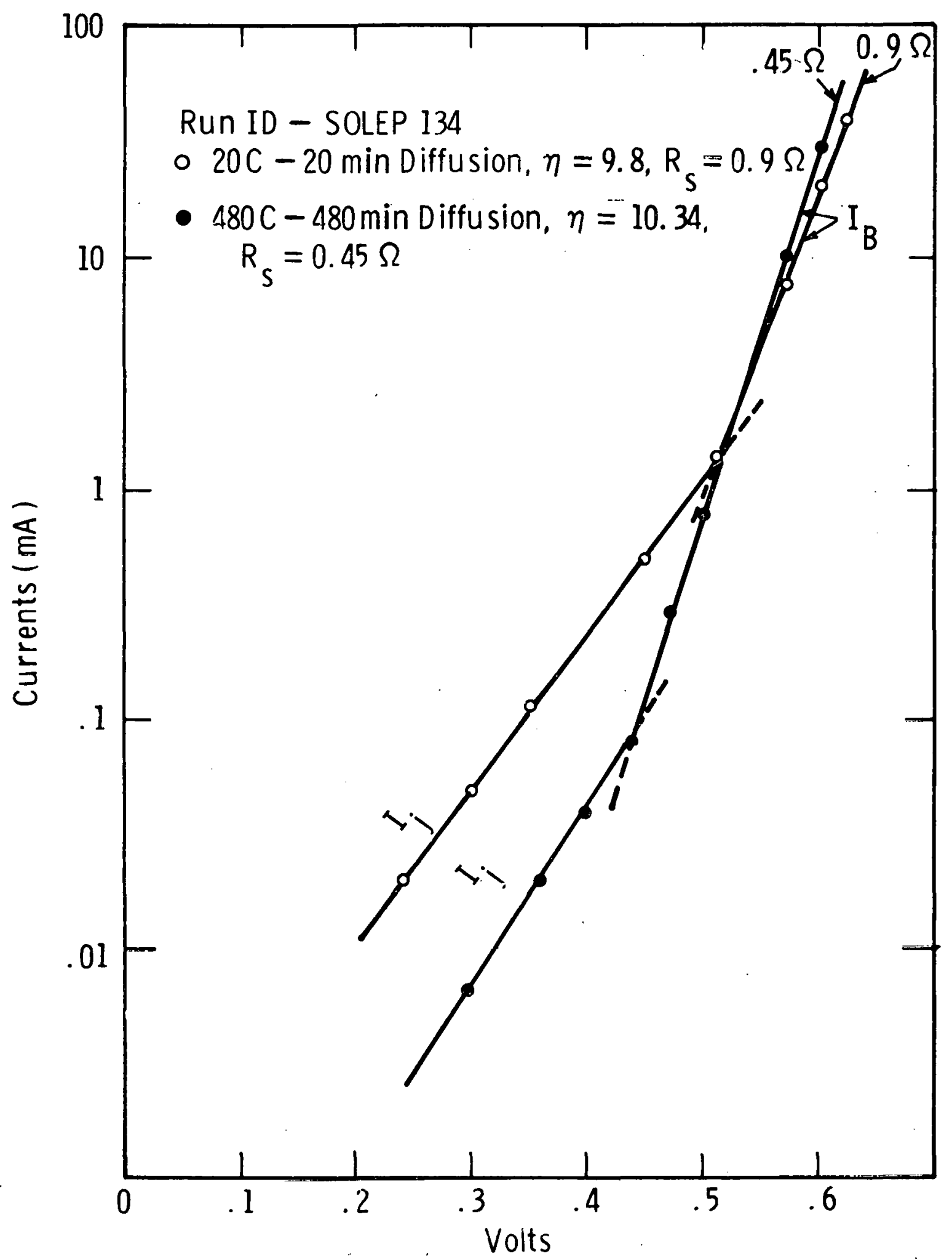

Fig. 15 Partially Transformed I-V Curves for the Samples Diffused 20 and 480 "inutes. The Effect of $R_{S}$ has not heen removed. 
$0.9 \Omega$ sample is smaller than $0.45 \Omega$ sample.

Series resistance can be experimentally determined by a combination of dark and lighted I-V curves. In the high current domain the measured $I-V$ data $c$ an be represented by the following equation

$$
\begin{aligned}
& I_{d}=I_{1}^{\prime}\left(e^{a\left(V-I_{d} R_{s}\right) / A k T}-1\right) \\
& \text {-dark I-V } \\
& I_{L}-I=I_{d}=\dot{I}_{I}\left\lfloor e^{q\left(V+I R_{S}\right) / A k T}-1\right) \\
& \text { - lighted I-V }
\end{aligned}
$$

where $A: 1$

For the same diode current $\left(I_{d}\right)$, the terminal voltage (V) will be different in dark and lighted casesbecause $V_{d}$ is same but the current (I) flows in opposite directions through $\mathrm{R}_{\mathrm{S}}$.

$$
\begin{aligned}
& \text { Id }=I_{i}\left(e^{q\left(V_{1}-I_{d} R_{s}\right) / A k T}-1\right)=I_{1} i_{1}\left(e^{q\left(V_{2}+\left(I_{2}-I_{d}\right) R_{s}\right) / k T}-1\right) \\
& \text { or } V_{1}-I_{d} R_{s}=V_{2}+\left(I_{L}-I_{d}\right) R_{s} \\
& \text { or } \Delta V=V_{1}-V_{2}=I_{i} R_{s} \\
& \text { or } R_{S}=\frac{\Delta V}{T_{L}}
\end{aligned}
$$

Thus a plot of $\log I_{d} v s ~ V$ in the dark and $\log \left(I_{L}-I\right)$ vs $V$ in the lighted case will show a parallel shift in the voltage (equal to $I_{L} R_{s}$ ) in the high current region. Figure 16 shows the experimental data on dark and lighted $I-V$ for a sample which was diffused for $20 \mathrm{~min}$. $\Delta V$ of $20 \mathrm{mv}$ was observed, while $I_{s c}$ was 22.2 ma and therefore $R_{s}=20 / 22.2=0.9 \Omega$.

Figure 16 indicates that a parallel shift is not observed in the low 


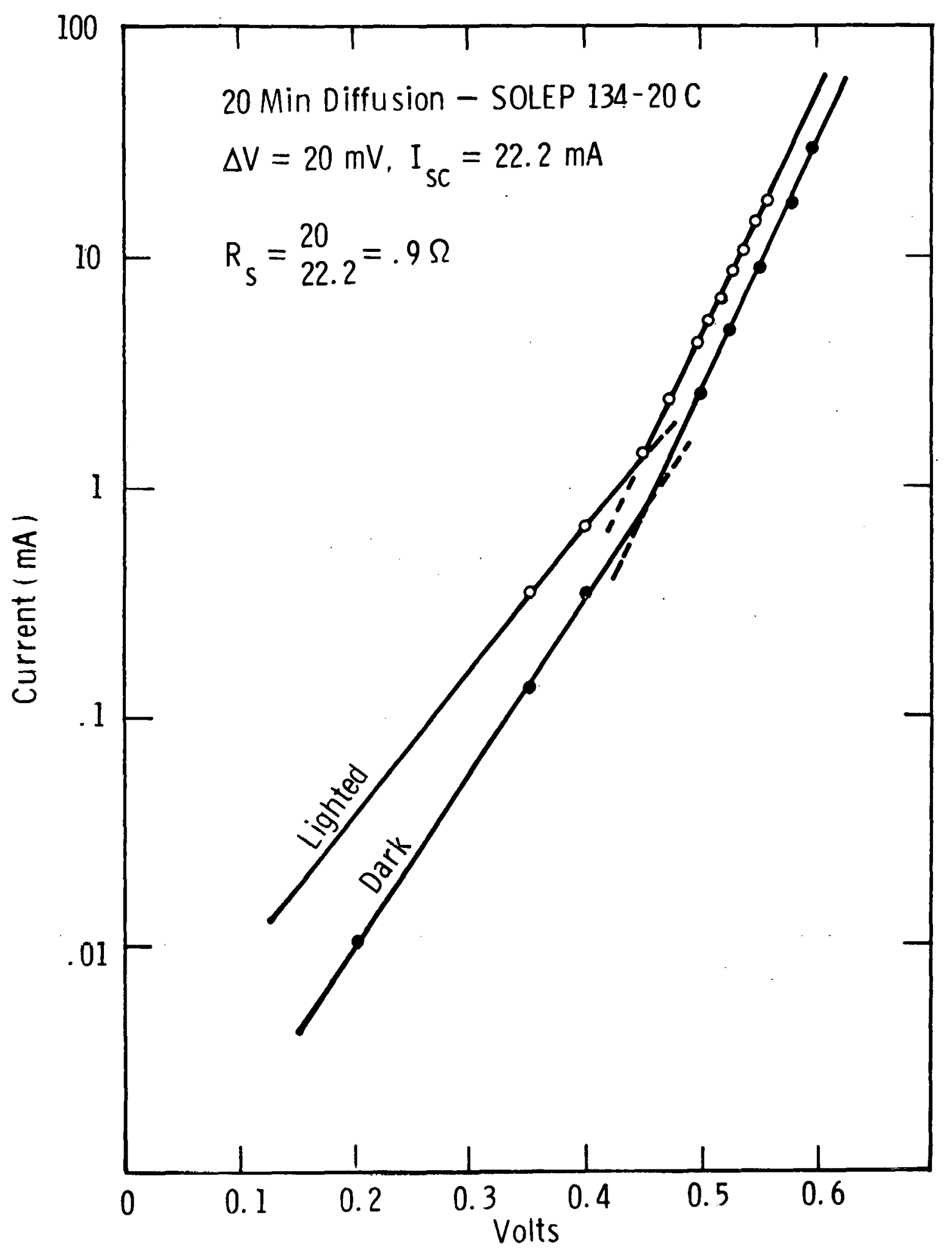

Fig. 16 The Measured Dark and Illuminated I-V Curves for the Sample Designated SOLEP 134-20C. 
current domain. The reason for this is not yet understood. However limited measurement accuracy in that range may be the explanation, especially in the lighted case where the difference of two large number $s\left(I_{d}=I_{L}-I\right)$ is taken to obtain a very small number. Also, the junction may respond somewhat differently in the presence of light.

\section{JUNCTION EXCESS CIURRFNT}

\section{Efrect of Junction Execas Gurxcnt on Eolar Col1 Effinioney}

In a solar cell $I=I_{L}-I_{d}=I_{L}-I_{B}-I_{f}-I_{s h}$. Thus if $I_{f}$ is large enough to be appreciable compared to the diffusion current near the peak power point, then for a given voltage I will be smaller. This will move the peak power point inward and reduce the fill factor and cell efficiency. Since $I_{j}=I_{2}\left(e^{q V / n k T}-1\right)$, increased $I_{j}$ shows up either as an increase in $\mathrm{Io}_{2}$ or a decrease in $\mathrm{n} \mathrm{I}_{\mathrm{j}}$ behaves like a higher impedance diode compared to $I_{B}$ so Isc will not be affected by junction excess current. However Voc will be reduced if appreciable amount of $I_{L}$ is lost as junction excess current.

\section{Determination of Junction Excess Current}

$I_{j}$ is obtained by subtracting the contributions of $R_{s h}, R_{s}$, and $I_{B}$ from the measured $I-V$ curve in the low current domain. The procedure is described in detail in section 3.5.3.2, Figure $10 . I_{j}$ is usually much less than 1 ma in the low current domaln $(0.1<V<0.4$ volts $)$. In most good devices $R_{s}<1 \Omega$ so in the low current domain $\left(V-I R_{s} \simeq V\right.$ )an effect of $R_{s}$ can be ignored. Also the effect of Rsh can be neglected $\left(R_{\text {gh }} \geq 30 \mathrm{~K}\right)$ in most cases. Thus, $I_{j}$ can be obtained simply by subtracting $I_{B}$ from the measured $I-V$ curve. The intercept of $\log \left(I_{j}\right)$ vs $V$ at $V=0$ gives $I_{2}$ and the slope gives $n\left(n=\frac{9}{\text { slope } \cdot " k T}\right)$. 


\section{Sintering Studies}

The role of junction excess current on the cell performance can be seen from some sintering experiments we conducted using $\mathrm{Ti}-\mathrm{Pd}-\mathrm{Ag}$ contacts. The devices were examined before and after sintering at either $425^{\circ} \mathrm{C}$ or $550^{\circ} \mathrm{C}$ in $\mathrm{H}_{2}$ for 15 minutes. Sintering is usually done to improve the contract resistance and the reliability of the metallurgical bond between the metal and the silicon. In our studies sintering usually resulted in some loss of efficiency in the devices so we analysed the I-V curves to determine the cause of it. Table 17 shows the performance of 10 solar cells before and after sintering at $425^{\circ} \mathrm{C}$. Figure 17 shows that sintering primarily increases the junction excess current, hense reducing the cell efficiency. This was found true for all the samples whose performance deteriorated after sintering. Sintering did not affect $R_{S}$, $I_{0}$, or $\tau$ appreciably. The only systematic and significant variation was found in $I_{j} \cdot R_{s h}$ decreased at times, but was found to be greater than $30 \mathrm{~K}$ in all cases after sintering. However, if the contract resistance is initially high $\left(\mathrm{R}_{S}>5 \Omega\right)$ then sintering lowered the series resistance and improved the cell efficiency, Figure 18.

\section{Junction Depth Experiments}

In our standard solar cell process diffusion time is 50 minutes which gives approximately a $0.3 \mu \mathrm{m}$ deep junction. In order to examine the effect of junction depth on cell performance and to further understand the possible adverse effects of sintering, a run was made with diffusion times of $20,40,80,100,320$ and 480 minutes. This should give junction depths in the range of 0.2 to 1 micron. The solar cell response of these samples is shown in Table 18. Contrary to conventional wisdom, no systematic variation in the cell efficiency with the junction depth was observed, instead average efficiency was about $9.9 \%$ in all the cases. This suggests that somehow the increase in sheet resistance is. balanced by the decrease in dead layer in shallow functions or vice versa for the deeper junctions. Our light source is somewhat rich in infrared which could be partially responsible for the lack of significant 
TABLE 17

RESULTS FROM SINTERING EXPERIMENT

RUN ID SOLEP 133 DIFFUSION TIME $=50 \mathrm{MIN}$.

\begin{tabular}{|c|c|c|c|c|c|}
\hline $\begin{array}{l}\text { SAMPLE } \\
\text { NUMBER } \\
\end{array}$ & $\begin{array}{l}\text { SAIPLE } \\
\text { STATE }\end{array}$ & $\begin{array}{l}\text { I sc } \\
m A\end{array}$ & $\begin{array}{l}\text { Voc } \\
\text { VOLTS }\end{array}$ & EFFICIENCY & \\
\hline 1 & Not sintered & 21.3 & .570 & 9.49 & . \\
\hline 1 & 3inteled & 20.9 & .563 & 8.85 & \\
\hline 2 & Not sintered & 21.7 & .565 & 8.81 & \\
\hline 2 & Sintered & 21.3 & .569 & 9.55 & \\
\hline 3 & Not sintered & 21.7 & .574 & 9.83 & \\
\hline 3 & Sintered & 21.2 & .571 & 9.65 & \\
\hline 4 & Not sintered & 22.4 & .573 & 9.67 & \\
\hline 4 & Sintered & 21.6 & .571 & 9.62 & \\
\hline 5 & Not sintered & 21.1 & .572 & 9.27 & \\
\hline 5 & Sintered & 21.2 & .564 & 8.95 & \\
\hline 6 & Not sintered & 21.5 & .574 & 9.91 & \\
\hline 6 & Sintered & 21.3 & .570 & 9.63 & \\
\hline 7 & Not sintered & 21.7 & .572 & 9.37 & \\
\hline 7 & Sintered & 21.5 & .567 & 9.59 & \\
\hline 8 & Not sintered & 21.7 & .576 & 10.13 & \\
\hline 8 & Sintered & 21.5 & .567 & 9.26 & . \\
\hline 9 & Not sintered & 21.9 & .575 & 9.82 & \\
\hline 9 & Sintered & 21.5 & .570 & 9.78 & \\
\hline 10 & Not sintered & 21.7 & .576 & 10.09 & \\
\hline 10 & Sintered & 21.4 & .566 & 9.56 & \\
\hline
\end{tabular}




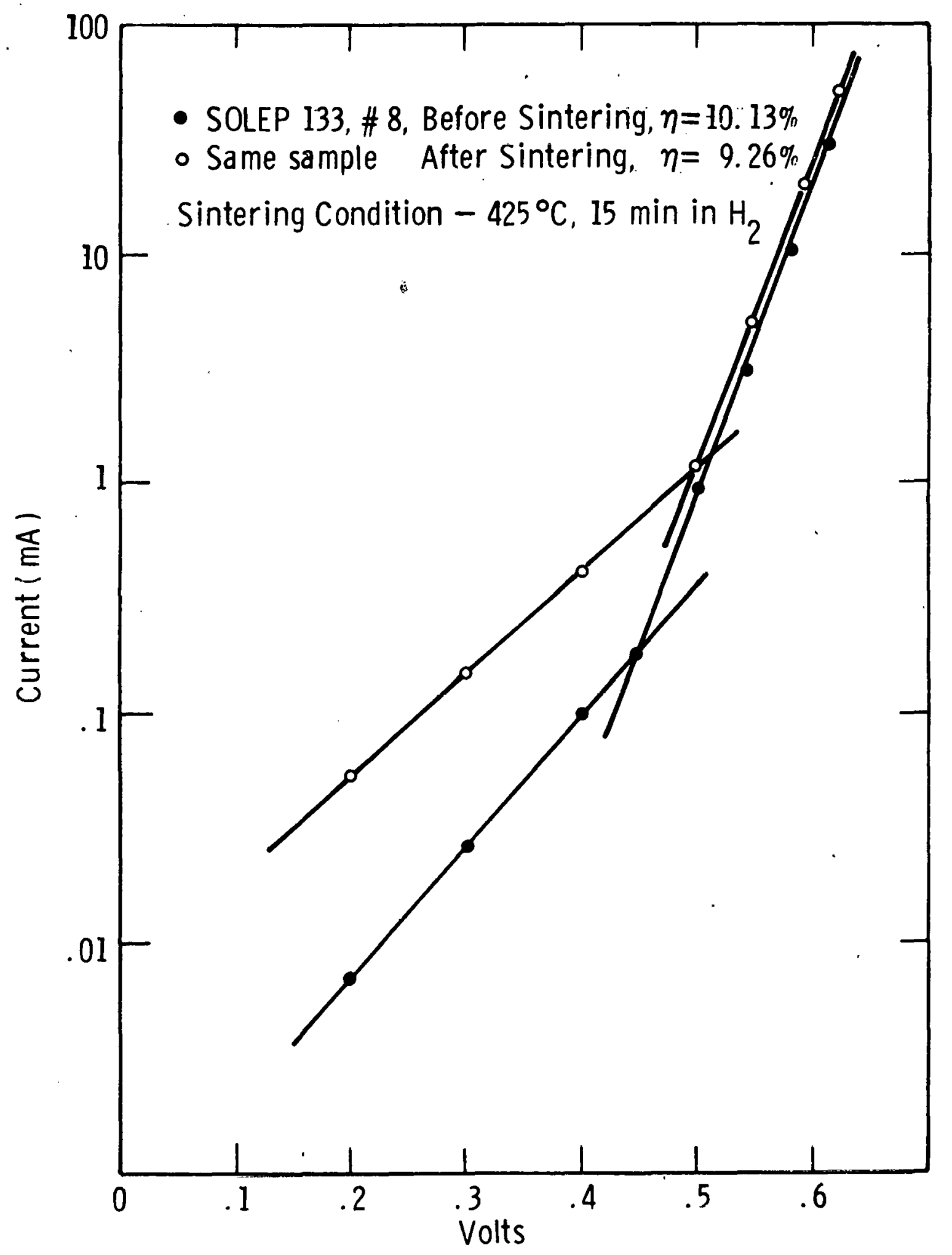

Fig. 17 Transformed Dark I-V Curves for the Sample SOLEP 133-8: - Before Sintering, o After Sintering at $425^{\circ} \mathrm{C}$. 


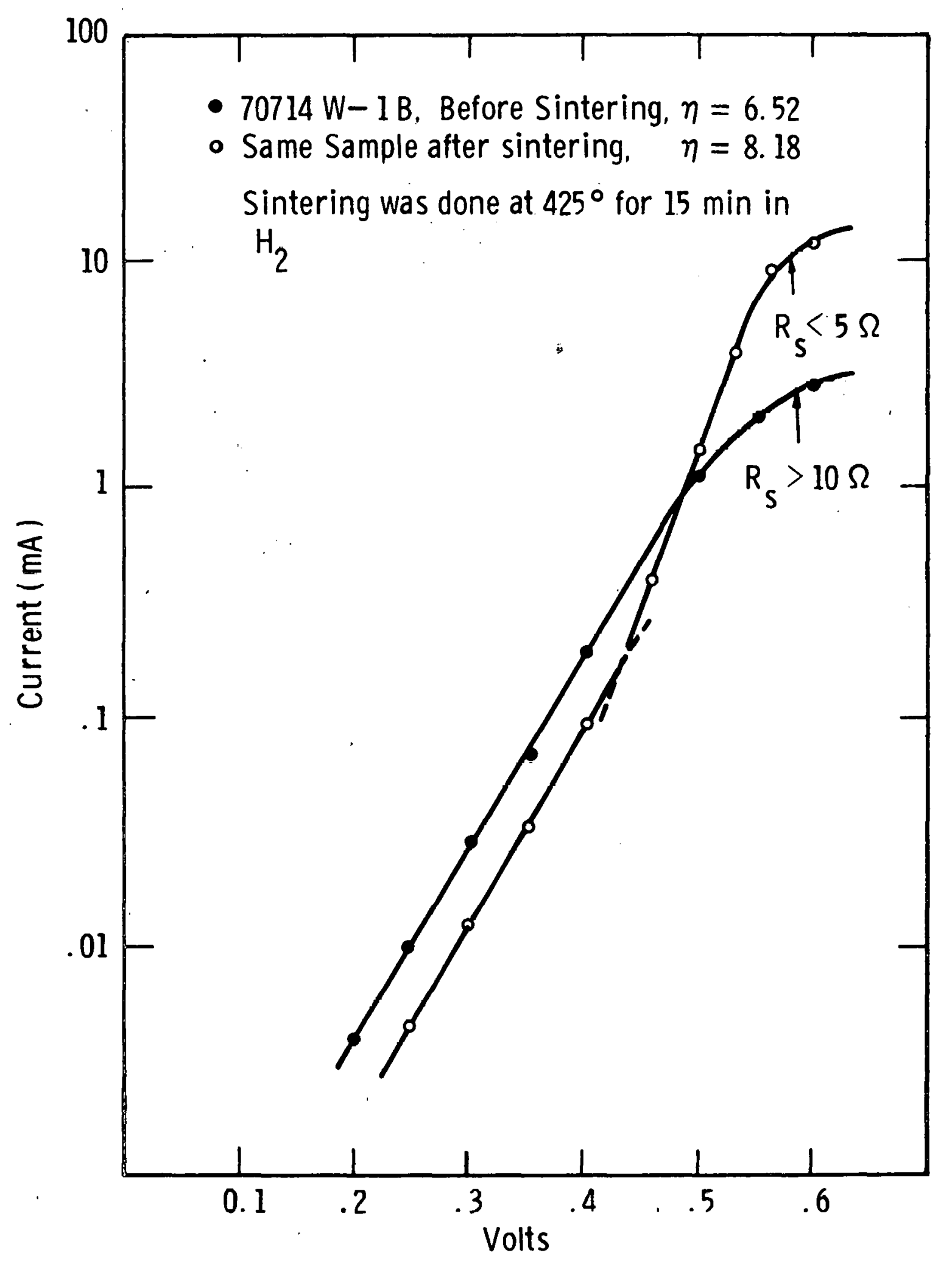

Fig. 18 Measured Dark I-V Curves for the Sample 70714W-13:

- Before Sintering, o After Sintering at $425^{\circ} \mathrm{C}$. 
TABLE 18 Effect of Diffusion Time (Junction Depth)

on Solar Cell Performance. AMI Illumination

No AR Coating.

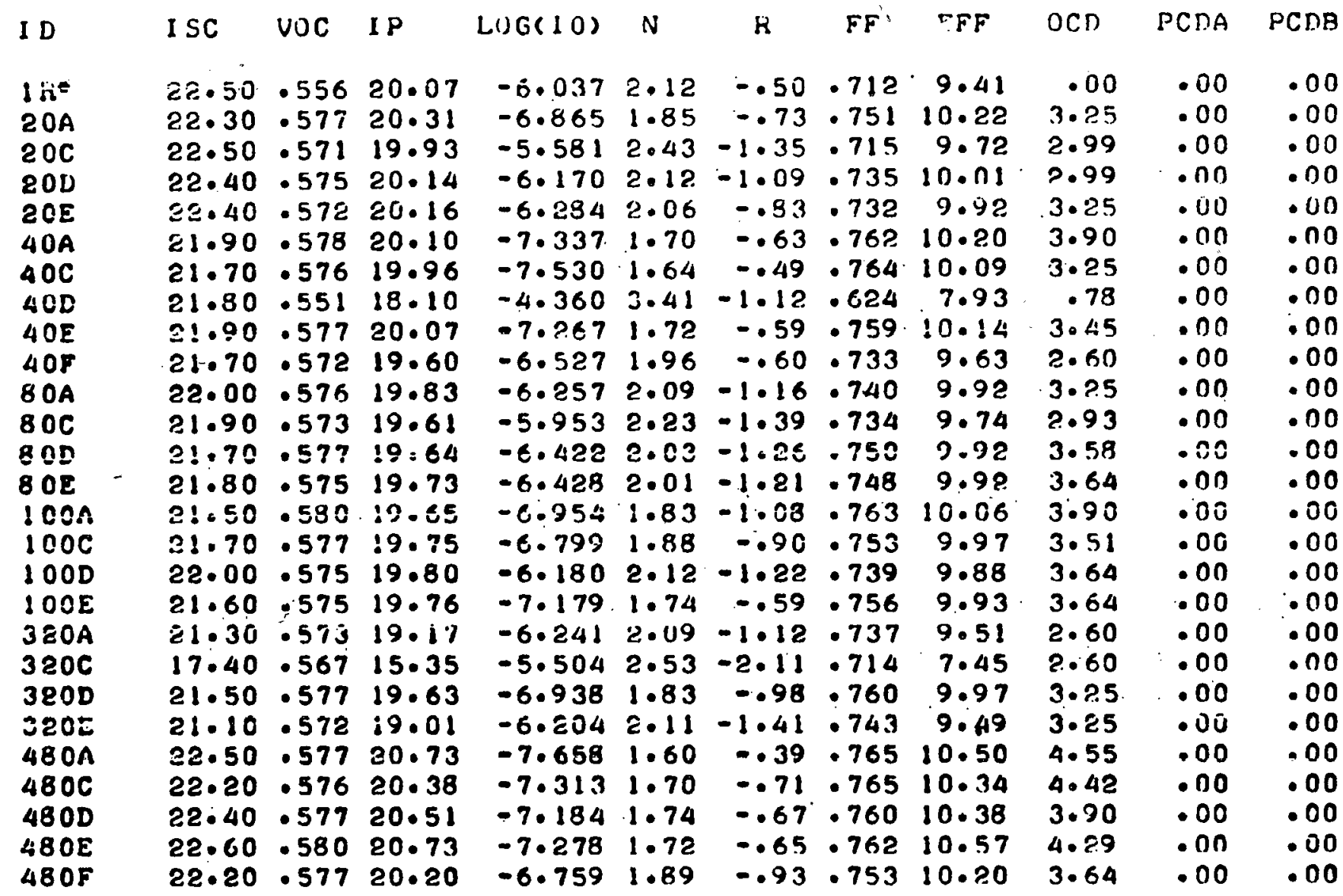

AVERAGES: 70816 BASELINE

NO DASELIINE

70816 MONSANTO LOT M Y 149 MULTI-DIFFUSION EXP

$\begin{array}{rrrrrrrrrr}.00 & .00\end{array}$ 
performance variation: Also the syton polished surfaces we used may have mitigated the adverse surface effects on shallow.junctions.

In another experiment samples with different junction depths were sintered, first at $425^{\circ} \mathrm{C}$ and then at $550^{\circ} \mathrm{C}$. Sintering at $425^{\circ} \mathrm{C}$ slightly degraded the cell efficiency in most cases. However, $550^{\circ} \mathrm{C}$ sintering affected the shallow junctions severely while deeper junctions were hardly affected. Results of this experiment are summarized in 'lable 19.: Figures 19 and 20 show a considerable increase in the. -junction excess current for the shallow junction compared to the deep junction devices. Thus deeper junctions, as expected, are found to be less sensitive to the sintering induced degradation. The reason for this degradation is quite clear, increased $I_{j}$. The physical mechanism by which degradation occurs though not fully understood, probably involves titanium diffusion, or migration into the junction region.

\section{LIFETIME}

\section{Effect of Lifetime on Solar Cell Efficiency}

Reduction in lifetime by processing or impurities diminishes the number of light generated carriers which survive to reach the junction. This produces a decrease of $I_{L}$ and thereby Isc. Reduction in lifetime increases Io and since 12,14

$$
V_{o c}=\frac{g}{k T} \ln \left(\frac{I_{L}}{I_{0}}+1\right)
$$

theretore Voc is also reduced. Both these effects reduce the solar cell efficiency. 
TABIE 19 Effect of Sintering Parameters on Solar Cell Performance.

\begin{tabular}{|c|c|c|c|c|c|}
\hline $\begin{array}{l}\text { SAMPLE } \\
\text { ID }\end{array}$ & $\begin{array}{l}\text { SINTERING } \\
\text { CONDITION }\end{array}$ & $\begin{array}{l}\mathrm{I}_{\mathrm{sc}} \\
\mathrm{mA}\end{array}$ & $\mathrm{V}_{\mathrm{OC}}$ & $\begin{array}{l}\text { OCD LIFETIME } \\
\tau(\mu \mathrm{sec})\end{array}$ & $\begin{array}{c}\text { EFF ICIENCY } \\
n \\
\end{array}$ \\
\hline $20 \mathrm{~A}$ & Unsintered & 22.3 & .577 & 3.25 & 10.22 \\
\hline $20 \mathrm{~A}$ & $425^{\circ} \mathrm{C}, 15 \mathrm{~min}, \mathrm{H}_{2}$ & 22.1 & .570 & 3.25 & 9.89 \\
\hline $20 \mathrm{~A}$ & $550^{\circ} \mathrm{C}, 15 \mathrm{~min}, \mathrm{H}_{2}$ & 22.4 & .562 & 1.56 & 8.70 \\
\hline $40 \mathrm{~A}$ & Unsintered & 21.9 & .578 & 3.9 & 10.20 \\
\hline $40 \mathrm{~A}$ & $425^{\circ} \mathrm{C}, 15 \min , \mathrm{H}_{2}$ & 21.6 & .571 & 3.9 & 9.99 \\
\hline $40 \mathrm{~A}$ & $550^{\circ} \mathrm{C}, 15 \mathrm{~min}, \mathrm{H}_{2}$ & 21.4 & .551 & .26 & 6.67 \\
\hline $80 \mathrm{~A}$ & Unsintered & 22.0 & .576 & 3.25 & 9.92 \\
\hline $80 \mathrm{~A}$ & $425^{\circ} \mathrm{C}, 15 \min , \mathrm{H}_{2}$ & 21.6 & .566 & 3.90 & 9.62 \\
\hline $80 \mathrm{~A}$ & $550^{\circ} \mathrm{C}, 15 \mathrm{~min}, \mathrm{H}_{2}$ & 21.6 & .563 & 1.17 & 7.81 \\
\hline $100 \mathrm{~A}$ & Unsintered & 21.5 & .575 & 3.9 & 10.06 \\
\hline $100 \mathrm{~A}$ & $425^{\circ} \mathrm{C}, 15 \mathrm{~min}, \mathrm{H}_{2}$ & 21.4 & .572 & 4.29 & 10.01 \\
\hline $100 \mathrm{~A}$ & $550^{\circ} \mathrm{C}, 15 \mathrm{~min}, \mathrm{H}_{2}$ & 21.2 & .562 & 1.04 & 7.60 \\
\hline $320 \mathrm{D}$ & Unsintered & 21.5 & .577 & 3.25 & 9.97 \\
\hline $320 \mathrm{D}$ & $425^{\circ} \mathrm{C}, 15$ min, $\mathrm{H}_{2}$ & 21.2 & .572 & 3.9 & 9.59 \\
\hline $320 \mathrm{D}$ & $550^{\circ} \mathrm{C}, 15 \mathrm{~min}, \mathrm{H}_{2}$ & 21.2 & .574 & 2.60 & 9.32 \\
\hline $480 \mathrm{~F}$ & Unsintered & 22.2 & .577 & 3.64 & 10.20 \\
\hline $480 \mathrm{~F}$ & $425^{\circ} \mathrm{C}, 15 \mathrm{~min}, \mathrm{H}_{2}$ & 21.7 & .575 & 4.55 & 10.21 \\
\hline $480 \mathrm{~F}$ & $550^{\circ} \mathrm{C}, 15 \mathrm{~min}, \mathrm{H}_{2}$ & 21.7 & .575 & 2.99 & 9.78 \\
\hline
\end{tabular}




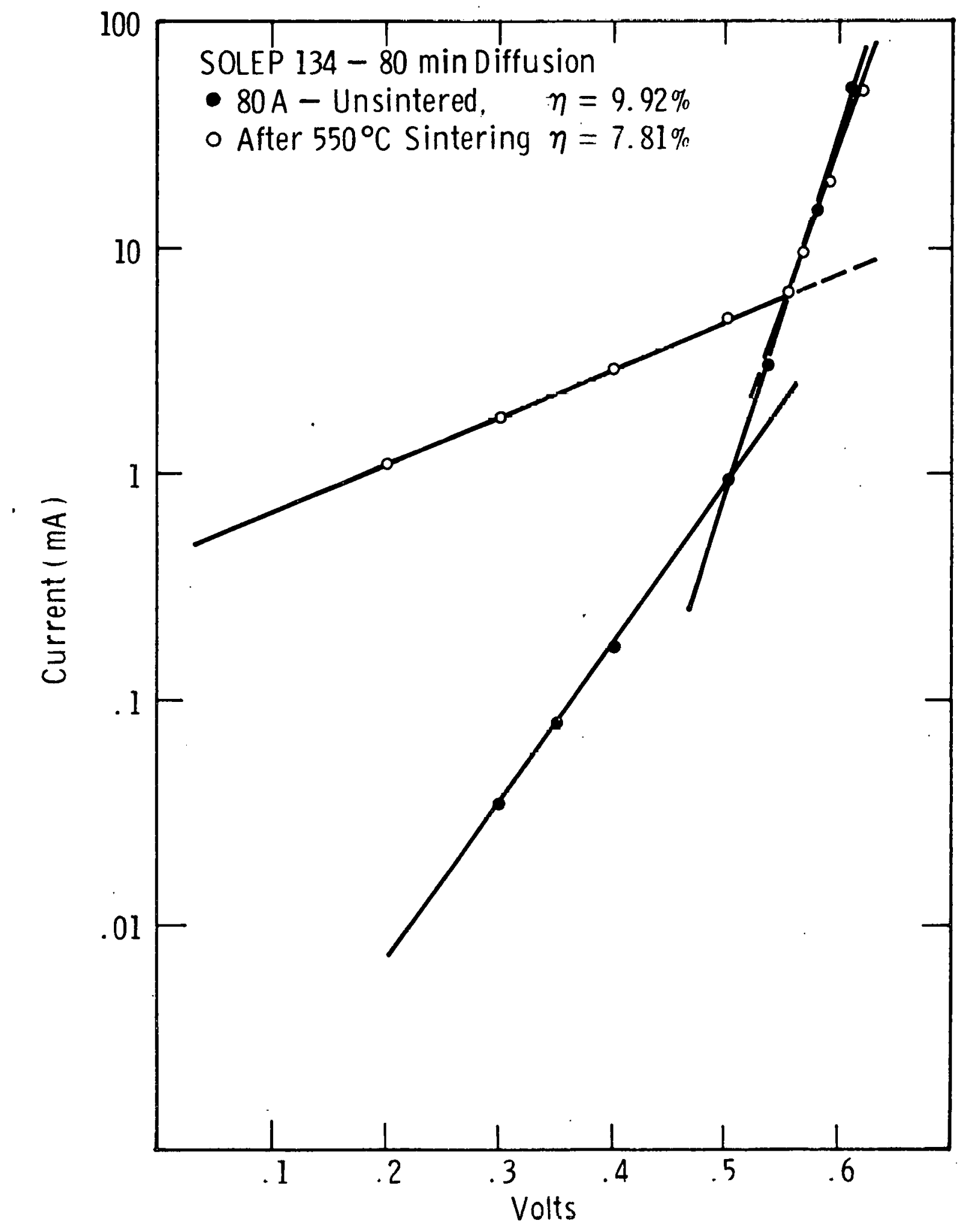

Fig. 19 Transformed Dark I-V Curves for the Sample SOLEP 134-80A: - Before, $\circ$ After Sintering at $550^{\circ} \mathrm{C}$. 


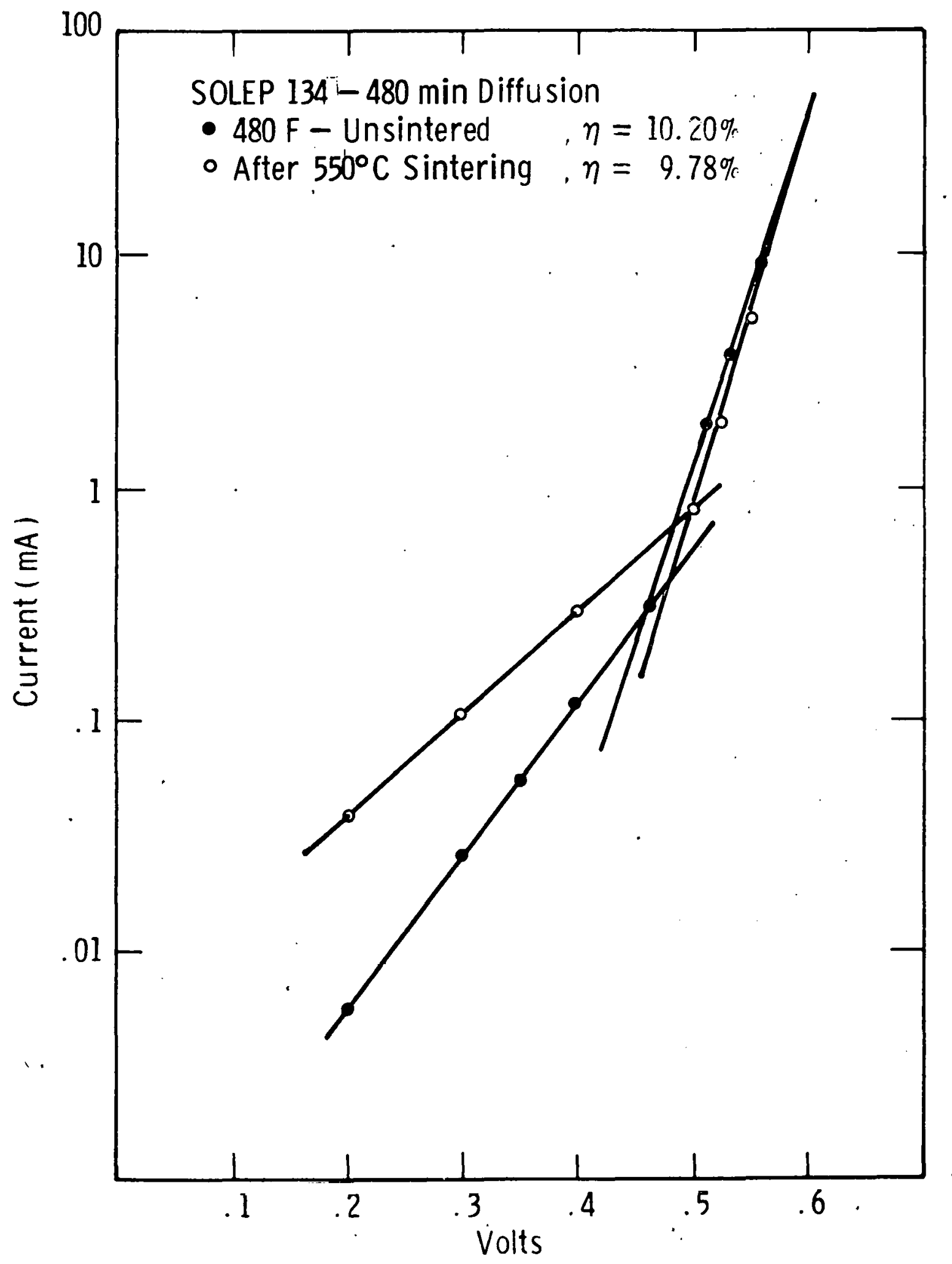

Fig. 20 Transformed Dark I-V Curves for the Sample SOLEP 134-480F: - Before, o After Sintering at $550^{\circ} \mathrm{C}$. 


\section{Determination of Lifetime from I-V Data}

Reduction in lifetime increases Io which mainly effects the bulk diffusion current.

$$
\begin{aligned}
& I_{B}=I_{0}\left(e^{q V / k T}-I\right) \\
& \text { or: } \quad \log I_{B}=\log I_{0}+q V / k T \text { in the high current domain }
\end{aligned}
$$

For a fixed $I_{D}$

$$
\Delta V=-\frac{k T}{q} \Delta\left(\log I_{0}\right)=\text { constant }
$$

This reduction in lifetime will move $I_{B}$ parallel and, to the left in Figure 21.

After subtracting the contributions of $I_{j}$ and Ish from the measured $I-V$ data in the high current segment one gets

$$
\begin{gathered}
I=I_{0}\left(e^{Y\left(V-I R_{s}\right) / k T} \cdot-1\right) \\
\text { cr } \log I=\log I_{0}+\frac{V}{2.3 k T}\left(V-I R_{s}\right)
\end{gathered}
$$

If $R_{S}$ is known then any point (V, I) can be used to get $I_{0}$. However if $R_{3}$ is not known a point at lower current ( $~ 1$ ma) should be used so that $V-I R_{3}=V$. For example, let us determine the lifetime of the baseline sample $\left(\left[\pi_{i}\right]=0\right)$ in Figure 21 by using a point ( $1 \mathrm{ma}, \cdot 48$ v) from $I_{B}$.

$$
\log 1=\log I_{0}+\frac{1}{2.3 \times 25}
$$


$I R_{S}$ is neglected because $V-I R_{S}=480-1 \simeq 480$

$$
\begin{aligned}
\log I_{0} & =8.347 \\
\text { or } \quad I_{0} & =4.49 \times 10^{-9} \mathrm{ma}
\end{aligned}
$$

$I_{0} \approx \frac{A q n i^{2}}{N_{A}} \sqrt{\frac{D_{n}}{\pi_{n}}} \quad 3.6 .25$ for $N^{+} P$ device

$$
\mathrm{N}_{\mathrm{A}}=6 \times 10^{15} \mathrm{~A}=1 \mathrm{~cm}^{2}, \mathrm{D}_{\mathrm{n}}=25
$$

$$
I_{0}=\frac{1.3 .33 \times 10^{-15}}{\sqrt{T}}
$$




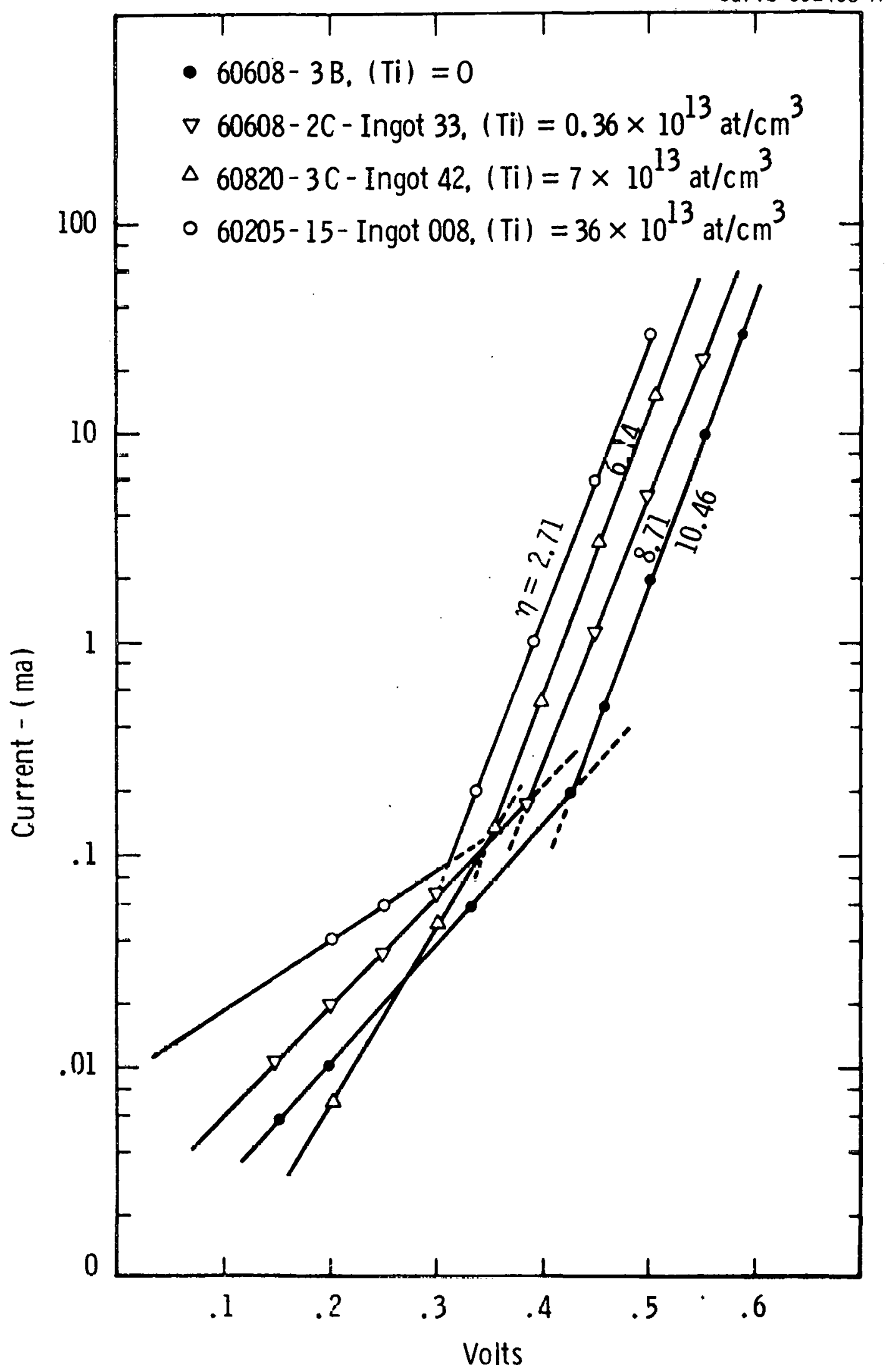

Fig. 21 Transformed Dark I-V Curves for Titanium-doped 4 sicm Silicon. 


$$
\begin{aligned}
& \text { or } \sqrt{\tau}=\frac{13.33 \times 10^{-15}}{4.49 \times 10^{-12}}=2.969 \times 10^{-3} \\
& \text { or } \tau=8.8 \mathrm{usec} .
\end{aligned}
$$

The measured OCD lifetime on this sample was $4.55 \mu \mathrm{sec}$ which is somewhat smaller because of surface recombination at the back surface which is not taken into account in equation (3.6.25). Test devices fabricated with back surface fields actually show OCD lifetime of $\simeq 9-10$ usec.

\section{Observations for Ti-doped Solar Cells}

The data for Ti-doped silicon, Table 20, indicate how the technique can be applied to evaluate impurity effects. Here, solar cell efficiency is significantly and systematically reduced with the increase in $\mathrm{T} 1$ concentration in the substrate. Analysis of $I-V$ data Figure 21 shows that $\mathrm{T} 1$ addition does not significantly alter $R_{s h}, R_{s}$, or $I_{j}$ to account for the degradation. However, a systematic and significant increase in $I_{0}$ is observed with the addition of $\mathrm{Ti}$ Figure 21 . Thus, here the impurity addition primarily reduces the bulk lifetime, confirming a conclusion drawn from the impurity effect modeling as discussed in the previous quarterly reports. 1,3 This is not necessarily the mechanism of cell degradation for all impurities viz, Copper. 
Table 20

Effect of Titanlum Concencration

on Solar Cell Parameters

\begin{tabular}{|c|c|c|c|c|c|c|}
\hline RUN ID & $\begin{array}{l}\text { SAMPLE } \\
\text { ID }\end{array}$ & $\begin{array}{l}\mathrm{Ti} \text { CONC. } \\
\text { atoms } / \mathrm{cm}^{3}\end{array}$ & $I_{s c}$ & $\mathrm{~V}_{\mathrm{oc}}$ & $\begin{array}{l}\text { OCD } \\
\text { Lifetime. }\end{array}$ & $\begin{array}{c}\text { Efficiency } \\
n\end{array}$ \\
\hline 60608 & $3 B$ & 0 & 22.5 & .555 & 4.55 & $10.46 \%$ \\
\hline 60608 & $2 \mathrm{C}$ & $0.36 \times 10^{13}$ & 20.2 & .544 & 1.69 & $8.71 \%$ \\
\hline 60820 & 1S & $7 \times 10^{13}$ & 16.6 & .494 & .26 & $5.97 \%$ \\
\hline 60205 & 1S & $36 \times 10^{13}$ & 7.80 &. .445 & .07 & $2.71 \%$ \\
\hline
\end{tabular}




\subsubsection{Summary of I-V Curve Analysis Studies}

The measurement and characterization technique described above has provided us with the ability to separate and analyze various process and impurity dependent mechanisms which influence soliar cell performance. We can now quantitatively measure the effect of impurities and or processes on the junction region as compared to their effect on base region. It is also possible to distinguish impurity induced effects on the control interface resistance should such occur.

On-going experiments are being conducted to examine the behavior of impurity contaminated cells. The data obtained for titanium shows the degradation to be primarily the result of lifetime loss in the base as had been deduced from the impurity modeling analysis. Of particular interest are cells containing iron, nickel or copper since, in the impurity model, these metals seem to display a non-linear effect on lifetime. It is conjectured that this may be a consequence of precipitate formation, known to occur for these metals and which are expected to affect junction properties more strongly than base properties. 


\section{CONCLUSIONS}

$\mathrm{P}^{+} \mathrm{N}$ solar cells appear to be more resistant to the harmful effects of $\mathrm{Ti}$ and $\mathrm{V}$ than do conventional p-base devices. For example, at comparable vanadium contamination levels $\left(4 \times 10^{14}\right.$ at $\left.\mathrm{cm}^{-3}\right) \mathrm{n}$-base devices exhibit efficiencies about $70 \%$ of the uncontaminated baseline cells while p-base devices are only $40 \%$ as efficient. Nickel, on the other hand, degrades the cell performance of $n$-base devices to about $54 \%$ of the baseline value while having little effect on $\mathrm{N}^{+} / \mathrm{P}$ cells. Lifetime reduction seems to be the primary mechan1sm by which $\mathrm{T} 1$ and $\mathrm{V}$ reduce cell performance. Nickel, however, influences solar cell output through changes in junction properties. The detailed influence of nickel will be examined shortly by a newly developed quantitative I-V technique which facilitates separation of impurity effects in the base and junction regions of the device.

In $4 \Omega \mathrm{cm}$ p-base material molybdenum degrades cell performance to about $75 \%$ of the baseline value when the metal content is about $6 \times 10^{14}$ at $\mathrm{cm}^{-3}$. Phosphorus at a similar concentration has essentially no effect on efficiency. The effect of high compensation levels will be examined in future work. Solar cells fabricated on silicon containing twins and grain boundaries introduced purposely (grain sizes up to about $1 \mathrm{~mm}$ : exnibit about $70 \%$ the efficiency of the single crystal baseline material. This metal-free polycrystalline material will be used for comparative studies with polycrystalline ingots containing selected impurities. 


\section{PROGRAM STATUS}

\subsection{Present Status}

The program is essentially on schedule, Figure 22, although chemical analysis of the ingots lags because of downtime on the mass spectrometer.

During this quarter we have:

- Completed the growth of thirteen nev ingots; all first generation n-base ingots and half the first generation low resistivity crystals are grown.

- Completed growth of ingots containing the new impurities $C, \mathrm{Ca}$, and $\mathrm{P}$ as secondary dopants in $4 \Omega \mathrm{cm}$-type silicor.

- Completed solar cell evaluation of the first ingots containing Mo and $\mathrm{P}$ and also evaluated baseline polycrystalline material for grain boundary/impurity studies.

- Calibrated the laser-excited PCD equinment and determined the conditions for precision recombination lifetime measurements.

- Initiated the study of processing effects on solar cell performance.

- Developed and tested a quantitative method for disecting the effect of impurities and processing on the base, junction, and contact behavior in contaminated solar cells.

\subsection{Future Activity}

During the next quarter all first generation low resistivity ingots will be grown and characterized as will most second generation $\mathrm{n}$-base ingots. Polycrystalline metal-doped ingots will be prepared. The variation of impurtty concentration and cell performance as a function of growth rate will be investigated. Processing studies will be extended to encompass impurity-bearing silicon. The solar cell performance of all first generation $n$-base ingots will be measured and the evaluation of 


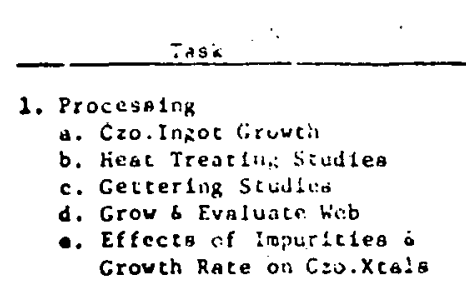

2. Increased Acceptor Doping - Grar lov a bacelling i. G:ua twpuide-Dupud Ingoce c. Retabiloh Std.Procesa d. Evaluate impurity Effecto

3. H-Base Cello

a. Crow Barel ine Ingots

b. Eatabliah call Proceas

c. Grow lietal-Doped Ingoce

d. Evaluete Metal-Dopad Ingoto o Wafero

6. Pxtend Present Impurity Mateix

a. Grow Raseline Ingoto

b. Grou Single 6 MultiplyDoped Irgoto

c. Evaluate Iingota o Callo

5. Study Crain Boundary/Impurity Diatribistion Effecto

i. Grov Bastilne inzota

b. Srow Metal-Dbóa ingoen

c. Evalunte Vaters \& Collu

6. $P_{2} / C_{20}$. Comparioon

a Crou Fz Baeiline Lngotá

b. Cirow Metul-Doped Fz Ingote

c. Evalunte Ingote c Cello

7. Daca Refinewent, Aanyeiso

b Synthesia

8. Pejorta
a. Frogram P!an
b. Monthly
c. Quarterly
d. Deafe Finel
e. PInal

9. Meetinge

e. l'ask litedgzation

b. Workahop

c. Cuntector Rev/uTh

20. Dellverabie Iteme

a. Call Blanico

b. Lab Notebooke

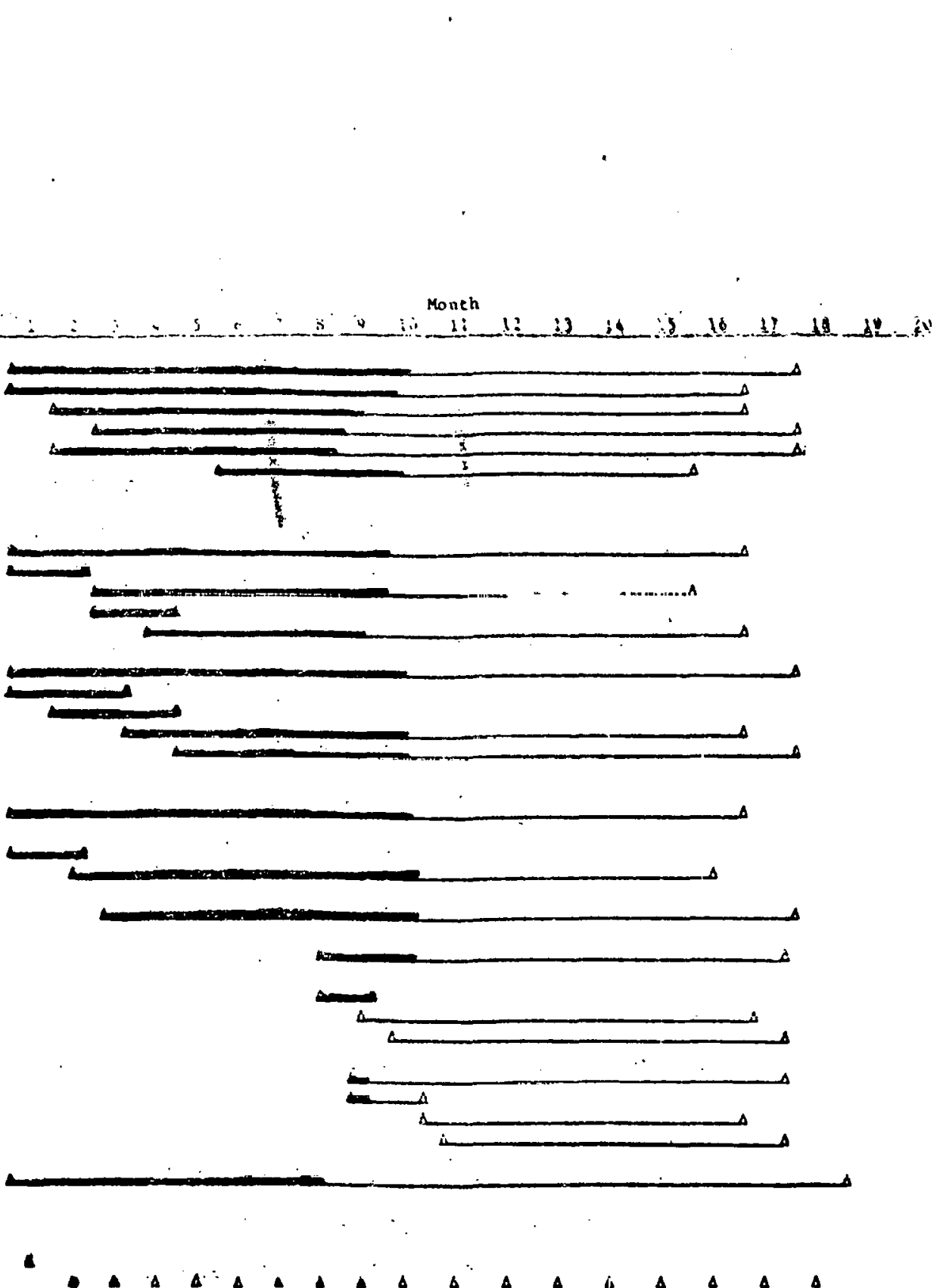

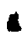
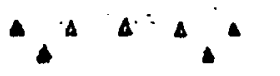

$\Delta \Delta$

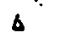

$a^{A}$

b

$\Delta \Delta \Delta \quad \Delta$

4
4
4

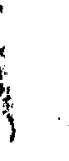

a

$\mathbf{A}$

$\therefore$

- an reciurad -

FIG. 22 Program Schedule 
about half the first generation low resistivity ingots will also be finished. Detailed I-V measurements will be carried out to determine the way in which impurities like $\mathrm{Cu}, \mathrm{Fe}$, and $\mathrm{Ni}$ degrade device performance. 


\section{REFERENCES}

1. R. H. Hopkins, et. al., Fifth Quarterly Report, Silicon Materials Task (Part 2), ERDA/JPL-954331-77/1, December 1976.

2. H. W. Gutche, Final Report, Determination of a Definition of Solar Grade Silicon ERDA/JPL-954338-76.

3. R. H. Hopkins, et. al., Seventh Quarterly Report, Silicon Materials Task (Part 2), ERDA/JPL 95433-77/3, June 1977.

4. J. S. Blakemore and K. C. Nomura, "Influence of Transverse Modes on Photoconductive Decay in Filaments," J. Appl. Phys. 31, p 7531960.

5. R. H. Hopkins, et. al. Second Quarterly Keport, Silicion Mazerials Task (Part 2), ERDA/JPL-954331-76/2, May 1976, P. 38.

6. K. Graff, H. Pieper, G. Goldback, "Carrier Lifetime Doping of p-Type Silicon by Annealing Processes," Semiconductor Silicon/1973, edited by Huff and Burgess, Electrochem. Soc., 111 (1973).

7. W. Shockley, Be11 Syst. Tech. J., Vol. 28, p.p.435-489, July, 1949.

8. P. E. Gray, D. Dewilt, A. R. Boothroyd and J. F. Gibbons, Physical Electronics and Circuit Models of Transistors, SEEC Vo1. 2, John Wiley \& Sons, Inc.'N.Y.

9. C. T. Sah, R. N. Noyce anci N. Shockley, Proc. IR̈E, Vol. 45, PR. 17?81243, Sept. 1957.

10. F. A. Lindholm, A. Neugrosche1, C. T. Sah, M. P. Godlewski and H. W. Brandhorst, Ir., IEEE Transaction on Electron Devices, Vol. ED-24, No. 4, pp 402-410, Apri1 1977.

11. M. Wolf and H. Rauscheabach, "Solar Ce11s", edited by C. E. Backus, pp. 147-170, IEEE Press, N.Y. 1976.

12. M. Wolf, "Solar Cells" edited by C. E. Backus pp 191-201, IEEE Press, N.Y., 1976.

13. A. Neugroschel, F. A. Lindholm and C. T. Sah, IEEE Transactions on E1ectron Devices, Vol ED-24, No. 6, pp. 662-671, June 1977.

14. S. M. Sze, Physics of Semiconductor Devices, Wiley Interscience, N.Y., 1967. 
7. APPENDIX

BEST ESTIMATE OF IMPURITY CONCENTRATION

IN ALL INGOTS PRODUCED TO DATE 
Best Estimate of Impurity Conc. $\left(10.15\right.$ atoms $\left./ \mathrm{cm}^{3}\right)$

$$
\text { W }
$$$$
006-119-001 / 1202
$$$$
\text { W-007-Cu-001/1294 }
$$$$
\text { W-008-Ti-001/1298 }
$$$$
\text { W-009-V-001/12100 }
$$$$
\text { W-010-Ni-002/12101 }
$$$$
W-011-2 r-001 / 12105
$$$$
\text { W-013-Mn-002-1287 }
$$$$
\text { W-014-00-000/12103 }
$$$$
\text { W-U1b-2n-UU1/12108 }
$$$$
\text { W-017-Cu-003/1295 }
$$$$
\mathrm{W}-018-\mathrm{Fe}-002 / 12114
$$$$
\mathrm{W}-019-\mathrm{Cu}-003 / 1293
$$$$
\mathrm{W}-020-00-000 / 1289
$$$$
\text { W-021-Kg-001/12116 }
$$$$
W-022-00-000 / 12109
$$$$
w-023-00-000 / .22122
$$$$
\text { iv-024-Mg-002/12117 }
$$$$
\text { wi-025-00-000/12120 }
$$$$
\mathrm{N}=026-\mathrm{Y} / \mathrm{n}-003 / 12123
$$$$
\mathrm{W}-027-\mathrm{Mn} / \mathrm{Cu}-001 / 12125
$$

(1) $030-\mathrm{Cr} / \mathrm{Cu}-001 / 12129$ 
Ingot

Identification

$$
\begin{aligned}
& \text { W-031-Cr/Mn-001/12130 } \\
& \mathrm{W}-032-\mathrm{Mg}-003 / 12132 \\
& \text { W-033-Ti-002/12135 } \\
& \text { W-034-00-000/12137 } \\
& \text { W-035-V-002/12138 } \\
& \mathrm{W}-036-2 \mathrm{r}-002 / 12145 \\
& \text { W-037-Zr/Ti-001/12146 } \\
& \text { W-038-Al-002/01-22 } \\
& \text { W-039-Ni-003/12147 } \\
& \mathrm{W}-040-\mathrm{Cr} / \mathrm{Ni}-001 / 12151 \\
& \mathrm{~W}-041-\mathrm{Ni} / \mathrm{Cr} / \mathrm{Cu}-001 / 12153 \\
& \text { W-042-Ti-003/1297 } \\
& \mathrm{W}-043-\mathrm{Fe} / \mathrm{Ti}-001 / 12158 \\
& \mathrm{~W}-044-\mathrm{Fe}-003 / 12156 \\
& \text { W-045-Cr/Fe/Ti-001/12159 } \\
& \mathrm{W}-046-\mathrm{Fe} / \mathrm{V}-001 / 12160 \\
& \mathrm{~W}-047-\mathrm{Cu} / \mathrm{Ni} / \mathrm{Zr}-001 / 12165 \\
& \text { W-048-Ti-004/12167 } \\
& W-049-V-003 / 12168 \\
& \text { W-050-Ti/V-001/12169 } \\
& \text { W-051-Cu/Ti-001/12170 } \\
& \text { W-052-Ni-004/01-36 } \\
& \text { W-053-Poly/12160 } \\
& W=054-00-000 / 12182 \\
& \mathrm{~W}-055-\mathrm{Cu}-004 / 12189 \\
& \mathrm{~W}-056-\mathrm{Cu}-005 / 12190 \\
& \text { [V-057-00-000/12184 } \\
& \text { W-058-00-000/12188 } \\
& \text { W-059-00-000/12198 } \\
& \mathrm{h}-050-00-000 / 12202 \\
& \text { W-06I-Cr/Ti-001/12191 } \\
& \mathrm{W}-062-\mathrm{N}-\mathrm{Cu}-001 / 12203 \\
& \mathrm{~W}-063-\mathrm{N}-\mathrm{Cr}-001 / 12211
\end{aligned}
$$

\begin{tabular}{|c|c|}
\hline $1.0 / 1.3$ & $(1.0 / 2.5)$ \\
\hline 0.32 & $(0.32)$ \\
\hline 0.0036 & -- \\
\hline- & - \\
\hline 0.004 & - \\
\hline$<0.025$ & $(<0.025)$ \\
\hline$<0.015 / 0.40$ & $(0.015 / 0.36)$ \\
\hline 60 & (34) \\
\hline 8 & (4) \\
\hline $0.8 / 2.4$ & $(1.0 / 3.5)$ \\
\hline $2.4 / 0.8 / 1.7$ & $(3.0 / 1.7 / 2.3)$ \\
\hline 0.07 & -- \\
\hline $0.56 / 0.06$ & -- \\
\hline 0.017 & - \\
\hline $0.65 / 0.43 / 0.06$ & $(0.2 / 0.5 / 0.06)$ \\
\hline $0.57 / 0.07$ & -- \\
\hline $2.0 / 1.0 /<0.015$ & $(<2.4 /<1 /<1)$ \\
\hline 0.00036 & -- \\
\hline 0.0004 & - \\
\hline \multicolumn{2}{|c|}{$0.00036 / 0.0004$} \\
\hline $2.0 / 0.36$ & $(4 / 0.36)$ \\
\hline 4.5 & $(4.0)$ \\
\hline- & -- \\
\hline- & -- \\
\hline 0.05 & $(<1)$ \\
\hline 65 & $(70)$ \\
\hline-- & -- \\
\hline-- & - \\
\hline-- & -- \\
\hline-- & -- \\
\hline $1.0 / .02$ & $1.0 /<1$ \\
\hline 1.7 & $(2.0)$ \\
\hline 1.0 & $(1.0)$ \\
\hline
\end{tabular}

Best Estimate of Impurity Conc. (10 15 atoms $/ \mathrm{cm})$ 


$$
\begin{aligned}
& \mathrm{W}-064-\mathrm{N}-\mathrm{Mn}-001 / 1221.2 \\
& \mathrm{~W}-065-\mathrm{N}-\mathrm{Ti}-001 / 12218 \\
& \mathrm{~W}-066-\mathrm{Ti}-005 / 12222 \\
& \mathrm{~W}-067-\mathrm{Cr} / \mathrm{Mn} / \mathrm{Ti}-001 / 12229 \\
& \mathrm{~W}-068-\mathrm{Cr}-004 / 12228 \\
& \mathrm{~W}-069-\mathrm{Fe}-004 / 12232 \\
& \mathrm{~W}-070-\mathrm{Al}-003 / 12234 \\
& \mathrm{~W}-071-00-000 / 05-07 \\
& \mathrm{~W}-072-\mathrm{Cr}-005 / 12237 \\
& \mathrm{~W}-073-\mathrm{Cr} / \mathrm{Mn} / \mathrm{Ni} / \mathrm{Ti} / \mathrm{V}-001 / 12238
\end{aligned}
$$

$\mathrm{W}-074-\mathrm{Cr} / \mathrm{Mn} / \mathrm{Ni} / \mathrm{Ti} / \mathrm{V}-002 / 12239$

$\mathrm{W}-075-\mathrm{Ti} / \mathrm{V}-002 / 12240$

W-076-Poly-2/12236

W-077-Mo-001/12241

W-078-00-000/122.44

W-079-00-001/12248

$\mathrm{W}-080-\mathrm{Ph}-001 / 12254$

$\mathrm{W}-081-\mathrm{N} / \mathrm{Ni}-001 / 12256$

$\mathrm{i}-032-\mathrm{N} / \mathrm{V}-001 / 12258$

$\mathrm{i}-083-\mathrm{N} / \mathrm{Fe}-001 / 12260$

W-084-N/AI-001/12263

$\mathrm{W}-085-\mathrm{N} / \mathrm{Zr}-001 / 12265$

$W-080-E-001 / 12268$

$\mathrm{W}-087-\mathrm{Ca}-001 / 12269$

W*-08S-Cr-001/1H0524

$W^{*}-089-\mathrm{Cu}-001 / \mathrm{H} 0526$

$W^{*}-090-\mathrm{Mn}-001 / \mathrm{H}=0529$

$\mathrm{W}-091-\mathrm{Cr} / \mathrm{Mn}-002 / \mathrm{H}-0530$

$\mathrm{W}-092-\mathrm{Ph}-002 / \mathrm{H}-0533$

2.0
0.36
0.06
0.4
0.5

0.006

1.0

1.0

50

0.4

0.5

0.5

2.0

0.004

0.004

0.08

0.08

0.5

0.0006

0.0006

0.1

0.1

$$
\text { - } 0.1
$$

$-$

$--$

0.7

1.7

0.4

1

50

$<0.015$

400

?

0.5

2. 0

0.7

$0.5 / 0.3$

28
(2.0)

(0.75)

$(<0.2)$

$(0.3)$

$(0.7)$

$(<0.2)$

(1.0)

(<1.5)

(100)

$0.25(0.28)$

0.28

0.80

2.0

$<0.35$

$<0.35$

$<0.25$

$<0.25$

$<2.0$

$<0.25$

$<0.25$

$<0.25$

$<0.25$

$(<0.3)$

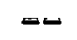

$-$

(0.7)

$(<2.0)$

$(0.85)$

(<1.5)

(40)

$(300500)$

$\dot{* *}$ Quantity in parenthesis is measured concentration.

*U.S. GOVERNMENT PRINTING OFFICE: 1977-740-306/265. Region 4. 